\title{
Theoretical systems of triboelectric nanogenerators
}

\author{
Simiao Niu ${ }^{1}$ and Zhong Lin Wang ${ }^{1,2, *}$ \\ ${ }^{1}$ School of Materials Science and Engineering, Georgia Institute of Technology, Atlanta, Georgia \\ 30332-0245, USA \\ ${ }^{2}$ Beijing Institute of Nanoenergy and Nanosystems, Chinese Academy of Sciences, Beijing, \\ 100083, China \\ *e-mail: zhong.wang@mse.gatech.edu
}

\begin{abstract}
:
Triboelectric nanogenerator (TENG) technology based on contact electrification and electrostatic induction is an emerging new mechanical energy harvesting technology with numerous advantages. The current area power density of TENGs has reached $313 \mathrm{~W} / \mathrm{m}^{2}$ and their volume energy density has reached $490 \mathrm{~kW} / \mathrm{m}^{3}$. In this review, we systematically analyzed the theoretical system of triboelectric nanogenerators (TENGs). Starting from the physics of TENGs, we thoroughly discussed the fundamental working principle of TENGs and simulation method. Then the intrinsic output characteristics, load characteristics, and optimization strategy is in-depth discussed. TENGs have inherent capacitive behavior and their governing equation is their $V-Q-x$ relationship. There are two capacitance formed between the tribo-charged dielectric surface and the two metal electrodes, respectively. The ratio of these two capacitances changes with the position of this dielectric surface, inducing electrons to transfer between the metal electrodes under SC condition. This is the core working mechanism of triboelectric generators and different TENG fundamental modes can be classified based on the changing behavior of these two capacitances. Their first-order lumped-parameter equivalent circuit model is a voltage source in series with a capacitor. Their resistive load characteristics have a "three-working-region" behavior because of the impedance match mechanism. Besides, when TENGs are utilized to charge a capacitor with a bridge rectifier in multiple motion cycles, it is equivalent to utilizing a constant DC voltage source with an internal resistance to charge. The optimization techniques for all TENG fundamental modes are also discussed in detail. The theoretical system reviewed in this work provides a
\end{abstract}


theoretical basis of TENGs and can be utilized as a guideline for TENG designers to continue improving TENG output performance.

Keywords:

Mechanical energy harvesting; triboelectric nanogenerator; $V-Q-x$ relationship; electrostatics;

\section{Introduction}

The tremendous development of portable electronics and sensor networks makes it an urgent requirement to develop sustainable and stable energy sources for them. Powering them entirely by batteries has become more and more unpractical and unfavorable, mainly for the limited battery lifetime, large scope of distribution of these kinds of electronic devices, and potential health and environmental hazards. Therefore, new technologies that can harvest energy from ambient environment as sustainable power sources are emerging research fields, called nano-energy research, which focuses on the applications of nanomaterials and nanotechnology for harvesting energy for powering micro/nanosystems. Recently, triboelectric nanogenerators (TENGs) based on contact electrification and electrostatic induction have become a promising technology in mechanical energy harvesting, which shows unique merits including large output power, high efficiency, low weight and cost effective materials, and simple fabrication.[1] Through 3 years research, the area power density of TENGs has reached $313 \mathrm{~W} / \mathrm{m}^{2}$ and their volume energy density has reached $490 \mathrm{~kW} / \mathrm{m}^{3}$.[2] The current developed highest mechanical energy conversion efficiency has reached about 85\%.[3] Triboelectric nanogenerators have been utilized as direct power source to charge a mobile phone battery [4] and worked as self-powered active sensors.[5] However, there are still requirements for continuing improving their output performance for more and more 
practical applications, which demands rational design and careful optimization of both materials and structures especially when the current TENG performance is already very high. Moreover, similar to the development of CMOS integrated circuits and systems, the fully-integrated energy harvesting systems that contain TENGs, power management circuits, signal processing circuits, energy storage elements, and/or load circuits are essential for the practical applications of the TENGs. Theoretical simulation plays a key role in understanding the working mechanism and analyzing the output performance of the entire system. Finally, from methodology point of view, simulation is always a necessary step in the whole device design process, because performing control experiments is usually time-consuming and not cost-effective. Thus a thorough theoretical understanding of TENGs is completely urgent in the whole research field. This knowledge can help choosing the appropriate TENG structure and materials, avoid designs which will greatly harm the output performance, and choose suitable systemlevel topologies for integrated energy harvesting systems.

The objective of this paper is to give a summary about the fundamental theory research of the triboelectric nanogenerator (TENG). First, we will discuss the governing equation, equivalent circuit model, and simulation method. Then, the basic output characteristics (open-circuit voltage, short-circuit transferred charges, and inherent capacitance) of four fundamental TENG modes are discussed in detail. Moreover, resistive load and capacitive load characteristics are shown. Finally, based on the above basic information, we will discuss some advanced TENG structures and optimization techniques for each fundamental TENG modes. 


\section{Basics of triboelectric nanogenerators}

\subsection{Inherent capacitive behavior and governing equations: $V-Q-x$ relationship}

The fundamental working principle of TENGs is a conjugation of contact electrification and electrostatic induction. Contact electrification provides static polarized charges and electrostatic induction is the main mechanism that converts mechanical energy to electricity. Since the most fundamental device based on electrostatics is a capacitor, fundamentally TENG will have inherent capacitive behavior.[6]

An arbitrary TENG is analyzed to unveil its inherent capacitive behavior. For any triboelectric generators, there are pair of materials which are face to each other (called tribo-pairs). The distance $(x)$ between these two triboelectric layers can be varied under the agitation of mechanical force. After being forced to get in contact with each other, the contact surface of the two triboelectric layers will have opposite static charges (tribocharges), as a result of contact electrification. Besides the tribo-pairs layer, there are two electrodes that are carefully insulated inside the TENG system, which ensures the charges can only transfer between the two electrodes through external circuits. If we define the transferred charges from one electrode to another is $Q$, one electrode will have the transferred charge $-Q$ and the other electrode will have the transferred charge $+Q$.

The electrical potential difference between the two electrodes of any TENGs mainly contributes to two parts. The first part is from the polarized triboelectric charges and their contribution to the voltage is $V_{\mathrm{OC}}(x)$, which is a function of separation distance $x$. Besides, the already transferred charges $Q$ will also contribute to an electric potential difference. If we assume no triboelectric charges exist in this structure, this structure is completely a typical capacitor, so the contribution of these already transferred charges between the two 
electrodes is $-Q / C(x)$, where $C$ is the capacitance between the two electrodes. Therefore, due to the electrical potential superposition principle, the total voltage difference between the two electrodes can be given by:[6]

$$
V=-\frac{1}{C(x)} Q+V_{\mathrm{OC}}(x)
$$

Equation 1 (named as $V-Q-x$ relationship) is the governing equation of any TENGs, clearly explaining its inherent capacitive behavior.[6] The separation of the polarized tribo-charges will generate an electrical potential difference between the two electrodes. If the external circuit exists between the two electrodes, this electrical potential will drive electrons to flow from one electrode to another. These already-transferred electrons can further screen the electrical potential between the two electrodes.[7] Under short-circuit conditions, these transferred charges $\left(Q_{\mathrm{SC}}\right)$ fully screen the electrical potential generated from polarized triboelectric charges. Therefore, the following equation can be easily derived for TENGs under short-circuit conditions.[8]

$$
0=-\frac{1}{C(x)} Q_{\mathrm{SC}}(x)+V_{\mathrm{OC}}(x)
$$

Thus, the fundamental relationship among $Q_{\mathrm{sc}}, C$, and $V_{\mathrm{oc}}$ can be given by:[8]

$$
Q_{\mathrm{SC}}(x)=C(x) V_{\mathrm{OC}}(x)
$$

\subsection{First-order lumped-parameter equivalent circuit model}

From the governing equation ( $V-Q-x$ relationship) of triboelectric nanogenerators, we can easily derive their lumped parameter equivalent circuit model.[9] From Equation 1 shown above, there are two terms at the right side, which can be represented by two circuit elements in the equivalent circuit model. The first one is a capacitance term, which is

originated from the inherent capacitance between the two electrodes and can be 
represented by a capacitor $(C)$. The other is an open-circuit voltage term, which is originated from the separation of the tribo-charges and can be represented by an ideal voltage source $\left(V_{\mathrm{OC}}\right)$. In combination of these two, the whole lumped parameter equivalent circuit model can be represented by a serial connection of an ideal voltage source and a capacitor, as shown in the next Figure.[9]

Besides, it's valuable to note that the inherent impedance of TENG mainly comes from its inherent capacitance. For a rationally designed TENG, the internal resistance is close to infinity mainly for the excellent insulation between the two electrodes. So in the firstorder equivalent circuit model, this resistance was not included.

Finally, we want to briefly discuss the comparison of TENG with traditional electromagnetic induction generators. First, their fundamental operating principles are different: TENGs rely on the conjugation effect of contact electrification and electrostatic induction while electromagnetic induction generators rely on the electromagnetic induction. Second, TENGs are inherently capacitive and the internal resistance for rational designed TENGs is close to infinity. Their internal high impedance is mainly from their small inherent capacitance. However, electromagnetic induction generators are inherently resistive and their internal resistance is much lower than the impedance of TENGs. Third, the equivalent circuit model for TENGs is a serial connection of an ideal voltage source with a capacitor while that for electromagnetic induction generators is a serial connection of an ideal voltage source with a resistor. In summary, TENGs are highimpedance charge sources and electromagnetic generators are low-resistance voltage sources.

\subsection{Theoretical simulation method}


The simulation of TENG-based energy harvesting systems mainly contains two parts, including electrostatic simulation and load circuit simulation. [9]

The main objective of the electrostatic simulation is to obtain the $V_{\mathrm{oc}}(x)$ and $C(x)$ functions in the $V-Q-x$ relationship. Two methods are currently developed to specify the $V_{\mathrm{OC}}(x)$ and $C(x)$ relationships. Analytical derivation is a preferred method but it only works for certain geometry features such as parallel-plate attached-electrode contactmode and sliding-mode TENGs with appropriate approximations. A more general method which works for all TENGs is numerical calculation based on finite element method (FEM). The finite element calculation can be easily performed utilizing common FEM simulation software such as COMSOL and ANSYS. In this numerical method, the value of $V_{\mathrm{OC}}$ and $C$ is first obtained at certain values of $x$. Then, from continuous fraction interpolation,[10] a numerical $V_{\mathrm{OC}}(x)$ and $C(x)$ relationship can be generated in the entire $x$ region.

Once the numerical $V_{\mathrm{OC}}(x)$ and $C(x)$ relationship is obtained, the output characteristics can be numerically calculated through load circuit simulation. When the load is very simple, the governing differential equation of whole system can be easily derived through Kirchhoff's law. For example, when the load is purely resistive, the governing differential equation containing the TENG and the resistor can be given by:[9]

$$
R \frac{\mathrm{d} Q}{\mathrm{~d} t}=V=-\frac{1}{C} Q+V_{\mathrm{OC}}
$$

Such a differential equation can be numerically solved by numerical integration and for some specific TENG system and motion condition, even analytical results can also be obtained. However, this kind of differential equation can only be obtained for limited 
cases with very simple load circuits. When the load circuit is complicated, solving the differential equation set is nearly an impossible task.

A general method that works for all TENG systems is to utilize SPICE software.[9] From the above TENG equivalent circuit model, the TENG can be embedded into the SPICE software as a basic element consisting of a voltage source in serial connection with a capacitor. Then any other circuit elements, including resistors, capacitors, inductors, diodes, and transistors can be put into the software to form the entire TENG system. After specifying the motion process and the initial condition, the powerful SPICE software can be easily utilized to simulate the real-time output of any TENG systems.

\section{Basic TENG output characteristics and fundamental modes}

Above we have discussed the basic knowledge and simulation method of triboelectric generators. In this chapter, we will move one-step further. From the basic electrodynamics, we will classify the TENGs by different modes from their fundamental electrostatic induction process. And for each TENG mode, we will discuss their basic output characteristics (open-circuit voltage $V_{\mathrm{OC}}$, short-circuit transferred charges $Q_{\mathrm{SC}}$, inherent capacitance $C$ ) in detail.

\subsection{Attached-electrode contact-mode triboelectric generators}

The first fundamental TENG working mode is called attached-electrode contact mode TENG, which is based on vertical charge separation mechanism.[7] This is also the earliest experimentally demonstrated TENG mode.[1, 11, 12] Based on the materials for the triboelectric pairs, the attached-electrode contact-mode TENG is mainly divided into two categories: dielectric-to-dielectric and conductor-to-dielectric types. The model built 
for a dielectric-to-dielectric contact-mode TENG is shown in Fig. 2a.[7] The two dielectric plates, with thicknesses of $d_{1}$ and $d_{2}$ and the relative dielectric constants $\varepsilon_{\mathrm{r} 1}$ and $\varepsilon_{\mathrm{r} 2}$, respectively, are stacked face to face as two triboelectric layers. At the outer surface of these two dielectrics, two metal layers are deposited as two electrodes. The distance $(x)$ between the two triboelectric layers can be varied under the agitation of mechanical force. After being forced to get in contact with each other, the inner surfaces of the two triboelectric layers will have opposite static charges (tribo-charges) with equal density of $\sigma$, as a result of contact electrification. For insulators as discussed, it is reasonable to assume that the tribo-charges are uniformly distributed at the two surfaces with negligible decay.[13, 14] When the two triboelectric layers start to separate from each other, with increased $x$, a potential difference $(V)$ between the two electrodes will be induced. The amount of transferred charges between the two electrodes, as driven by the induced potential, is defined as $Q$. We choose the minimum achievable charge reference state (MACRS),[15] so the instantaneous amount of charges on the electrode is $Q$ and $-Q$, respectively.

Similarly, the model for conductor-to-dielectric type is built, as shown in Fig. 2b. In this structure, we no longer have the Dielectric 1 and now the metal 1 plays two roles: both as the top triboelectric layer and as the top electrode. Therefore, the total charges in the metal 1 now have two parts: one is the triboelectric charges $(S \cdot \sigma)$, the other is the transferred charges between the two electrodes $(-Q)$. Thus, the total charges in the metal 1 are $(S \sigma-Q)$.

Since the area size $(S)$ of the metals is several orders of magnitude larger than their separation distance $\left(d_{1}+d_{2}+x\right)$ in the experimental case, it is reasonable to assume that 
the two electrodes can be seen as infinitely large. With the above model and the above assumption, the $V-Q-x$ relationship and basic output characteristics $\left(V_{\mathrm{OC}}, Q_{\mathrm{SC}}\right.$, and $C$ ) of such a contact-mode TENG can be derived based on electrodynamics, as shown below.[7]

$V=-\frac{Q}{S \varepsilon_{0}}\left(d_{0}+x(t)\right)+\frac{\sigma x(t)}{\varepsilon_{0}}$

$V_{O C}=\frac{\sigma x(t)}{\varepsilon_{0}} \quad(6 a) \quad Q_{S C}=\frac{S \sigma x(t)}{d_{0}+x(t)} \quad(6 b) \quad C=\frac{\varepsilon_{0} S}{d_{0}+x(t)}$

In the above equations, the effective dielectric thickness $d_{0}$ is defined as the summation of all the thickness of the dielectric $d_{i}$ between the two metal electrodes divided by its relative effective thickness $\varepsilon_{r i}$, as shown below.

$$
d_{0}=\sum_{i=1}^{n} \frac{d_{i}}{\varepsilon_{r i}}
$$

Besides utilizing the Gauss Theorem, we can derive its $V-Q-x$ relationship by the detailed electrostatic induction mechanism, through which we can show the fundamental working principle of such triboelectric generators.

We first need to define the nodes in this electrostatic system. A node in an electrostatic system corresponds to a surface/volume with the same electrical potential. Since the area size is assumed to be infinitely large and all the dielectric is uniform, the tribo-charged dielectric 2 surface is with the same electrical potential and can be thought as one node (Node 2). Since the electrical potential of the whole metal electrode is also the same, the electrode 1 and electrode 2 are other two nodes in this electrostatic system (Node 1 and Node 3, respectively), as shown in Figure $2 \mathrm{~b}$. Since every two nodes are connected by electric field lines, an equivalent capacitance is formed between each of them. However, since dielectric 2 surface is assumed to be infinitely large, the electrical line connection 
of Node 1 and Node 3 is fully blocked by the Node 2. Now only two capacitances exist in this electrostatic system, as shown in Figure 2c.

Now we consider this system under short-circuit condition, when the Node 1 and Node 3 has the same electrical potential. Under this condition, the total amount of charges on Node 1 and Node 3 is $\sigma S$ while that on Node 2 is $-\sigma S$. From charge conservation and Kirchhoff's Law, the amount of charge on Node 3 is easily given by:

$$
Q_{\# 3}=\frac{\sigma S}{1+\frac{C_{1}(x)}{C_{2}(x)}}
$$

So when $x$ is changing from 0 , the short circuit transferred charges from Node 3 to Node $1\left(Q_{\mathrm{SC}}\right)$ can be given by:

$$
Q_{\mathrm{SC}}=\frac{\sigma S}{1+\frac{C_{1}(x)}{C_{2}(x)}}-\frac{\sigma S}{1+\frac{C_{1}(x=0)}{C_{2}(x=0)}}
$$

Utilizing the parallel-plate capacitance mode, Equation 9 can be easily derived to be the same with Equation 6b. Through the above derivation, it is clearly observed that the core working mechanism of TENGs is that the change of the capacitance ratio $C_{1} / C_{2}$ due to the change of the position of tribo-charged surface will induce electron transfer between the electrodes under SC condition.

When the $x$ is sufficiently large, the charge transfer efficiency $\eta_{\mathrm{CT}}$ (defined as the ratio between the final transferred charges and the total triboelectric charges) can be given by:

$$
\eta_{\mathrm{CT}}=\frac{Q_{\mathrm{SC}, \mathrm{final}}}{\sigma S}=\frac{1}{1+\frac{C_{1}\left(x=x_{\max }\right)}{C_{2}\left(x=x_{\max }\right)}}-\frac{1}{1+\frac{C_{1}(x=0)}{C_{2}(x=0)}}
$$


For the above attached-electrode contact-mode TENGs, when $x=0, C_{1}\left(\varepsilon_{0} S / x\right)$ is close to infinity while $C_{2}$ is a finite number $\varepsilon_{0} S / d_{0}$, which ensures the amount of charge on Node 3 at this time is 0 . When $x$ is sufficiently large (larger than $\left.10 d_{0}\right), C_{1}\left(\varepsilon_{0} S / x\right)$ is close to 0 while $C_{2}$ is still $\varepsilon_{0} S / d_{0}$, which ensures the amount of charge on Node 3 at this time is $\sigma S$. Therefore, the maximum charge transfer efficiency for an attached-electrode contactmode TENG can be reach $100 \%$.

From the above derivation, the basic output characteristics of attached-electrode contactmode TENGs have the following properties. Their open-circuit voltage is linearly proportional to the separation distance $x$ while their inherent capacitance is inversely proportional to the separation distance $x$. For short-circuit transferred charges, it has a saturation trend. $Q_{\mathrm{SC}}$ quickly reaches $90 \%$ of the final saturation value $\sigma S$ when $x$ only changes from 0 to $10 d_{0}$. (Shown in Figure 4 with a comparison to single-electrode TENGs) Since the dielectric thickness is usually very small, the minimum separation distance must be effectively reduced to 0 to reach high charge transfer efficiency. If the minimum separation distance is comparable to $10 d_{0}, C_{1}(x)\left(\varepsilon_{0} S / x\right)$ will always be much smaller than $C_{2}(x)\left(\varepsilon_{0} S / d_{0}\right)$. Under this condition, the capacitance ratio $C_{1}(x) / C_{2}(x)$ will always be close to 0 and changes little when $x$ further increases, which will lead to no charge transfer between the electrodes. Besides, when $x$ is already comparable to the size

of the device, the device can be no longer seen as infinitely large and edge effect will dominate the performance. Under this condition, the open-circuit voltage will deviate from the linear relationship.

\subsection{Attached-electrode sliding-mode triboelectric generators}


The second fundamental working mode is called attached-electrode sliding-mode triboelectric generators, which is based on in-plane charge separation mechanism.[6] Several previous experimental works also have also been demonstrated.[5, 16-19] Considering the materials to be used as the pair of the triboelectric layers, the attachedelectrode sliding-mode TENG also has two types: dielectric-to-dielectric and conductorto-dielectric. We first take the dielectric-to-dielectric category as an example. The model for this type of TENG was built with dimensions close to those of the real device, as shown in Figure 3a. Considering that the width $w$ of the whole structure is much larger than the thickness, a 2D model was utilized to simplify the calculation. The geometric size of the two dielectrics was $l$ in longitudinal direction and $d_{\mathrm{i}}(i=1,2)$ in thickness direction. Two metal electrodes were bonded with the two dielectrics layers. The bottom part was fixed while the top could slide through the longitudinal direction. The lateral separation distance was defined as $x$. When the two dielectrics were separated, the lower surface of Dielectric 1 and the upper surface of Dielectric 2 had charges with different signs at the non-overlapped regions, which are because of the triboelectric effect. Same as attached-electrode contact-mode TENGs, it is reasonable to assume that those triboelectric charges (tribo-charges) are uniformly distributed on these two surfaces, which is an excellent approximation for insulators. Also, the decay of tribo-charges with time can be neglected on these insulating polymers. The overall density of tribo-charges on the overlapped surface can be regarded as 0 because the distance between the center of the positive charges and the negative charges are in atomic level. The surface density of the tribo-charges is defined as $\sigma$ at surface of Dielectric 1 and $-\sigma$ at surface of Dielectric 2 . Under MACRS and OC condition, the total charge at each metal electrode was assigned 
as 0 . If the transferred charges amount is defined by $Q$, the total charge amount on metal 1 and metal 2 is $-Q$ and $Q$, respectively. For conductor-to-dielectric attached-electrode sliding-mode TENGs, the only geometrical structure difference is the absence of Dielectric 1. In this case, Metal 1 is not only the top triboelectric layer, but also the top electrode itself. When Metal 1 and Dielectric 2 are separated, due to the triboelectric effect, the upper surface at the separated region of Dielectric 2 has the uniform tribocharges (surface density is $-\sigma$ ) and the same amount of tribocharges with opposite sign will exist on the surface of Metal 1. Therefore, at the OC condition (MACRS), the total amount of charges on the bottom electrode is 0 . For the ease of physical understanding, the tribo-charges on the dielectric surface in the overlapped region has been deemed to cancel the opposite tribo-charge portions on the top metal layer, with the density of $\sigma$ and amount of $\sigma w(l-x)$. Thus, in our model, the total amount of charges on the top electrode is $\sigma w x$.

The analytical equation for the general case of attached-electrode sliding-mode TENGs cannot be derived and rigorous theoretical analysis can only base on the numerical method. But in the practical applications, since $l$ is always much larger than $d_{1}$ and $d_{2}$ and $x$ is always smaller than $0.9 l$ (because of the difficulty of perfect alignment of the two dielectric surfaces), an approximate analytical $V-Q-x$ relationship can be derived when the edge effect can be neglected under the above conditions.

To derive the $V-Q-x$ relationship, the analytical estimation of $C(x)$ and $V_{\mathrm{OC}}(x)$ must first be obtained. We take the dielectric-to-dielectric case as an example. The capacitance $C$ is first deducted. Since the thickness of the dielectrics is much smaller than their length, the capacitor between the overlapped region is the dominate part of the total capacitance as 
long as the two dielectrics are not close to thoroughly separated. Therefore, utilizing the parallel-plate capacitor model, the total capacitance $C$ can be estimated by the following equation:

$$
C=\frac{\varepsilon_{0} w(l-x)}{d_{0}}
$$

where $d_{0}$ is the effective dielectric thickness as defined in equation 7 .

$V_{O C}$ can be estimated by understanding the charge distribution. Since the length of the dielectrics is much larger than the thickness, we can assume in each region, the metal electrodes work as infinite plates. Under this approximation, the charge distribution is uniform in each region, and the electric field is uniform along the Y-direction inside the dielectrics. (This conclusion can also be verified through FEM calculation results[6]). Therefore, in an ideal case, the absolute value of surface charge density is $\sigma$ for the nonoverlapped region. For the overlapped region, the charge density is still uniform. Thus, the charge density at the overlapped region can be calculated through the 0 total charges at each electrode under OC conditions. The ideal charge distribution under OC condition (utilizing MACRS) can be approximated by the following equations:

For the non-overlapped region of the bottom electrode: $\rho=\sigma$

For the overlapped region of the bottom electrode: $\rho=-\frac{\sigma x}{l-x}$

For the non-overlapped region of the top electrode: $\rho=-\sigma$

For the overlapped region of the top electrode: $\rho=\frac{\sigma x}{l-x}$ 
Utilizing the charge distribution shown above and Gauss Theorem, $V_{\mathrm{OC}}$ can be easily estimated as:

$V_{O C}=\frac{\sigma x d_{0}}{\varepsilon_{0}(l-x)}$

So the $V-Q-x$ relationship for attached-electrode sliding-mode TENGs when the edge effect can be neglected can be shown as:

$$
V=-\frac{1}{C} Q+V_{O C}=-\frac{d_{0}}{w \varepsilon_{0}(l-x)} Q+\frac{\sigma d_{0} x}{\varepsilon_{0}(l-x)}
$$

For conductor-to-dielectric TENGs when the edge effect can be neglected, similar analysis shows the same results.

The electrostatic induction process of attached-electrode sliding-mode TENGs can also be thoroughly understood through the node discussion similar to the attached-electrode contact-mode TENGs. For simplicity, we take the conductor-to-dielectric attachedelectrode sliding-mode TENGs as an example mainly because it only has one tribocharged surface and we only analyze the capacitance distribution at the two ends ( $x=0$ and $x=x_{\max }>l$ ). When $x=0$, the capacitance between the tribo-charged dielectric surface and the top electrode is infinity while the capacitance between the tribo-charged dielectric surface and the bottom electrode is a finite large number, so the ratio between these two capacitances is infinity. When a full separation has already been reached, the capacitance between the tribo-charged dielectric surface and the top electrode is nearly 0 while the capacitance between the tribo-charged dielectric surface and the bottom electrode maintains still same, so the ratio between these two capacitances is now 0 . The change of 
capacitance ratio due to the change of the relative position of tribo-charged surface will induce electron transfer between the electrodes under SC condition. From the above discussion, the detailed working mechanism of attached-electrode sliding-mode TENGs is almost the same as that of attached-electrode contact-mode TENGs and the maximum charge transfer efficiency for this kind of TENG can also reach $100 \%$.

From the above derivation, the basic output characteristics of attached-electrode slidingmode TENGs have the following properties. Their short-circuit transferred charge is linearly proportional to the separation distance $x$ while their inherent capacitance is linearly proportional to the length of the overlapped region $(l-x)$. For open-circuit voltage, it increases dramatically when $x$ is close to $l$ due to the quick decrease of the capacitance.

One important consideration for attached-electrode sliding-mode TENGs is that their output of is very sensitive to the gap between the two tribo-pair surfaces. For example, if the thickness of such gap is the same as $d_{0}$, the capacitance between the tribo-charged dielectric surface and the top electrode when $x=0$ is the same with the other capacitance. And the capacitance ratio can only change from 1 to 0 when $x$ changes from 0 to $x_{\max }$. Therefore, the charge transfer efficiency will be degraded to only $50 \%$, as shown in Figure $3 \mathrm{c}$. Since $d_{0}$ is always a small number, the gap tolerance of attached-electrode sliding-mode TENGs is poor, which can be improved by utilizing sliding-mode freestanding TENGs described in the following section.

Besides, another important consideration for attached-electrode sliding-mode TENGs is the edge effect.[6] When the thickness of the dielectrics is comparable to their length, strong edge effect will dominate the output and the charge transfer efficiency will be 
strongly degraded. Figure $3 \mathrm{~d}$ shows the FEM simulation results for a conductor-todielectric attached-electrode TENG whose dielectric constant is 2 . When the aspect ratio of length and dielectric thickness reduces from 500 to 0.05 , the charge transfer efficiency reduces from nearly $100 \%$ to about $14 \%$. Usually the edge effect can be ignored in practical sliding-mode TENGs with only one unit. However, this effect will become dominant in grating-structured TENGs when the grating is fine enough, which we will discuss in detail in the following section.

\subsection{Single-electrode triboelectric generators}

Traditional attached-electrode contact-mode or sliding-mode TENGs require one electrode and the corresponding lead to be attached to the moving part of the devices, which limits their applicability. To overcome this drawback, some new structure of TENGs that eliminates the moving electrode has been demonstrated, which include single-electrode TENGs (SETENGs) and freestanding-triboelectric-layer nanogenerators (FTENGs). In this section, we will discuss the fundamental working mechanism of SETENGs.[8] There are also many experimental demonstrations for SETENGs.[20, 21] In SETENGs, only one electrode is attached to (or serves as) the triboelectric layer (called primary electrode). The other electrode serves as an electric potential reference and can be placed anywhere in the space, even directly be the ground (called reference electrode). The function of the two electrodes in SETENGs is not equal to each other. Similar to attached-electrode triboelectric generators, SETENGs also have contact-mode and sliding-mode, which shows almost the same characteristics. Therefore, we mainly focus on the contact-mode SETENGs in the following discussions. 
We first take the conductor-to-dielectric contact-mode SETENGs as an example. Its finite element model was built in 2-Dimensional to simplify the numerical calculation without losing its inherent physics meaning, as shown in Figure 4a. One dielectric plate and one metal electrode (primary electrode) are stacked face to face to form the triboelectric pair, with the same length of $l$ and width of $w$. The thickness is $d_{0}$ for Dielectric 1 and $d_{m}$ for the primary electrode. As one typical configuration, the reference electrode of the same geometric size locates underneath the primary electrode, with a gap of $g$. The two electrodes are fixed and Dielectric 1 can move along the vertical direction under external mechanical force, with the distance between the two triboelectric layers defined as $x$. As a result of contact-electrification with the primary electrode, Dielectric 1 carries net negative charges with a density of $-\sigma$ on its bottom surface. Considering the excellent insulation properties of the polymer and the nature of contact electrification, it can be assumed that the charges are uniformly distributed on the surface at macro scale with negligible decay. At the same time, there will be equal amount $(\sigma w l)$ of positive charges injected to the primary electrode through contact electrification. With $Q$ defined as the transferred charges from the primary electrode to the reference electrode, the total amount of charges at the primary electrode and reference electrode under MACRS are $\sigma w l-Q$ and $Q$, respectively. [8]

The FEM calculation results of $C, V_{O C}$, and $Q_{S C}$ for the contact-mode SETENG are plotted in Figure 4b-d, in comparison with the corresponding results of the attachedelectrode contact-mode TENG with the same size. (Detailed calculation parameter is listed in Table 1). Their characteristics are completely different. First, due to the fixture of the SETENG's electrodes, its $C$ is nearly a constant $\left(C_{0}\right)$ with the increase of $x$. 
However, for the attached-electrode structure, its $C$ decreases quickly when the distance between the two electrodes is increasing. Secondly, in the SETENG, its $V_{O C}$ quickly gets saturated at a value much lower than that of the attached-electrode structure. This is because when Dielectric 1 is far away from the electrodes, the influence of Dielectric 1 on the electric field distribution around the electrodes is little. Therefore, further increase in $x$ will contribute little to the increase of $V_{O C}$. Finally, in the SETENG, $Q_{S C}$ slowly reached its saturation value, which is only half of the value for the paired-electrode structured TENG. When Dielectric 1 is far away from the electrodes, the tribo-charges generated are equally distributed between the two electrodes to maintain the two electrodes at the same electric potential. The maximized $\eta_{C T}$ can only reach $50 \%$, while $\eta_{C T}$ for the attached-electrode TENGs can reach nearly $100 \%$ even when $x$ is relatively small.

The degradation of output performance of SETENGs mainly comes from the electrostatic shield effect of the primary electrode, which can be analyzed from their equivalent circuit model.[8] The nodes are still first analyzed in the electrostatic system. For the above system in OC condition, the electric potential across the whole bottom surface of Dielectric 1 is nearly constant, as shown in the FEM calculation results. Thus, the whole surface can be assumed as a node (Node 1). Similarly, the primary electrode and the reference electrode also serve as two nodes, which can be named as Node 2 and Node 3, respectively. Since every two nodes are connected by electric field lines, an equivalent capacitance is formed between each of them, as shown in Figure 4e. Their capacitances $C_{1}, C_{2}$, and $C_{3}$ in the equivalent circuit only represent the capacitive effects from the direct electric line connection between every two nodes without crossing any charged 
objects (without electrostatic shield of the third obeject), so they are not actual capacitances that reflects all the electric line connection (both direct and non-direct) between the two nodes and can be directly measured.

As a quantitative relationship between the two categories of capacitance, the actual capacitance is the combination of these three capacitances. For example, the actual capacitance $\left(C_{b}\right)$ between Node 1 and Node 3 contains two parts: $C_{2}$ and the serial connection of $C_{1}$ and $C_{3}$ that shows the capacitance effect of non-direct electric lines connection through Node 2, as given by:

$C_{b}=C_{2}+\frac{C_{1} C_{3}}{C_{1}+C_{3}}$

Similarly, the actual capacitances between Node 1 and Node $2\left(C_{a}\right)$ and between Node 2 and Node $3\left(C_{0}\right)$ can also be given as below:

$C_{a}=C_{1}+\frac{C_{2} C_{3}}{C_{2}+C_{3}}$

$C_{0}=C_{3}+\frac{C_{1} C_{2}}{C_{1}+C_{2}}$

At OC condition (MACRS), the total charges on Node 1, 2, and 3 are $-\sigma w l, \sigma w l$, and 0 , respectively. Thus, from basic characteristics of capacitances and charge conservation on each node, $V_{\mathrm{OC}}, Q_{S C}$ and $\eta_{C T}$ can be given by $\left(C_{1}(x=0)\right.$ is close to infinity in the following equation):[8]

$Q_{S C}=\frac{\sigma w l}{1+\frac{C_{1}(x)}{C_{2}(x)}}-\frac{\sigma w l}{1+\frac{C_{1}(x=0)}{C_{2}(x=0)}}=\frac{\sigma w l}{1+\frac{C_{1}(x)}{C_{2}(x)}}$ 
$\eta_{C T}=\frac{Q_{S C}}{\sigma w l}=\frac{1}{1+\frac{C_{1}(x)}{C_{2}(x)}}-\frac{1}{1+\frac{C_{1}(x=0)}{C_{2}(x=0)}}=\frac{1}{1+\frac{C_{1}(x)}{C_{2}(x)}}$

$V_{O C}=\frac{\sigma w l C_{2}}{C_{1} C_{2}+C_{2} C_{3}+C_{3} C_{1}}$

Equation 18 and 19 are the same as the above equation in the attached-electrode case, which shows that the core electrostatic induction principle of SETENGs is the same with attached-electrode TENGs. However, in SETENGs, the trend of the capacitance is different from the attached-electrode TENG case. When $x=0, C_{a}$ goes to infinity while $C_{b}$ and $C_{0}$ become equal with each other. Therefore, from Equation $8-10, C_{1}$ goes to infinity while $C_{2}$ and $C_{3}$ are equal to $C_{0} / 2$, which is a finite number. Therefore, the ratio $C_{1} / C_{2}$ is infinity. When $x$ approaches to infinity, both $C_{a}$ and $C_{b}$ approach to 0 at the same rate. Therefore, both $C_{1}$ and $C_{2}$ approach to 0 with the ratio of $C_{1} / C_{2}$ approaching to 1 . Thus, under this condition, the transferred charges is only half amount of the tribocharges and the maximum charge transfer efficiency is only $50 \%$.

From Equation 18-20, we observe that the capacitance $C_{2}$ is critically important for both $V_{O C}$ and $Q_{S C} . C_{2}$ represents the portion of the electric lines directly connecting Node 1 and Node 3, without being electrostatically shielded by Node 2. If all the electric lines connections between the reference electrode and Dielectric 1 needs to come through the primary electrode (electrostatic shielded by the primary electrode), $C_{2}$ will be equal to 0 , which will lead to no output from the SETENG. Therefore, SETENG with infinite area size will lead to zero output due to the fully electrostatic shiled condition.

\section{4 freestanding-triboelectric-layer based triboelectric generators}


The output performance of SETENGs is limited by the electrostatic shield effect of their primary electrodes and the maximum charge transfer efficiency can only reach 50\%.[8] To overcome this drawback, another kind of fixed-electrode TENGs are designed with the tribo-charged surface alternatively approaching the two electrodes, which is the freestanding-triboelectric-layer based triboelectric generators (FTENGs).[15] Same as SETENGs, the moving object of an FTENG does not need to be connected to the TENG system as well.[3, 4, 22, 23] FTENGs can also be utilized to harvest mechanical energy from any moving object. [22]However, unlike the SETENGs, FTENGs don't have this electrostatic shield effect problem. Besides, the output characteristics of contact-mode FTENGs (CFTENGs) and sliding-mode FTENGs (SFTENGs) are completely different and we will discuss their characteristics separately.

\subsection{1 contact-mode freestanding-triboelectric-layer based triboelectric generators}

Two typical structures of CFTENGs are shown in Figure 5. [15]Figure 5a shows a typical structure for dielectric-freestanding-layer CFTENGs. A dielectric plate (thickness: $d_{1}$, relative dielectric constant: $\varepsilon_{\mathrm{r} 1}$ ) and two metal plates are stacked face to face, forming two triboelectric pairs. The two metal plates also serve as two electrodes. The total air gap thickness between these two metal plates is defined as $g$. After the dielectric plate being forced to contact with the two metal plates, both the top and the bottom surfaces of Dielectric 1 will have static triboelectric charges (tribo-charge) due to contact electrification. For simplicity, we assume the triboelectric charge density of both surfaces is the same $(-\sigma)$. At the same time, the two metal plates will have the same amount of positive charges in total because of charge conservation. 
The above system can be easily solved through Gauss's Law. But to fully show its inherent physics and working behavior, we still try to utilize the concept of nodes to analyze this electrostatic system. In practical applications, the size of the metal electrode is always much larger than the air gap. Therefore, the area size of the freestanding contact-mode TENG $(S)$ is seen as infinitely large and the edge effect can be ignored. As a result, the electric potential on metal 1 , metal 2 , the top and bottom surface of dielectric 1 are all constant, so these 4 surfaces can be seen as 4 nodes in this electrostatic system. The electrical line connection between every two nodes forms an equivalent capacitance between them. Since the area size $(S)$ is assumed as infinitely large, the electrical line connection between every two non-adjacent nodes (for example, Node $1 \& 3$ ) is fully blocked by the intermediate node (for example, Node 2). Therefore, only three capacitances exist in the equivalent circuit model of this electrostatic system, as shown in Figure 5b. The total capacitance between the two electrodes (Node $1 \& 4$ ) is the serial connection of $C_{1}, C_{2}$, and $C_{3}$, which can be easily given by the following equation.

$$
C=\frac{1}{\frac{1}{C_{1}}+\frac{1}{C_{2}}+\frac{1}{C_{3}}}=\frac{\varepsilon_{0} S}{d_{0}+g}
$$

Inside equation 21 , the definition of the effective dielectric thickness $d_{0}$ is the same with the previous definition.

The charge distribution under short circuit (SC) condition when Node 1 and Node 4 have the same electrical potential is then analyzed. The charges on each node need to be deducted to solve this electrostatic system. For both Node 2 and 3, the amount of charges on the node is $-\sigma S$. In addition, because of charge conservation, we only know that the 
total charges on Node 1 and 4 are $2 \sigma S$, but the detailed charge distribution is still unknown. Under SC condition, we set the total charges on metal 1 (Node 1) is $Q_{1}$ and the total charges on metal 2 (Node 4$)$ is $Q_{2}\left(Q_{2}=2 \sigma S-Q_{1}\right)$. From the basic electrodynamics theory and charge conservation, the total charges on metal 1 and 2 can be easily solved as:

$$
Q_{1}=\sigma S \frac{\frac{1}{C_{2}}+\frac{2}{C_{3}}}{\frac{1}{C_{1}}+\frac{1}{C_{2}}+\frac{1}{C_{3}}}(22 a) \quad Q_{2}=\sigma S \frac{\frac{2}{C_{1}}+\frac{1}{C_{2}}}{\frac{1}{C_{1}}+\frac{1}{C_{2}}+\frac{1}{C_{3}}}(22 b)
$$

In practical applications, the effective dielectric thickness of the dielectrics $d_{1} / \varepsilon_{\mathrm{r} 1}$ is always negligible compared to the air gap and $C_{2}$ can be seen as infinitely large. Thus, equation (22a) and 22) can be further simplified to:

$$
Q_{1} \approx \sigma S \frac{\frac{2}{C_{3}}}{\frac{1}{C_{1}}+\frac{1}{C_{3}}}=\frac{2 \sigma S}{1+\frac{C_{3}}{C_{1}}}(23 a) \quad Q_{2} \approx \frac{2 \sigma S}{1+\frac{C_{1}}{C_{3}}}(23 b)
$$

From Equation 23a and 23b, the basic working mechanism of CFTENGs can be easily observed. When $x=0, C_{3}$ is infinity, as a result, $Q_{1}$ is close to 0 and $Q_{2}$ is approximately $2 \sigma S$. Under this condition, all the positive tribo-charges are attracted to the bottom electrode by the negative charges on the surface of dielectric 1 . While when $x=g, C_{1}$ is infinity and all the positive charges on the electrodes are attracted to Metal 1 . Therefore, if Dielectric 1 is under a vibration inside the air gap, charges will alternatively flow between Metal 1 and Metal 2 due to the change of $C_{1} / C_{3}$, forming an $\mathrm{AC}$ short circuit current. Same as all previous TENGs, the change of the capacitance ratio between the tribo-charged surfaces and the two electrodes induced by the change of Dielectric 1 position can drive electrons to flow under SC conditions between the two electrodes. 
Under MACRS, the short circuit transferred charges $\left(Q_{\mathrm{SC}}\right)$ and open-circuit voltage can be easily obtained as:

$$
\begin{gathered}
Q_{S C}=Q_{1}(x)-Q_{1}(x=0)=\frac{2 \sigma S x}{d_{0}+g} \\
V_{O C}=\frac{2 \sigma x}{\varepsilon_{0}}
\end{gathered}
$$

Therefore, the governing equation for this CFTENG, which is its $V-Q-x$ relationship, can be shown as:[15]

$$
V=-\frac{1}{C} Q+V_{O C}=-\frac{d_{0}+g}{\varepsilon_{0} S} Q+\frac{2 \sigma x}{\varepsilon_{0}}
$$

Besides the dielectric-freestanding-layer CFTENGs, another important category is metalfreestanding-layer CFTENGs, as shown in Figure 5c. The working principle of metalfreestanding-layer CFTENGs is very similar to the dielectric-freestanding-layer case. Similar to the previous discussed attached-electrode contact-mode TENGs, metal can be simply seen as dielectrics with 0 thickness to calculate $d_{0}$ and the derived equations above are all applicable to metal CFTENGs.

With the above information, the comparison of the basic output characteristics of CFTENGs and attached-electrode contact-mode TENGs can be provided. Their $V_{\mathrm{OC}}$ characteristics are similar to each other, which are both linearly proportional to $x$. However, their inherent capacitance and short-circuit transferred charges characteristics are completely different. First, the inherent capacitance of CFTENGs is constant and independent of $x$ while that of contact-mode attached-electrode TENGs decreases when $x$ 
increases. In addition, $Q_{\mathrm{SC}}$ of FETENGs is also linearly proportional to $x$ while that of attached-electrode TENGs has a saturation trend.

\subsection{2 sliding-mode freestanding-triboelectric-layer based triboelectric generators}

Besides CFTENGs, another important category is the sliding-mode FTENGs (SFTENGs) based on in-plane charge separation mechanism.[15] Based on the freestanding material, SFTENGs have two subcategories, dielectric SFTENGs whose freestanding materials only contain dielectrics and metal SFTENGs whose freestanding materials contains metal. The basic model of these two SFTENGs is shown in Fig. 6a and b. In the SFTENGs, Metal 1 and 2 are placed in the same plane with a gap $g$ to form two electrodes. A freestanding layer with the same size of Metal 1 stands on the top of the metal electrodes and the distance between the bottom surface of freestanding layer and Metal 1 is defined as the freestanding height $h$. The width of this structure is defined as $w$. For dielectric SFTENGs shown in Fig. 6a, the freestanding layer only contains a layer of dielectrics. And for metal SFTENGs, a thin layer of metal is deposited at the backside of the dielectric freestanding layer. These two structures look very similar, but unlike CFTENGs, the freestanding material will strongly influence the output characteristics of the SFTENGs.

The main working principle of SFTENGs is very similar to that of CFTENGs. The node concept can still be utilized to analyze their working principle. We first analyze dielectric SFTENGs. In contrast to CFTENGs, since the electrical potential of the dielectric bottom surface is not a constant, this surface could not be seen as a single node. The electric potential superposition principle is utilized to solve this problem. First, we assume that 
only a small region of $\mathrm{d} k$ in the bottom dielectric surface (the distance of this region to the left edge of the bottom dielectric surface is $k$ ) contains the tribo-charges with a density of $-\sigma$, and correspondingly the total charges on metal 1 and 2 are $\sigma w \mathrm{~d} k$. Utilizing the similar derivation method we utilized above, the total charges on metal 1 and 2 ( $\mathrm{d} Q_{1}$ and $\mathrm{d} Q_{2}$ ) under $\mathrm{SC}$ conditions because of these tribo-charges can be given by the following equations, where $C_{\mathrm{i}}(k)$ states for the capacitance between this small surface $\sigma w \mathrm{~d} k$ and metal i:

$$
\mathrm{d} Q_{1}=\frac{\sigma w \mathrm{~d} k}{1+\frac{C_{2}(k)}{C_{1}(k)}}(26 a) ; \quad \mathrm{d} Q_{2}=\frac{\sigma w \mathrm{~d} k}{1+\frac{C_{1}(k)}{C_{2}(k)}}(26 b)
$$

Because of the superposition principle of the electrostatic field, the total charges on metal 1 and metal 2 considering the whole charges on the dielectric surface is the integration of each small tribo-charged region, which can be shown as:

$$
Q_{1}=\sigma w \int_{0}^{l} \frac{\mathrm{d} k}{1+\frac{C_{2}(k)}{C_{1}(k)}}(27 a) ; \quad Q_{2}=\sigma w \int_{0}^{l} \frac{\mathrm{d} k}{1+\frac{C_{1}(k)}{C_{2}(k)}}
$$

Therefore, $Q_{\mathrm{SC}, \text { final }}$ can be shown as:

$$
Q_{\mathrm{SC}, \mathrm{final}}=\int_{0}^{l} \frac{\sigma w \mathrm{~d} k}{1+\left(\frac{C_{2}(k)}{C_{1}(k)}\right)_{x=g+l}}-\int_{0}^{l} \frac{\sigma w \mathrm{~d} k}{1+\left(\frac{C_{2}(k)}{C_{1}(k)}\right)_{x=0}}
$$

When $x=0$, the tribo-charged dielectric surface is much closer to metal 1 than to metal 2, so the ratio $C_{2}(k) / C_{1}(k)$ is close to 0 for all the $k$ values. Therefore, $Q_{1}$ will be approximately $\sigma w l$ and $Q_{2}$ will be approximately 0 from equation 27 . On the contrast, the 
ratio $C_{2}(k) / C_{1}(k)$ is close to infinity for all the $k$ values when $x=g+l$. Therefore, $Q_{1}$ will be approximately 0 and $Q_{2}$ will be approximately $\sigma w l$. Thus, $Q_{\mathrm{SC}, \text { final }}$ can reach $\sigma w l$ and the charge-transfer efficiency $\left(\eta_{\mathrm{CT}}\right)$ defined as the ratio between $Q_{\mathrm{SC}, \text { final }}$ and the total tribo-charges can reach $100 \%$. Same as CFTENGs, the change of the ratio of these two capacitances with the change of $x$ serves as the driving force for electrons to transfer between the two electrodes.

For metal SFTENGs, the basic electrostatic induction principle is the same as the dielectric SFTENGs. Therefore, as shown in Fig. 6c, the numerically calculated trend of $Q_{S C}$ profile (under MACRS) is quite similar to that of dielectric SFTENGs. (Detailed numerical calculation parameter is listed in Table 2). However, unlike CFTENGs, their characteristics of capacitance and $V_{\mathrm{OC}}$ are completely different from each other, as shown in the numerical results presented in Fig. 6d and 6e. The capacitance profile of dielectric SFTENGs is nearly constant when the position of the freestanding layer changes. However, for metal SFTENGs, the capacitance when the freestanding layer is in the middle is several orders larger than that when the freestanding layer is at the two ends. This capacitance difference leads to the difference of their $V_{\mathrm{OC}}$ profile. Since the capacitance of metal-SFTENGs in the middle region is much larger than that of dielectric-SFTENGs, so from equation 3, the open-circuit voltage of metal-SFTENGs in this region will be much smaller than that of dielectric-SFTENGs. And when $x$ is close to $g+l$, since the capacitance of the metal-SFTENG quickly dropped to the value close to the dielectric-SFTENG, the open-circuit voltage of the metal-SFTENG also quickly increase to a value close to the dielectric-SFTENG. Therefore, although the final open-circuit voltage values of the two SFTENGs are almost the same, the metal-SFTENG curve is 
much more non-linear than the dielectric-SFTENG curve and the maximum slope of the metal-SFTENG curve is much larger than that of the dielectric-SFTENG curve.

These differences in capacitance and $V_{\mathrm{OC}}$ profiles are mainly from the charge redistribution inside the metal freestanding layer. In dielectric-SFTENGs, the charges are not free to move. Since the relative dielectric constant of the commonly utilized dielectrics is not much larger than the relative dielectric constant of the air (always in the range of 2-4), the change of the capacitances due to the change of the freestanding-layer position is little. However, the charge on the surface of metal freestanding layer can redistribute to keep the electrical potential of the metal freestanding layer constant, which will lead to huge disturbance of the electrostatic field distribution. Therefore, the capacitance of metal-SFTENGs has two parts. The first part is the direct parasitic capacitance between these two electrodes, which can be analogous to the capacitance of dielectric SFTENGs. The second part is the serial connection of the capacitance between metal 1 and metal $3\left(C_{\mathrm{f} 1}\right)$ and metal 3 and metal $2\left(C_{\mathrm{f} 2}\right)$, which is the unique capacitance part of metal SFTENGs. When both the dielectric thickness and the freestanding height $h$ are not too large, the contribution of this serial connection is very significant to the total capacitance. At the two ends, either $C_{\mathrm{f} 1}$ or $C_{\mathrm{f} 2}$ will close to 0 because the metal freestanding layer only have overlapping region with one electrode, so the total capacitance of metal SFTENGs is close to the capacitance of dielectric SFTENGs. When $x$ equals to $(g+l) / 2, C_{\mathrm{f} 1}$ and $C_{\mathrm{f} 2}$ will be the same and their serial connection gets its maximum, leading to the largest total capacitance under this condition. At the same time, strong charge redistribution has been observed in the inner surface of the metal freestanding layer. 


\subsection{Summary of the TENG fundamental modes}

In summary, the fundamental working mechanism of TENGs is a conjugation of contact electrification and electrostatic induction. Through contact electrification, electrostatic charges can be generated and kept on the dielectric surface. There are two equivalent capacitances will form between the tribo-charged dielectric surface and the two metal electrodes, respectively. The change of the ratio of these two capacitances due to the change of the position of the dielectric will induce electrons to transfer between the metal electrodes under SC condition. The difference of the capacitances' changing behavior gives rise to the different fundamental modes of TENGs. There are 4 fundamental modes in TENGs, whose capacitance changing trend is summarized in Table 3.

\section{Resistive Load Characteristics}

\subsection{Resistive Load Characteristics and "three-working-region" behavior}

Resistive load is the simplest load case for TENGs. When TENGs are connected with a resistive load $R$, the equivalent circuit of the whole system is shown in Figure 7a. Utilizing Kirchhoff's law, the governing equation of the whole system can be easily given by: $[6,7]$

$$
R \frac{\mathrm{d} Q}{\mathrm{~d} t}=-\frac{1}{C} Q+V_{O C}
$$

The above equation is a first-order differential equation and can be easily solved by specifying the initial boundary condition $Q(t=0)$. There are two types of boundary conditions that are most commonly utilized. First, we can consider a very common case that the device has stopped at the equilibrium position $(x=0)$ for a long time and 
electrostatic equilibrium has been reached before the motion process. If $V_{\mathrm{OC}}$ are utilized its value under MACRS, the boundary condition is $Q(t=0)=0$. Second, if the TENG is under a periodic motion with a specific period, output from any initial boundary condition will gradually converge to a periodic output wave after the first few periods, which is the steady-state. To solve the steady-state solution, periodic boundary condition must be utilized, which is $Q(t=0)=Q(t=\mathrm{T})$, where $\mathrm{T}$ is the period of the motion process. For simplicity, in this section, we mainly utilized the boundary condition $Q(t=$ $0)=0$, which will not affect the main conclusion. Under this boundary condition, equation 11 can be solved as:

$$
Q(t)=\frac{1}{R} \exp \left[-\frac{1}{R} \int_{0}^{t} \frac{1}{C(x(t))} d t\right] \int_{0}^{t} V_{O C}(x(t)) \exp \left[\frac{1}{R} \int_{0}^{t} \frac{1}{C(x(t))} d t\right] d t
$$

$I(t)$

$$
\begin{aligned}
& =\frac{V_{O C}}{R}-\frac{1}{R^{2} C} \exp \left[-\frac{1}{R} \int_{0}^{t} \frac{1}{C(x(t))} d t\right] \int_{0}^{t} V_{O C}(x(t)) \exp \left[\frac{1}{R} \int_{0}^{t} \frac{1}{C(x(t))} d t\right] d t(30 b) \\
& V(t) \\
& =V_{O C}-\frac{1}{R C} \exp \left[-\frac{1}{R} \int_{0}^{t} \frac{1}{C(x(t))} d t\right] \int_{0}^{t} V_{O C}(x(t)) \exp \left[\frac{1}{R} \int_{0}^{t} \frac{1}{C(x(t))} d t\right] d t(30 c)
\end{aligned}
$$

The above equations are a bit complicated. As a typical example, we consider an attached-electrode contact-mode TENG under a constant velocity motion process.[7] The motion process is shown as the following equation and the parameters are listed in Table 4.

$$
x=v t\left(t<\frac{x_{\max }}{v}\right) \quad(31 a) \quad x=x_{\max }\left(t \geq \frac{x_{\max }}{v}\right)
$$

The numerical calculated results are shown in figure $7 \mathrm{~b}-\mathrm{f}$. The detailed profiles of the charge, current, and voltage relationships at different loads are shown in Fig. 7b-d. At SC 
condition, the charge transfers at the fastest speed. For a relatively small $R, Q$ can still get its saturation value when the top electrode stops moving $(t=10 \mathrm{~ms})$. However, when $R$ is more than $100 \mathrm{M} \Omega$, at $t=10 \mathrm{~ms}$, the charge cannot get saturated due to the limit of the charge transfer rate by the resistor, resulting in the unstopped charge transfer from metal 1 to metal 2 after $t=10 \mathrm{~ms}$. Therefore, the current is a peak-shape when $R$ is small while the current continues increasing during the plate movement when $R$ is large. The voltage has the same profile with the current, but a different trend in magnitude. The peak values of the voltage, current and power on different loads are displayed in Fig. 7e and f. It can be clearly observed that the operation of the TENG can be divided into three working regions. First, In Region I where the resistance is low (0.1-1000 $\Omega$ ), the peak current has little drop from the SC condition, which is due to the similar charge transfer process in comparison with that of the SC condition. As a result, the maximum voltage is approximately proportional to the external resistance. On the other hand, when the resistor is larger than $1 \mathrm{G} \Omega$ (Region III), the output characteristics are close to the OC condition, in which the maximum voltage saturates at $V_{O C}$. The medium range of the resistance is Region II, where the maximum current drops dramatically but the maximum voltage increases at a reverse trend. Around $100 \mathrm{M} \Omega$ inside this region, the TENG reaches its maximum instantaneous output power.

This unique output characteristic of TENGs can be interpreted both physically and mathematically.[7] In physics, we can understand this unique characteristic from two different views. As an intuitive sense, we can explain this three-working-region behavior from the charge transfer rate point of view. The working principle of the TENG is a conjunction of the triboelectric process and the electrostatic induction. When the tribo- 
charges are separated, an induced voltage will be generated between the two electrodes, which will drive the electrons to flow from electrode 2 to electrode 1 . The electric field from these transferred charges will screen the original electric field from the tribocharges. The charge accumulation rate at SC condition is the maximum charge transfer rate, which is determined by the mechanical motion. However, as there is an external load with certain resistance, the resistor will limit the real charge transfer rate, making it lower than that at the SC condition. When the load resistance is very small, this limitation is not obvious so that the rate can still catch up with the maximum rate determined by the moving mode, enabling the $Q$ to stay close to $Q_{S C}$ in Region I. When the load resistance continues to increase and gets into Region II, it begins to significantly limit the charge flow rate, making the charge accumulation curve deviate downward from $Q_{S C}$. As the load resistance is large enough, the transfer of electrons from metal 2 to metal 1 is rather slow, which results in a very small current. The induced voltage between the two electrodes will hold for a long time due to the little screening effect, with the magnitude approaching $V_{O C}$. This is the Region III of the TENG.

Besides the explanation from charge transfer rate, this unique three-working-region behavior can also be understood from the impedance match between the inherent TENG capacitance and the load resistance, although now there is no strict definition of the impedance of the inherent TENG capacitance because the TENG capacitance is timevariant and the $V_{\mathrm{OC}}$ is also not a pure harmonic function. When the load resistance is small, its impedance is much smaller than the impedance of the TENG capacitance. Thus, the total impedance is dominated by the TENG inherent capacitance and the output current remains almost unchanged when the resistance increases. When the load 
resistance is large enough, its impedance is much larger than the impedance of the TENG capacitance. Thus, almost all of the $V_{\mathrm{OC}}$ is applied on the load resistance and the TENG is working under a quasi-OC condition. When the load resistance is in the middle region (matches the TENG impedance), the maximum transit power can be reached.

Mathematically, this can be interpreted by considering the two limitation conditions. When $R$ approaches 0 , equation 30a can be easily simplified to be $C V_{\mathrm{OC}}$, which is just the same equation with $Q_{S C}$. The TENG is now in the quasi-SC condition (Region I). When $R$ approaches infinity, equation $30 \mathrm{c}$ can be easily simplified to be $V_{\mathrm{OC}}$, which is just the open-circuit voltage. The TENG is now in the quasi-OC condition, which is the Region III. When $R$ is neither too large nor too small, the behavior of the TENG is in the transitional region between SC and OC conditions (Region II), within which the maximum power is reached.

\subsection{Optimum Resistance}

In this three-working-region behavior of the TENG, the optimum resistance for the maximum instantaneous power is a key parameter to maximize the effective efficiency of the TENG. Therefore, the position of the optimum resistance needs to be systematically studied to find its relationship with the TENG's structural parameters and operational conditions, so that the TENG can be rationally controlled for different application purposes.

From the impedance match process we discussed above, the influence of structural parameters and motion condition on the optimum resistance can be easily addressed. For a TENG, its average impedance is approximately $1 /\left(\omega C_{\text {avg }}\right)$, where $\omega$ is the angular 
frequency of the signal source $V_{\mathrm{OC}}$ and $C_{\text {avg }}$ is the average TENG inherent capacitance. Any structural parameters that can increase $C_{\text {avg }}$ will lower the TENG impedance and lower the optimum resistance. Increasing the motion velocity is equivalent to the increase of $\omega$, which will also lower the optimum resistance.

As a typical example, we can analyze the optimum resistance of an attached-electrode contact-mode TENG. Through rigorous mathematical derivation, the optimum resistance $\left(R_{\mathrm{opt}}\right)$ of this attached-electrode contact-mode TENG under any arbitrary smooth motion process can be given by:[7]

$$
R_{o p t}=\frac{d_{0}{ }^{2}}{\left[H\left(\frac{x_{\max }}{d_{0}}\right)\right]^{2} S v \varepsilon_{0}}
$$

In the above equation, $H$ is a function that only relies on the detailed moving process and all the other parameters has the same meaning as listed in Table 4. This equation can be easily understood from the fundamental impedance match process. An increase in the area size of the TENG will increase the TENG capacitance than will lower its impedance. The optimum resistance reduces as a response. Similarly, increasing average velocity is equivalent to increase the high frequency component of $V_{\mathrm{OC}}$, which will reduce the TENG impedance as well. As a result, the optimum resistance will decrease. If the effective dielectric thickness increases, the inherent TENG capacitance will reduce and its impedance will increase, so the optimum resistance increases. In addition, increasing the gap will reduce the average capacitance and increase the average impedance, so the optimum resistance also increases. From the similar analysis, since the TENG impedance is independent of the tribo-charge density, the optimum resistance will not be affected if the tribo-charge density changes. 
These conclusions of optimum resistance can also be validated through numerical calculation and experimental studies.[7] As shown in Fig. 8a, $R_{\text {opt }}$ is inversely proportional to either the area size $(S)$ or the average velocity $(v)$. On the other hand, the influences of effective dielectric thickness and air gap distance on $R_{\mathrm{opt}}$ are displayed in Fig. 8b. If we keep the ratio (y) of the above two thicknesses $\left(d_{0}\right.$ and $\left.x_{\max }\right)$ as constant of 27.2, $R_{\mathrm{opt}}$ is proportional to $d_{0}^{2}$. Two set of experiments were also performed to validate the theoretical results. In the first set of experiments, the same device was measured under different average velocities, as shown in Fig. 8c. When the average velocities are $0.02 \mathrm{~m} / \mathrm{s}, 0.04 \mathrm{~m} / \mathrm{s}$, and $0.08 \mathrm{~m} / \mathrm{s}$, the derived optimum load resistance (through interpolation of the experiment data) is $14.2 \mathrm{M} \Omega, 6.66 \mathrm{M} \Omega$, and 4.08 $\mathrm{M} \Omega$, respectively, which shows the same trend with the theoretical estimation. In the other set of measurements, the influence of the gap and the dielectric thickness is experimentally studied. The thickness of the Kapton film $\left(d_{k}\right)$ in device 1 is $25 \mu \mathrm{m}$ while that of device 2 is $125 \mu \mathrm{m}$, and the corresponding gaps $\left(x_{\max }\right)$ are $2 \mathrm{~mm}$ and $10 \mathrm{~mm}$, respectively, which maintains the same $y$. Through the measurement on a series of resistors with all the other parameters kept the same, the obtained optimum resistance for device 1 is $7.69 \mathrm{M} \Omega$ while that of device 2 is $162.3 \mathrm{M} \Omega$. The experimental optimum resistance ratio is 21.1 , which is quite close to 25 , as the theoretical estimation value.

\section{Capacitive load and charging characteristics}

In the above chapter the resistive load characteristics of TENG are discussed. In this chapter, we will discuss another important load condition: capacitive load. [24]We will start from the simplest unidirectional motion charging characteristics. Then a much more 
complicated multiple-cycle charging process was analyzed to show the unique TENG charging characteristics and an optimum load capacitance was observed for maximized energy storage. To obtain the optimized design strategy for such system, the analytical solution of the optimum load capacitance was deducted and its dependence on the charging cycle numbers and TENG structural parameters was shown.

\subsection{TENG charging performance under unidirectional motion condition}

We start our discussion with the simplest case, which is utilizing a single-electrode contact-mode TENG to charge a load capacitor $\left(C_{\mathrm{L}}\right)$ under a unidirectional mechanical motion. In this process, no external rectifier is needed because the charging current of the TENG is also unidirectional. Besides, the inherent capacitance $\left(C_{\mathrm{T}}\right)$ of a single-electrode TENG is nearly constant, which will also simplify our discussion. The model of the single-electrode contact-mode TENG is the same as we described above in Section 3.3. Utilizing the above equivalent circuit model of TENG, the equivalent circuit model of this whole system is shown as Fig. 9a. In the discussion, $Q$ was defined as the transferred charges from the primary electrode to the reference electrode and $Q^{\mathrm{C}}$ was defined as the charges on the top plate of the load capacitor.

To solve the above equivalent circuit, the initial condition must be specified. As a typical example, we consider the case that at $t=0$, the top dielectric has stopped at $x=0$ position for a long time and just starts moving. Besides, no initial charges are stored on the load capacitor, so initial charges on both $C_{\mathrm{T}}$ and $C_{\mathrm{L}}$ are $0\left(Q(t=0)=Q^{\mathrm{C}}(t=0)=0\right)$. Thus, the following equations can be obtained from Kirchhoff's Law and node charge conservation, where $V$ stands for the voltage across $C_{\mathrm{L}}$.

$$
V=-\frac{1}{C_{T}} Q+V_{\mathrm{OC}}=\frac{1}{C_{L}} Q^{C}
$$




$$
Q^{C}-Q=Q^{C}(t=0)-Q(t=0)=0
$$

In practical application, the motion of the dielectric always has a maximum separation distance, which is defined as $x_{\max }$. Thus, the final voltage and charge on the capacitor when a full separation is reached ( $x$ reaches $x_{\max }$ ) can be obtained by solving the above two equations. ( $V_{\mathrm{OC}, \max }$ and $Q_{\mathrm{SC}, \max }$ stands for the open circuit (OC) voltage and short circuit (SC) transferred charge of the TENG when $x=x_{\max }$ )

$$
\begin{gathered}
V\left(x=x_{\max }\right)=\frac{C_{T} V_{\mathrm{OC}, \max }}{C_{L}+C_{T}}=\frac{Q_{\mathrm{SC}, \max }}{C_{L}+C_{T}} \\
Q^{C}\left(x=x_{\text {max }}\right)=\frac{C_{L} Q_{\mathrm{SC}, \max }}{C_{L}+C_{T}}
\end{gathered}
$$

Thus, the total energy stored in the capacitor $\left(E^{\mathrm{C}}\right)$ can be given by:

$$
E^{C}=\frac{1}{2} C_{L}\left[V\left(x=x_{\max }\right)\right]^{2}=\frac{C_{L}\left[Q_{\mathrm{SC}}\left(x=x_{\max }\right)\right]^{2}}{2\left(C_{L}+C_{T}\right)^{2}}
$$

To obtain an intuitive sense of such equations, numerical calculation for the singleelectrode TENG under constant velocity motion condition is performed and the final voltage and charge storage amount under different load capacitances are shown in Figure 9b-c. (The detailed calculation parameters are the same as listed in Table 1 and now the value of $x_{\max }$ is $2 \mathrm{~cm}$ ). When $C_{\mathrm{L}}$ is small, its impedance is much larger than the impedance of $C_{\mathrm{T}}$. Thus, almost all of the $V_{\mathrm{OC}}$ is applied on $C_{\mathrm{L}}$ and the TENG is working under a quasi-OC condition. However, the stored charge is still close to 0 because of the small $C_{\mathrm{L}}$, leading to a limited final stored energy. On the opposite, when $C_{\mathrm{L}}$ is very large, its impedance is much smaller than the impedance of $C_{\mathrm{T}}$, so the voltage applied on $C_{\mathrm{L}}$ is approximately 0 . At this moment, the TENG is working under a quasi-SC condition. However, the total stored energy is still limited because of the low voltage across $C_{\mathrm{L}}$. 
From the above analysis, only at the transition region the stored energy can reach its maximum. Mathematically, it can be easily derived from equation 37 that the optimum load capacitance $\left(C_{\mathrm{L}, \mathrm{opt}}\right)$ at which the maximum stored energy is reached is equal to $C_{\mathrm{T}}$, as shown in Figure 9c. Physically, this means the impedance match between the TENG and the load is reached.

\subsection{TENG charging performance under periodic mechanical motion}

Above we analyzed the charging behavior of a single-electrode TENG to charge a load capacitor under a unidirectional motion. However, in practical applications, the mechanical motion is always not unidirectional, resulting in an AC charging current. Therefore, a full-bridge diode rectifier is necessary to prevent the charge leaking back from $C_{\mathrm{L}}$ to the TENG. Besides, the inherent capacitance of most TENGs is time-variant. Therefore, the case in real applications is much more complicated than the above discussion. The equivalent circuit model of a practical TENG charging circuit is shown in Fig. 10a. As a typical example, the case we analyzed in the following section is a contact-mode attached-electrode conductor-to-dielectric TENG under a periodic harmonic motion process (shown in (38)).

$$
x=x_{\max }\left[\frac{1}{2}-\frac{1}{2} \cos \left(\frac{\pi v}{x_{\max }} t\right)\right]
$$

The structure of this TENG is just shown in Figure 2b. Utilizing the parameters listed in Table 5, we can obtain the numerical results of this non-linear time-variant system shown in Figure 10a from the TENG-simulator. The simulation results are plotted in Figure 10bd. As shown in Figure 10b, a saturation charging curve is observed for all the load 
capacitors, which is very similar to a typical RC charging curve in shape. At $t=0$, the load capacitor is charging at a maximum speed. Then the charging speed gradually slows down and finally the same saturation voltage about $104.8 \mathrm{~V}$ is reached for all the different load capacitors. It takes less time to charge a smaller $C_{\mathrm{L}}$ to reach its saturation voltage. However, a different trend is observed for the stored charges. At beginning, the curves for all load capacitors converge to one linear curve, with the charging rate of $2 Q_{\mathrm{SC} \text {,max }}$ per cycle. However, when time increases, the curves from small $C_{\mathrm{L}}$ shift downwards first and finally get saturated. At this moment, very few charges can be charged into the capacitor. The final voltage and the stored charge of the load capacitor after 1000 charging cycles has the similar trend as in the unidirectional charging case shown in Figure $9 \mathrm{~b}$. When $C_{\mathrm{L}}$ is small enough, the voltage on the load capacitor is approximately its saturation voltage while the stored charges are close to 0 and proportional to $C_{\mathrm{L}}$. When $C_{\mathrm{L}}$ is large enough, the stored charge is approximately $2 k Q_{\mathrm{SC} \text {,max }}$ while the voltage is close to 0 and inversely proportional to $C_{\mathrm{L}}$. Similar to the previous unidirectional charging, an optimum capacitance for maximum stored energy is also observed in the transition region of $C_{\mathrm{L}}$.

In addition to the demonstrated numerical calculation, an analytical solution is preferred to better understand the underline physics despite the fact that only approximate solution can be derived. Through linearization of the diodes and neglect of the diode conducting voltage, the voltage on $C_{\mathrm{L}}$ at the end of $k$ th charging cycle $\left(\left|V_{k, 2 \mathrm{end}}^{\mathrm{C}}\right|\right)$ can be derived as:[24]

$$
\left|V_{k, \text { end }}^{C}\right|=\frac{Q_{S C, \text { max }}}{C_{\min }+C_{\max }}\left\{1-\left[1-\frac{2\left(C_{\min }+C_{\max }\right) C_{L}}{\left(C_{L}+C_{\max }\right)\left(C_{L}+C_{\min }\right)}\right]^{k}\right\}
$$


In the above equation, $C_{\max }$ and $C_{\min }$ stand for the value of $C_{\mathrm{T}}(x=0)$ and $C_{\mathrm{T}}\left(x=x_{\max }\right)$, respectively. $Q_{\mathrm{SC}, \max }$ stands for the value of $Q_{\mathrm{SC}}\left(x=x_{\mathrm{max}}\right)$. In practical applications, $C_{\mathrm{L}}$ is usually much larger than both $C_{\max }$ and $C_{\min }$. Under this condition, equation 39 can be further simplified to: (where $f$ stands for the frequency of the periodic motion)

$$
\left|V_{k, 2 \mathrm{end}}^{C}\right|=\frac{Q_{S C, \max }}{C_{\min }+C_{\max }}\left\{1-\exp \left[-\frac{2\left(C_{\min }+C_{\max }\right) f t}{C_{L}}\right]\right\}
$$

Equation 40 clearly shows that a TENG is completely comparable to a DC voltage source in series with a resistor regarding the charging characteristics. They all follow the same exponential saturation trend. The value of the DC source is the saturation voltage $\left(V_{\text {sat }}\right)$ shown in Figure xa and its value can be easily calculated as:

$$
V_{\text {sat }}=\lim _{k \rightarrow \infty}\left|V_{k, \text { end }}^{C}\right|=\frac{Q_{S C, \max }}{C_{\min }+C_{\max }}
$$

Same as a first order RC charging circuit, $V_{\text {sat }}$ is only a function of TENG parameters but is independent of $C_{\mathrm{L}}$. Besides the saturation voltage, the charging speed is another important charging parameter. Analogous to a first-order $\mathrm{RC}$ charging circuit, time constant $(\tau)$ is utilized to characterize the charging speed, which can be easily given by:

$$
\tau=\frac{C_{L}}{2\left(C_{\min }+C_{\max }\right) f}
$$

The time constant is directly proportional to $C_{\mathrm{L}}$. Therefore, the value of the effective resistor in the first-order RC charging circuit can be easily extracted as $1 /\left[2 f\left(C_{\max }+C_{\min }\right)\right]$, which shows that the TENG with a larger inherent capacitance can charge $C_{\mathrm{L}}$ with a higher speed. 
Moreover, when $C_{\mathrm{L}}$ is large enough, linear charging behavior is observed and the TENG is working on quasi-SC condition. When $C_{\mathrm{L}}$ is close to infinity, the final stored charges at the end of the $k$ th charging cycle can be given by:

$$
\begin{gathered}
\lim _{C_{\mathrm{L}} \rightarrow \infty}\left|V_{k, \text { end }}^{C}\right|=\lim _{C_{\mathrm{L}} \rightarrow \infty} \frac{2 k Q_{S C, \text { max }}}{C_{L}}=0 \\
\lim _{C_{\mathrm{L}} \rightarrow \infty}\left|Q_{k, \text { end }}^{C}\right|=C_{\mathrm{L}} \lim _{C_{\mathrm{L}} \rightarrow \infty}\left|V^{C}{ }_{k, \text { end }}\right|=2 k Q_{S C, \text { max }}
\end{gathered}
$$

Since the maximum charge generated from the TENG is $Q_{\mathrm{SC} \text { max }}$ for each half cycle, the above results show that all the charges generated from the TENG are stored in $C_{\mathrm{L}}$. At the same time, the voltage difference across the TENG is 0 , which is completely consistent with the numerical results shown in Figure 10b.

Finally and most importantly, there also exists an optimum load capacitance $\left(C_{\mathrm{L}, \mathrm{opt}}\right)$ at which the stored energy reaches its maximum value. This optimum load capacitance is critically important for optimized design for such energy harvesting system. Through mathematical derivation, the approximate solution of $C_{\mathrm{L}, \mathrm{opt}}$ can be obtained as: (valid when $k$ is larger than 10)

$$
C_{\mathrm{L}, \mathrm{opt}}=1.592 k\left(C_{\min }+C_{\max }\right)
$$

From the above derivation, some conclusion can be reached. First, $C_{\mathrm{L}, \mathrm{opt}}$ is linearly dependent on the charging cycle numbers $k$. Second, it is the summation of $C_{\min }$ and $C_{\max }$ that determines the charging characteristics of TENGs. In attached-electrode contactmode and sliding-mode TENGs, $C_{\min }$ is much smaller than $C_{\max }$ and only $C_{\max }$ has critical effect on the total charging characteristics. Third, the maximum value of $V_{\mathrm{oc}}$ (equals to $\left.Q_{\mathrm{sc}, \max } / C_{\min }\right)$ is not the direct parameter that determines the final saturation voltage, but $Q_{\mathrm{sc}, \max } / C_{\max }$. In attached-electrode contact-mode and sliding-mode TENGs with $x_{\max }$ large 
enough to reach a nearly $100 \%$ charge transfer efficiency, although continuing increasing $x_{\max }$ is still beneficial to generate a high open-circuit voltage due to the decrease of $C_{\min }$, this will not provide any help on the charging performance.

\section{Structure influence and optimization for improved TENG output performance}

In the above chapters, the basic output characteristics and basic load effects of triboelectric generators have been shown. In this chapter, we will discuss some in-depth output characteristics of different modes of triboelectric generators and the influence of several important structural parameters on the output performance. Based on this discussion, some optimization strategies are proposed to obtain an improved TENG output performance.

\subsection{Attached-electrode grating-structured TENGs and influence of the edge effect}

Grating structure is an advanced structural design based on the above discussed slidingmode TENGs.[5, 17, 19] Utilizing grating structure, the in-plane charge separation cycles can by multiplied. However, the output characteristics of grating structure are very complicated mainly because the edge effect can no longer be neglected. The length of one grating unit is usually comparable to the dielectric thickness when the grating is fine. In this section, in-depth theoretical models of two types of grating TENGs, one with equal length plates and the other with unequal length plates, are discussed in detail. [25] Through theoretical and computational methods, their output characteristics are obtained and analyzed. With this basic understanding of the output performance, the effect of finer 
pitches is outlined for both grating structures. In addition, structural optimization strategies are provided to maximize the power output.

\subsubsection{Grating Structure with Equal-length Plates}

\subsubsection{Influence of electrode structure}

The grating structure with two equal-length plates is first considered. In a grating TENG, the patterning of the triboelectric layer is essential in order to enable multiple charge separation cycles. On the other hand, patterning is not necessary for the attached electrodes. As shown in Fig. 11a, there are two choices available: a continuous plate electrode and a grating electrode, in which the electrodes are fabricated with the same grating as the dielectric layer. In this case, the electrical connection is realized through external circuits or electrode bars. These two electrode configurations will lead to different output performance.

To compare the output characteristics of the two grating structures with different electrode configurations, their finite element method (FEM) models were built and calculated using COMSOL. Since the width of grating TENGs is usually much larger than their thickness, 2D models were utilized to simplify the calculation. Two grating dielectrics with $50 \%$ duty cycle are placed as tribo-pairs (a pair of materials which will undergo contact electrification). Their half pitch is defined as $l$ and the number of grating units in the top plate is defined as $n$. The total length of the top plate is $L$, such that $L=$ 2nl. As an example, the case with two grating units $(n=2)$ was studied to illustrate the difference. (Detailed calculation parameters are listed in Table 6.) Due to contact electrification, different signs of static charges (called tribo-charges) are distributed at the lower surface of Dielectric 1 and the upper surface of Dielectric 2. We assume that the 
tribo-charges are uniformly distributed on these surfaces with a density of $\sigma$. In order to simulate the relative-sliding motion in operation, the bottom dielectric is fixed and the top dielectric slides in the lateral direction, with the lateral displacement defined as $x$.

The profiles of $V_{O C}$ and $Q_{S C}$ generated by these two TENGs (Under MACRS) with different electrode structures are completely different, as shown in Fig. 11b-d. $V_{O C}, Q_{S C}$, and the charge transfer efficiency $\eta_{C T}$ of both structures show an oscillating trend, but those of the plate electrodes are always smaller than those of the grating electrodes. If the AC output is rectified to DC signal for storage, the TENG with grating electrodes would contribute a higher amount of accumulated charge. $\eta_{C T \text {-rectified }}$ after a full displacement cycle from the grating electrode structure can reach $200 \%$ while that from the plate electrode only reaches $126 \%$. It should be noted that the reason that $\eta_{C T \text {-rectified }}$ is larger than $100 \%$ for both structures comes from the multiple cycles of charge separation, which is the key advantage of the grating structure. As for the open circuit voltage, $V_{O C}$ after a full displacement cycle from the grating electrode can reach around $31 \mathrm{kV}$ while that from the plate electrode only reaches around $10 \mathrm{kV}$. This set of comparisons clearly shows that the grating electrode is more effective in electricity generation than the plate electrode.

This difference in output characteristics results from the difference in charge distribution at the metal electrodes in these two electrode structures, as shown in Fig. 11e and $\mathrm{f}$ for the example case of $x=l$ under SC conditions. $Q_{s c}$ equals half of the difference between the total amount of charge on top electrodes and on the bottom electrodes, which can be regarded as the sum of the contributions in all sub-regions (as marked in Fig. 11e and f). 
As an example, we can analyse the charge distribution in Region II and its contribution to $Q_{s c}$ to elucidate the difference between these two electrode structures.

For the grating electrode, only the top electrode exists in this region. Therefore, the induced charge density on the top electrode is $\sigma$ and its contribution to $Q_{s c}$ is $\sigma w l / 2$. For the plate electrode, both the top and bottom electrode exist in this region. A charge density of $\sigma_{T}$ will be induced on the top electrode, and $\sigma_{B}$ on the bottom. Thus its contribution to $Q_{s c}$ is $\left(\sigma_{T}-\sigma_{B}\right) w l / 2$. Since the electric field inside the metal electrodes is 0 , the following relationship exists:

$\sigma_{T}+\sigma_{B}=\sigma$

In addition, because the voltage between the top and bottom electrodes is 0 under SC conditions, we will have:

$\frac{\sigma_{T}}{\varepsilon_{0}} \frac{d_{1}}{\varepsilon_{r 1}}=\frac{\sigma_{B}}{\varepsilon_{0}} d_{2}$

This equation indicates that $\sigma_{T}$ and $\sigma_{B}$ will have the same sign. From Equation $46,\left(\sigma_{T}-\sigma_{B}\right)$ will be obviously smaller than $\sigma$. Thus, the contribution of the plate electrode in region II to $Q_{s c}$ is smaller than that of the grating electrode structure. Likewise, in regions III and IV, their contribution to $Q_{s c}$ of the plate electrode is also smaller than that of the grating electrode. As a result, the total $Q_{S C}$ generated by the plate electrode will be smaller. Therefore, through comparison of plate and grating electrode structures, it can be concluded that grating electrode structure is favourable for high output power. Thus, in the following discussion, we will mainly focus on TENGs with grating electrodes.

\subsubsection{Influence of number of grating units $(n)$}

The number of grating units $(n)$ has a direct impact on the total output characteristics. When $n$ starts to increase from 1, the approximate ideal charge distribution is still 
satisfied because $l$ is still much larger than the thickness of dielectrics. However, when $n$ is increased to fairly large values and $l$ is comparable with $d_{1}$ or $d_{2}$, the non-ideal edge effect is significant and cannot be neglected any longer.

The influence of $n$ in relatively small regions which satisfies the ideal conditions is first discussed. In this case, the instantaneous $Q_{S C}$ and the short circuit current $\left(I_{S C}\right)$ have the following relationships with $n$ :

$$
\begin{gathered}
Q_{S C}=\frac{\sigma w L}{2}\left[\frac{k}{n}+\frac{2(n-k)}{L}(x-2 k l)\right], 2 k l \leq x \leq(2 k+1) l \quad(k \in N) \\
Q_{S C}=\frac{\sigma w L}{2}\left[1-\frac{2(n-k-1)}{L}(x-2 k l-l)\right],(2 k+1) l \leq x \\
\quad \leq(2 k+2) l(k \in N) \quad(48 \mathrm{~b}) \\
I_{S C}=\frac{\mathrm{d} Q_{S C}}{\mathrm{~d} x} \frac{\mathrm{d} x}{\mathrm{~d} t}=\sigma w(n-k) \frac{\mathrm{d} x}{\mathrm{~d} t}, 2 k l \leq x \leq(2 k+1) l \quad(k \in N) \\
I_{S C}=\frac{\mathrm{d} Q_{S C}}{\mathrm{~d} x} \frac{\mathrm{d} x}{\mathrm{~d} t}=-\sigma w(n-k-1) \frac{\mathrm{d} x}{\mathrm{~d} t},(2 k+1) l \leq x \leq(2 k+2) l \quad(k \in N)
\end{gathered}
$$

Therefore, the following equations give the accumulated charges $\left(Q_{S C \text {-rectified }}\right)$ and charge transfer efficiency $\left(\eta_{\text {CT-rectified }}\right)$ after rectification under SC conditions when a full displacement is finished:

$$
\begin{gathered}
Q_{S C, \text { rectified }}(x=L)=\frac{\sigma w L}{2} n \\
\eta_{C T, \text { rectified }}=\frac{Q_{S C, \text { rectified }}(x=L)}{Q_{\text {tribo-total }}}=n
\end{gathered}
$$

From the above derivation, the peak value of $I_{S C}$ and $Q_{S C \text {-rectified }}$ in the grating structure can be enhanced by $n$ times through the subdivision process under ideal conditions, which is the most significant advantage of fabricating finer pitch structures. 
To verify these results, a numerical calculation for a grating TENG under ideal conditions $(L / d=727.3$, detailed calculation parameters are the same as shown in Table 6) is performed and the results are shown in Fig. 12a-d. The numerically calculated results are consistent with the theoretical analysis above. When $n$ increases, the number of charge transfers increases as a response, which significantly elevates the amount of accumulated charges after rectification. In addition, the slope of $Q_{S C}-t$ curve also increases with $n$, leading to an enhancement of $I_{S C}$, as shown in Fig. 12b. But as for voltage, since finer pitch results in a significant increase in capacitance when the top dielectrics and bottom dielectrics are fully separated, the peak value of $V_{O C}$ at larger $n$ significantly drops while the peak value of $Q_{S C}$ remains almost the same, which is determined by the general $V_{O C}$, $Q_{S C}$ and $C$ equation (equation 3).

Such a change in $V_{O C}$ is shown in Fig. 12c. However, the total energy generated is more complicated, as it is determined by both the current and the voltage. Fig. 12d shows the energy generated by 3 TENG structures with different $n$ in one full back-and-forth cycle under different load resistances. The following conclusions can be reached by comparing the curves shown in Fig. 12d. First, a finer pitch can generate more energy in the low resistance range, where the energy is mainly dominated by $I_{S C}$. However, a wider pitch can generate more energy in the high resistance range, where the generated energy is mainly determined by $V_{O C}$. The optimum resistance (the resistance at which the total energy is maximized) decreases with $n$, due to the increase of the inherent TENG capacitance. Considering the peak value of the harvested energy (in Fig. 12d), a finer pitch doesn't significantly improve the output, as $V_{O C}$ decreases more significantly than $I_{S C}$ increases for the equal-length grating TENGs. 
When $n$ continues to increase to very large values, the aspect ratio of each unit will be further lowered and the non-ideal edge effect cannot be neglected. To study the influence of this non-ideal effect, a numerical calculation for grating TENGs with a small $L / d$ ratio of 16 was conducted, as shown in Fig. 12e and f. (Detailed calculation parameters are shown in Table 7.) At this aspect ratio, although the times of charge transfer increases with increasing values of $n$, the peak value of $Q_{S C}$ in each period is far smaller than that of $Q_{\text {tribo }}$. Especially when $n$ is larger than 5, the transfer of charges is unidirectional after the first few cycles, so $Q_{S C \text {-rectified }}$ doesn't have a multiplication effect in these cycles. As a result, $Q_{S C \text {-rectified }}$ starts to drop with an increasing value of $n$. Because of the reverse influence of $n$ in the two different regions, there is an optimum value of $n$ that yields the maximum $Q_{S C \text {-rectified. }}$ As shown in Fig. 4f, for a total geometric aspect ratio, the optimum value of $n$ is about 4, in which the geometric aspect ratio of each unit is 2 . This calculation shows that it is critically important to have this optimum value of $n$ to generate the optimum charge when this kind of TENG is being used to charge a battery or capacitor.

\subsubsection{Grating Structure with Unequal-length Plates}

In the above section, we mainly discussed the design of grating TENGs with two plates of equal length. In such a structure, the top units will gradually slide out from the bottom grating due to lateral displacement. This feature is favourable for a high open-circuit voltage (because of the small side capacitance), but results in non-periodic signals. To avoid this situation, the length of the bottom plate that is stationary during operation can be increased to fully cover the sliding region of the top plate, which forms another 
structure of grating TENGs with plates of unequal length. As a typical example, the length of the bottom part is set to $2 L$, which is twice the length of top part. Thus, the sliding displacement is maintained as $L$. In this structure, because of the charge conservation in contact electrification, the tribo-charge density of Dielectric $1(-2 \sigma)$ is twice as high as that of Dielectric $2(\sigma)$. Because Dielectric 1 is always sliding within the range of Dielectric 2, $Q_{S C}, V_{O C}$ and $C$ will be periodic. In addition, the $Q_{S C}, V_{O C}$ and $C$ curves all have mirror symmetry in each single period, which can be mathematically shown by the following set of equations.

$$
\begin{gathered}
V_{O C}(x+2 k l)=V_{O C}(x) \\
V_{O C}(2 k l-x)=V_{O C}(x) \\
C(x+2 k l)=C(x) \\
C(2 k l-x)=C(x) \\
Q_{S C}(x+2 k l)=Q_{S C}(x) \\
Q_{S C}(2 k l-x)=Q_{S C}(x)
\end{gathered}
$$

Because of the periodicity and mirror symmetry, we only need to simulate the TENG for its first half period. As for the electrode structure, the grating electrode still provides a better performance than the plate electrode in this case, which can be obtained from a similar discussion and derivation as above. Therefore, in the following discussion, we mainly focus on the unequal-length grating TENGs with the grating electrode.

\subsubsection{Influence of dielectric thickness}

Unlike equal-length grating TENGs, the choice of dielectric thickness can significantly influence $Q_{S C}$. Under ideal conditions ( $l / d$ is sufficiently large), $Q_{S C}$ and $\eta_{C T}$ of this grating TENG can be given by: 


$$
\begin{gathered}
Q_{S C}=\left(2-\frac{1}{1+\frac{d_{1} \varepsilon_{r 2}}{d_{2} \varepsilon_{r 1}}}\right) n \sigma w(x-2 k l), \\
2 k l \leq x \leq(2 k+1) l \quad(k \in N) \quad(53 \mathrm{a}) \\
Q_{S C}=\left(2-\frac{1}{1+\frac{d_{1} \varepsilon_{r 2}}{d_{2} \varepsilon_{r 1}}}\right) n \sigma w(2 k l+2 l-x), \\
(2 k+1) l \leq x \leq(2 k+2) l(k \in N) \quad(53 \mathrm{~b}) \\
\eta_{C T}=\left(1-\frac{1}{2} \frac{1}{1+\frac{d_{1} \varepsilon_{r 2}}{d_{2} \varepsilon_{r 1}}}\right) \frac{x-2 k l}{l}, \\
2 k l \leq x \leq(2 k+1) l \quad(k \in N) \quad(54 \mathrm{a}) \\
\eta_{C T}=\left(1-\frac{1}{2} \frac{1}{1+\frac{d_{1} \varepsilon_{r 2}}{d_{2} \varepsilon_{r 1}}}\right) \frac{2 k l+2 l-x}{l}, \\
(2 k+1) l \leq x \leq(2 k+2) l \quad(k \in N) \quad(54 \mathrm{~b})
\end{gathered}
$$

where $n$ is defined as the number of units in top dielectrics. From the above equations, it is observed that $Q_{S C}$ and $\eta_{C T}$ decrease dramatically if the ratio of $d_{2} / d_{1}$ increases, which is because of the non-zero value of $Q_{0}$ from the not totally overlapped surface when $x=0$. To minimize this effect, as shown in Equation 53, the ratio $d_{2} / d_{1}$ needs to be reduced. An effective design is to eliminate the dielectric layer on the bottom plate and use a conductive material as both electrode and tribo-layer, in which $d_{2}$ can be regarded as 0 . In order to validate this, the performance of this conductor-to-dielectric structure $\left(d_{2}=0\right)$ where $n=4$ is compared with the dielectric-to-dielectric TENG structure $\left(d_{2}=d_{1}\right)$ as shown in Fig. 13b-c. The detailed parameters for this calculation are listed in Table 8. 
The peak values of $Q_{S C}, \eta_{C T}$ and $V_{O C}$ from the conductor-to-dielectric TENG are all higher than the dielectric-to-dielectric TENG. Thus, the conductor-to-dielectric design is favourable for efficient charge transfer, from the point of view of device structure. Therefore, we will mainly focus on this design in the following discussion.

\subsubsection{Influence of number of grating units $(n)$}

With the above basic understanding of the output characteristics of the unequal-length grating TENG, we can discuss the influence of the most important design parameter: the number of grating units. The output performance of a TENG with different $n$ is calculated and plotted in Fig. 14. When $n$ increases in the small region, since the aspect ratio of each grating unit $\left(l / d_{1}\right)$ is still large enough, the transferred charge amount in one half-cycle stays almost the same as $Q_{\text {tribo. }}$. Therefore, since the total accumulated charges $\left(Q_{S C \text {,rectified }}(x=L)\right)$ equals to $2 n$ times the amount of transferred charge in one half-cycle, the total accumulated charges and average current magnitude increase quasi-linearly with $n$ in its small value region, as shown in Fig. 14b. However, when $n$ continues to increase, the non-ideal edge effect becomes much more significant due to the decrease in the aspect ratio in each unit, resulting in a decreased number of transferred charges in each half-cycle. Although the number of charge transfer cycles still increases, the elevation slope of the total accumulated charges decreases dramatically. When $n$ is sufficiently large, the slope of the total accumulated charges is close to 0 and the average current no longer increases. Additionally, finer pitches also contribute to a significant increase in the side capacitance between the top and bottom electrodes. Therefore, in Equation 3, $V_{O C}$ will significantly drop with increased $n$, as shown in Fig. 14d. Unlike the case in equallength grating TENG, the $V_{O C}$ and $Q_{S C}$ curves in each half-cycle are always monotonic, 
even when $n$ is sufficiently large. The peak value of the $V_{O C}$ and $Q_{S C}$ curves are always in the middle position of each period. With these characteristics, the unequal-length grating TENGs can have applications as a self-powered sensor for actively measuring the displacement and velocity with high accuracy and resolution.

With the above basic output characteristics, the load performance of these TENGs was numerically calculated, from which the total harvested energy was plotted corresponding to different load resistances, as shown in Fig. 14e and f. Since finer pitches yield a larger inherent TENG capacitance, the optimum resistance shifts significantly to lower values. In addition, unlike the equal-length grating TENGs, an optimum value of $n$ exists (about 108 through interpolation) for the unequal-length grating TENGs, which yields the maximum total harvested energy. With this optimum $n$, the aspect ratio is 3.37 for each grating unit. This optimum $n$ originates from the complicated behavior of $V_{O C}$ and $I_{S C}$. When $n$ first increases, the average value of $I_{S C}$ increases more significantly than $V_{O C}$ decreases, so the harvested energy will increase. However, when $n$ continues increasing, the growth rate of $I_{S C}$ decreases dramatically due to the non-ideal edge effect. Therefore, the total harvested energy starts to decrease when the decreasing $V_{O C}$ becomes the dominant factor. This critical aspect ratio for the individual dielectric unit that yields the largest total energy mainly depends on the motion process and the relative dielectric constant of Dielectric 1. We can take the unequal-length grating TENG at constant velocity as an example. Under this condition, it can be mathematically proved that this critical aspect ratio is only dependent on the relative dielectric constant of Dielectric 1 (independent on $d_{1}, L$, and $v$ ). 


\subsection{Electrostatic shield effect in single-electrode triboelectric nanogenerators}

In previous chapter, we have seen that the electrostatic shield effect is the main reason for the degraded performance of SETENGs.[8] Therefore, effective designs will try to minimize the influence of this electrostatic shield effect. In the following section, we will study the influences of the electrode gap distance and their area size on the output performance and elucidate the optimization strategy for SETENGs. [8]

\subsubsection{Effect of electrode gap distance}

Unlike attached-electrode TENGs, SETENGs have a fixed gap between their two electrodes, which are independent of the motion of Dielectric 1. This leads to an approximately fixed inherent capacitance $C_{0}$, which is critical to the performance of the whole device. To understand the influence of the gap $(g)$, the fundamental properties of the SETENG, i.e. $V_{O C}$ and $Q_{S C}$, are calculated for SETENGs with different gap distances $(\mathrm{g})$, as shown in Figure $\mathbf{3} \mathrm{a}$ and $\mathrm{b}$. In this calculation, all of the other parameters used are the same as shown in Table 1 except the gap distance, which is specified in each figure. At the same moving distance $(x), V_{O C}$ rises up monotonically with the increase of $g$ while $Q_{S C}$ decreases. When $g$ is large enough, the raise speed $V_{O C}$ is quite slow and $V_{O C}$ approaches to its saturation value. Similarly, when $g$ approaches to $0, Q_{S C}$ also approaches to its saturation value.

This influence of $g$ can be interpreted utilizing the equivalent circuit built above. When $g$ increases, $C_{b}$ and $C_{0}$ decreases significantly while $C_{a}$ is barely affected. Therefore, $C_{2}$ and $C_{3}$ decrease significantly while $C_{1}$ maintains almost the same value. In addition, the decrease of $C_{3}$ is in a higher rate than that of $C_{2}$. Therefore, from Equation 18-20, $V_{O C}$ monotonically increases with the increase of $g$ while $Q_{S C}$ monotonically decreases to 0 . 
Besides the basic trend of $V_{O C}$ and $Q_{S C}$, their saturation behavior can be theoretically understood as well. First, the saturation behavior of $V_{O C}$ when $g$ is sufficiently large is discussed. When $g$ is sufficiently large, $C_{b}$ and $C_{0}$ approach to 0 at the same rate while $C_{a}$ is almost unaffected. Therefore, $C_{2}$ and $C_{3}$ approach to 0 with its ratio approaching to 1 while $C_{l}$ approaches to $C_{a}$. Thus, $V_{O C}$ saturates to the value of $\sigma w l /\left(2 C_{\mathrm{a}}\right)$ when $g$ is large enough.

The saturation behavior of $Q_{S C}$ can also be interpreted. When $g$ decreases to $0, C_{o}$ is getting close to infinity and $C_{a}$ equals to $C_{b}$. Thus, both $C_{1}$ and $C_{2}$ are approaching to $C_{a} / 2$. As a result, $Q_{S C}$ approaches to its saturation value $\sigma w l / 2$.

Besides the fundamental property, the influence of the gap on the load characteristics is investigated as well. The output current, voltage, and transit power peak were calculated under different load resistances for SETENGs with different $g$, as shown in Figure 15c-e. A smaller gap provides a larger inherent capacitance, leading to the decrease of the optimum resistance. The relationship between the maximum transit power and the gap distance is plotted in Figure 15f. An optimum gap about $1.54 \mathrm{~cm}$ is observed to generate the maximized transit power.

\subsubsection{Effect of area size (length)}

Besides the gap between the electrodes, another important design parameter is the area size (represented by the length $l$ in this $2 \mathrm{D}$ model) of the SETENG. To systematically study its influence, $V_{O C}, Q_{S C}$, and $\eta_{C T}$ of the SETENG with different length $l$ is calculated through the COMSOL software, as shown in Figure 16a-c. All of the parameters utilized in this calculation are the same as listed in Table 1, except that the value of $x_{\max }$ utilized 
in the calculation is $2 \mathrm{~cm}$. From the calculation results, for any given $x$, there exists a specific $l$ to generate the largest $V_{O C}$. In addition, when $x$ increases, this $l$ for the largest $V_{O C}$ also increases. $Q_{S C}$ should have been proportional to the length $l$, but this is true only when $l$ is sufficiently small. When $l$ is large enough, the $Q_{S C} l$ curve shifts downwards dramatically, which shows that $\eta_{C T}$ decreases significantly when $l$ is large.

The complicate behavior of the influence of $l$ to outputs of SETENGs can be understood from the equivalent circuit built above. The capacitances $C_{a}, C_{b}$, and $C_{0}$ are first calculated, which are all 2D parallel electrode capacitances. For an non-ideal 2D parallel electrode capacitance considering the edge effect, its capacitance value can be shown as the following equation,[26]

$C=\frac{\varepsilon_{0} w l}{d}\left\{1+\frac{d}{\pi l}\left\{1+\ln \left[1+\frac{2 \pi l}{d}+\ln \left(1+\frac{2 \pi l}{d}\right)\right]\right\}\right\}=\frac{\varepsilon_{0} w l}{d}\left[1+\alpha\left(\frac{d}{l}\right)\right]$

where $l$ is its length, $w$ is its width and $d$ is the separation distance between the two parallel electrodes. Inside Equation 25, the edge effect is reflected by the function $\alpha(y)$ :

$\alpha(y)=\frac{y}{\pi}\left\{1+\ln \left[1+\frac{2 \pi}{y}+\ln \left(1+\frac{2 \pi}{y}\right)\right]\right\}$

With $C_{a}, C_{b}$, and $C_{0}$ calculated through the above equation, $C_{1}, C_{2}$, and $C_{3}$ can be analytically calculated utilizing Equation 15-17. Thus, from the calculated $C_{1}, C_{2}$, and $C_{3}$, $V_{O C}, Q_{S C}$, and $\eta_{C T}$ can be analytically calculated through Equation 18-20. Their behaviors at the two $l$ limits are shown below.

When $l$ approaches to $0(x / l$ and $g / l$ is very large):

$V_{O C}=\frac{\sigma}{2 \varepsilon_{0}} \pi l(57 \mathrm{a}) \quad Q_{S C}=\frac{\sigma w l}{2} \quad(57 \mathrm{~b}) \quad \eta_{C T}=\frac{1}{2}$

When $l$ approaches to infinity $(x / l$ and $g / l$ is close to 0$)$ : 
$V_{O C}=\frac{\sigma g x \ln (l)}{\pi \varepsilon_{0} l}$

$\eta_{C T}=\frac{x}{\pi l} \ln (l)$

From Equation 57a and 58a, the behavior of $V_{O C}$ can be quite easily understood. When $l$ is small, $V_{O C}$ is proportional to $l$ and increases with the increase of $l$. But when $l$ is large enough, $V_{O C}$ satisfies Equation 58a and starts to drop. Therefore, there is an optimum $l$ at which $V_{O C}$ reaches its maximum. When $x$ or $g$ increases, a larger range of $l$ can satisfy the condition for Equation 57, so that the $l$ providing the maximum $V_{O C}$ rises. For $Q_{S C}$, when $l$ is small (condition of Equation 57), $Q_{S C}$ is proportional to $l$ and increases dramatically with the increase of $l$. When $l$ is sufficiently large (condition of Equation 58), $Q_{S C}$ is proportional to $\ln (l)$, resulting in a much lower slope of $Q_{S C^{-}} l$ curve. From the $Q_{S C}$ behavior, $\eta_{C T}$ is close to $50 \%$ when $l$ is small and decays to 0 when $l$ is sufficiently large. This analysis clearly explains the FEM calculation results shown in Figure 16.

Besides these fundamental characteristics, the resitive load characteristics of the contactmode SETENG are studied as well. Because $V_{O C}$ and $\eta_{C T}$ of SETENGs with infinite $l$ are 0 , an optimum $l$ of $4.7 \mathrm{~cm}$ is observed to generate the maximum total transit power, as shown in Figure 16d and 16e. In addition, the optimum resistance increases first then starts to decrease when $l$ increases. This is because when $l$ first increases, $V_{O C}$ increases at a faster speed than $I_{S C}$, Region II of the single electrode shifts to a higher resistance range and the optimum resistance increases. ${ }^{[12]}$ When $l$ further increases, $V_{O C}$ starts to decrease and $I_{S C}$ continues increasing. Thus, the optimum resistance starts to decrease. ${ }^{[12]}$ Moreover, the peak power density is monotonically lowered when $l$ increases, which is because larger area $l$ lowers $\eta_{C T}$. Thus, unlike the attached-electrode TENGs, SETENGs are not optimized for direct scaled-up processing. 


\subsubsection{Effect of unit spacing for scale up}

Finally, the effect of connecting several SETENGs in parallel is discussed, which is another way to scale-up the output from SETENGs besides increasing the area size. For a three-SETENG system, a simulation was performed at OC condition through FEM calculation, in order to study the influence of the spacing between each SETENG. The total $Q_{S C}$ generated by the three SETENGs with different spacing is shown in Figure $16 \mathrm{f}$. When the unit spacing is small, electric field from each SETENG will interfere, resulting in a decrease of $\eta_{C T}$ for each SETENG, which is similar to the effect of increasing $l$ on one SETENG. Only when their spacing is large enough, the mutual influence will be minimized, so that there will be little decrease on $\eta_{C T}$. From Figure 16f, to reach the total charge transfer amount more than $270 \%$ of that for each SETENG, a minimum unit spacing of $1.24 \mathrm{~cm}$ is observed, about 2.5 times the length of an individual SETENG. Therefore, a large enough spacing is critical for the scale-up of the output through parallel connection.

\subsection{Superior linear characteristics of contact-mode freestanding TENGs and their application in harvesting vibration energy}

As discussed above, the most advantage of this CFTENG structure is its superior linearity. [15] The linear dependence of both $V_{\mathrm{OC}}$ and $Q_{\mathrm{SC}}$ on $x$ makes it an ideal medium to transform mechanical vibration signal to electrical signal. Besides, the constant inherent capacitance makes it a linear and time-invariant device. Thus, the electrical signal output of this device is compatible with all the currently available signal processing techniques, 
making it easy to accomplish post-processing of the obtained electrical signal. Therefore, this structure could have wide applications in self-powered vibration detection.

Besides the self-powered sensor application, this superior linearity is also beneficial in vibration energy harvesting applications as well. To fully uncover its advantages, we design a device structure that can harvest vibration energy under both freestanding-mode and traditional attached-electrode mode, as shown in Fig. 17a. When metal 1 and metal 2 are directly utilized as two electrodes and metal 3 and 4 are suspended with no output terminal, this device is working in the freestanding-mode (shown in the right solid line). If metal 1 and metal 4 are connected to form Node A, metal 2 and metal 3 are connected to form Node B and then Node A and Node B are utilized as the output terminals to connect with external load circuits, the device is working in traditional attached-electrode mode, which is equivalent to two parallel-connected attached-electrode TENGs. The reason to connect metal 1 and 4 rather than metal 1 and 2 to form the output node is to ensure the polarity of these two TENGs is consistent.

As a comparison, $Q_{\mathrm{SC}}$ under both two operation-modes are numerically calculated utilizing the same parameters and plotted in Fig. 17c. Although for both of these twomodes, their $Q_{\text {sc-final }}$ (short-circuit transferred charge amount when a full separation is reached) can reach $2 \sigma S$, the shape of $Q_{\mathrm{sc}}$ is completely different. For the freestanding mode, $Q_{\mathrm{sc}}$ changes linearly with $x$, ensuring a relative high slope in the whole $x$ range. However, for the traditional attached-electrode mode, $Q_{\text {sc }}$ only has a huge slope at the two ends. When $x$ is between $0.05 g$ to $0.95 g, Q_{\mathrm{sc}}$ is nearly a constant. Therefore, when the traditional attached-electrode mode is utilized to harvest vibration energy with vibration amplitude which is not large enough to ensure the freestanding layer to fully contact with 
the two electrodes (which is always the case in practical application), the total shortcircuit transferred charges will be strongly limited. As an example, this device is utilized to harvest a harmonic vibration whose vibration amplitude is $g / 4$ and center position is $x$ $=g / 2$. The steady state electrical outputs under these two modes are numerically calculated through the TENG simulator utilizing the periodic boundary condition. As shown in Fig. 17c, the amount of transferred-charges of the freestanding mode is more than 70 times higher than the attached-electrode mode in all the resistance ranges. In addition, the optimum energy harvested from the freestanding-mode is several orders larger than the attached-electrode mode. From the above comparison, we can conclude that the linearity of the freestanding mode is highly beneficial for harvesting the mechanical energy from the vibration that cannot ensure good contact of the moving object and the two electrodes, which will also save energy loss from the inelastic collision and increase the energy conversion efficiency.

\subsection{Optimization for sliding-mode freestanding TENGs}

\subsubsection{Tolerance to the freestanding height}

The most significant advantage of the SFTENG structure is its excellent tolerance to the freestanding height $h$.[15] This loosens the requirement of the sliding object and reduces the energy loss due to the friction. The influence of the freestanding height on the output performance of dielectric SFTENGs is first investigated. Besides the parameter specified in the figure, the value of all the other parameters are the same as listed in Table 2. From Fig. 18a, $Q_{\mathrm{SC} \text {,final }}$ decreases when $h$ increases, which is mainly because of the reduce of the difference between $\left(C_{2}(k) / C_{1}(k)\right)_{x=0}$ and $\left(C_{2}(k) / C_{1}(k)\right)_{x=g+l}$. Specifically, when $h=0$, 
$C_{2}(k) / C_{1}(k)$ equals to 0 at the position of $x=0$ and equals to infinity at the position of $x=$ $g+l$, so $Q_{\mathrm{SC}, \text { final }}$ can reach $\sigma w l$. When $h$ is large enough, the average distance between the tribo-charge surface and two electrodes will be approximately the same. Thus both $\left(C_{2}(k) / C_{1}(k)\right)_{x=0}$ and $\left(C_{2}(k) / C_{1}(k)\right)_{x=g+l}$ are approximately 1 , resulting in a zero $Q_{\mathrm{sc}, \text { final. }}$. But the decreasing slope with height is much flatter than the attached-electrode STENG case. From the numerical calculation results, the half-life height that $Q_{\mathrm{SC}, \text { final }}$ reduces to its halfvalue for attached-electrode sliding-mode TENGs is $250 \mu \mathrm{m}$, while that for SFTENG structure is about $2 \mathrm{~cm}$. As for the capacitance profile shown in Fig. 18b, the increase of $h$ only slightly decreases the total capacitance since the capacitance in the dielectric SFTENGs is mainly from the direct capacitance between metal 1 and metal 2. For the $V_{\mathrm{OC}}$ profile of the dielectric SFTENG shown in Fig. 18c, it almost has the same trend with $Q_{\mathrm{SC}}$, for the capacitance almost has no dependence with $h$. As for the total energy- $R$ curve, this degradation of both $Q_{\mathrm{SC}}$ and $V_{\mathrm{OC}}$ will lead to the decrease of the maximum harvested energy when $h$ increases. However, the optimum resistance stays almost the same, which is because of the nearly same inherent capacitance for different $h$.

For metal SFTENGs, their height characteristics have some similarity with dielectric SFTENGs, but it will still have several differences. Since the basic electrostatic induction mechanism in the metal SFTENG system is very similar to that of dielectric SFTENG system, the change of $Q_{\mathrm{SC}}$ profiles with height is similar to that of dielectric SFTENG system. However, there are still many differences. When $h$ increases, the influence of the metal layer on the electrostatic system reduces and both $C_{\mathrm{f} 1}$ and $C_{\mathrm{f} 2}$ significantly decrease. Thus, as shown in Fig. 19b, the total capacitance at the middle position is largely affected when $h$ first increases from 0 . When $h$ is large enough, the total capacitance starts to be 
dominated by the direct parasitic capacitance between the two electrodes and is nearly independent of $x$. At this time, the metal SFTENGs are indistinguishable with the dielectric SFTENGs. As for the open-circuit voltage shown in Fig. 19c, the metal SFTENGs also show a very complicated profile, which is because both $Q_{\mathrm{SC}}$ and $C$ have strong dependence on the freestanding height. $V_{\mathrm{OC}-\text { final }}$ will decrease because of a reduced $Q_{\text {SC-final }}$ and an almost non-changed $C$ in that region. However, at the middle region, $V_{\mathrm{OC}}$ will increase first when $h$ increases then decreases when $h$ is already large enough. This is because the decreasing speed of the serial connection of $C_{\mathrm{f} 1}$ and $C_{\mathrm{f} 2}$ is faster than the decreasing speed of $Q_{\mathrm{SC}}$ in this region. When $h$ is small and $x$ is in the middle range, this serial connection dominates the total capacitance and $V_{\mathrm{OC}}$ has this unusual increase. As for the total harvested energy, it also has the similar decay trend as the dielectric SFTENGs. However, since the average total capacitance drops with the increase of $h$, the optimum resistance shifts to the right when $h$ first increases and finally stays on the value which is almost the same as the dielectric SFTENGs.

\subsubsection{Influence of the gap between electrodes}

Besides the freestanding height $h$, another important design parameter is the gap distance between the electrodes $(g)$. The capacitance between the electrodes is strongly dependent on the gap distance. Therefore, the gap distance will have strong effect on the total output characteristics.

The effect of $g$ on the total output performance for dielectric SFTENGs under $h=0$ conditions is studied first and plotted in Fig. 20. Besides the parameters specified in the figure, all the other parameters is the same as listed in Table 2. Since $h$ equals to 0 , the 
ratio $C_{2}(k) / C_{1}(k)$ is still 0 when $x=0$ and infinity when $x=g+l$. Therefore, the charge transfer efficiency $\eta_{\mathrm{CT}}$ can still reach $100 \%$ for all the $g$ values and $Q_{\text {SC-final }}$ will not change with $g$. However, the capacitance between the two electrodes decreases when $g$ increases, as shown in Fig. 20b. Thus, through equation 3, $V_{\mathrm{OC}-\text { final }}$ when a full separation is reached will increase with $g$. Therefore, considering the total energy harvested in one cycle, the dielectric SFTENG with a higher $g$ will generate a larger total energy with a higher optimum resistance. However, for dielectric SFTENGs with a higher $g$, their cycle $(T)$ is also longer when the average velocity stays the same. Therefore, the average power (defined as equation 59a) will have a different trend with the total harvested energy, as shown in Fig. 20d.

$$
P_{a v g}=\frac{R \int_{0}^{T} I^{2} d t}{T}(59 a) \quad T=\frac{2(l+g)}{v}
$$

When $g$ first increases from 0 , the cycle length increases only a little because $l$ still dominates the total cycle length. Therefore, the increase of $V_{\text {OC-final }}$ dominates the total average power and average power increases with $g$. However, when $g$ is already very large, the decreasing slope of the total capacitance is flattened. Therefore, the increase of $V_{\mathrm{OC}-\text { final }}$ cannot catch up with the increasing speed of $T$ and the average power begins to drop. Thus, an optimum gap is observed to generate the largest average power.

For metal SFTENGs under $h=0$ conditions, similar results have also been observed. The charge transfer efficiency $\eta_{\mathrm{CT}}$ can still reach $100 \%$ for all the $g$ values and $Q_{\text {SC-final }}$ will not change with $g$. The total capacitance of metal SFTENGs also decrease with the increase of $g$, but this decrease contributes from two reasons. The first reason is the same 
as dielectric SFTENGs, which will lead to the decrease of the direct parasitic capacitance between these two electrodes. Besides, when $g$ increases, the region when the freestanding layer are overlapped with both of the two electrodes decreases, leading a decrease of the serial connection of $C_{\mathrm{f} 1}$ and $C_{\mathrm{f} 2}$. When $g$ is larger than $l$, the region that the freestanding layer is overlapped with both electrodes no longer exists and the serial connection of $C_{\mathrm{f} 1}$ and $C_{\mathrm{f} 2}$ can be neglected. The influence of $g$ on $V_{\mathrm{OC}}$ of metal SFTENGs is very similar to the case in dielectric SFTENGs. $V_{\mathrm{OC}-\text { final }}$ will increase with the increase of $g$. Also, similar to the dielectric SFTENGs, the optimum resistance still increases when $g$ increases and there is an optimum gap to reach the highest average power.

Besides the $h=0$ case we discussed above, the influence of the gap distance will be much more complicated if there is a non-zero freestanding height. When $h$ is non-zero, the capacitance ratio $C_{2}(k) / C_{1}(k)$ is no longer 0 when $x=0$ and no longer infinity when $x=$ $g+l$. And if the gap decreases, the difference of the capacitance ratio $C_{2}(k) / C_{1}(k)$ between $x=0$ position and $x=g+l$ position will reduce as a response, resulting in a decrease of $Q_{\text {SC-final. }}$ The $3 \mathrm{~d}$ plot of $Q_{\text {SC-final }}$ under different $h$ and $g$ for dielectric SFTENG is shown in Fig. 21a. Consistent with our theoretical analysis, when $g$ increases, the slope of $Q_{\text {SC- }}$ final $h$ curve decreases and the dielectric SFTENG has more tolerance to the height $h$. In addition, $Q_{\text {sc-final }}$ will increase with the increase of $g$ when $h$ is a non-zero constant and the slope of $Q_{\mathrm{SC}-\text { final- }} g$ curves increase when $h$ goes up. As for the average power shown in Fig. 21 b, there is still an optimum gap value when $h$ increases. This optimum gap at which the highest average power is reached increases with $h$, as a result of better tolerance of height for larger gap devices. For metal SFTENGs, similar results have also been observed. 


\section{Material Selection and its relationship with structural optimization}

Besides the structural optimization strategy we proposed above, another factor in improving TENG performance is to optimize the tribo-pair materials.[25] The major effect of changing tribo-pair materials on the output performance is the change in tribocharge density $(\sigma)$. Additionally, different materials will lead to different relative dielectric constants as well, but this will have a negligible effect on the output performance.

First, different tribo-pair materials will result in different levels of tribo-charge density, which will strongly influence the output performance. Due to the superposition principle of electric potential, $V_{O C}$ is proportional to $\sigma$ while the capacitance term $(C)$ is independent on $\sigma$. Thus, the output parameters (including $Q, V$, and $I$ ) are all directly proportional to $\sigma$. Therefore, $\sigma$ will only affect the magnitude of the output parameters, but not their shape with respect to both time or load resistance. Therefore, all the structural parameters (including optimum resistance and optimum aspect ratio) have no dependence on $\sigma$. Thus, the structural design is completely decoupled from $\sigma$, which is the main parameter to target while selecting tribo-pair materials.

Besides, as shown in the above discussion of TENGs, material selection will have an impact on the structural parameters by changing the effective dielectric thickness (defined as $d_{i} / \varepsilon_{r i}$ ). However, this effect is always trivial in tribo-pair material selection because of the following two reasons. First, the relative dielectric constant of the most commonly utilized tribo-pair materials does not vary too much (always in the range of 24), so compared to the influence of $\sigma$, changing these dielectric constants does not 
significantly affect the output performance. Second, as the effective dielectric thickness directly affects the structural parameters, the impact of changing the relative dielectric constants can always be compensated by changing the dielectric thickness on the same scale. Thus, in practical TENG design, optimizing $\sigma$ is always the only target of material selection, which is a fully decoupled process from structural optimization.

To choose materials that can provide a higher $\sigma$, we need to select one material which is tendency to lose electrons and the other one which is tendency to gain electrons. However, because the detailed mechanism of contact electrification is still under investigation, we can only rely on some empirical results, tabulated in the triboelectric series.[27] Tribo-pair materials are always selected from the two ends of the triboelectric series. Additionally, nanostructures on the surface of the tribo-pair materials and ion injection have been experimentally proven to be an effective way to improve $\sigma .[28,29]$

\section{Summary and perspective}

In summary, this paper presents the fundamental theory of triboelectric nanogenerators. Triboelectric nanogenerators have inherent capacitive behavior and their governing equation is their $V-Q-x$ relationship. There are two capacitance formed between the tribocharged dielectric surface and the two metal electrodes, respectively. The ratio of these two capacitances changes with the position of this dielectric surface, inducing electrons to transfer between the metal electrodes under SC condition. This is the core working mechanism of triboelectric generators. When triboelectric generators are connected with resistive loads, a "three-working-region" behavior is shown because of the impedance match between the generator and the load. Besides, when triboelectric generators are 
utilized to charge a capacitor with a bridge rectifier in multiple motion cycles, it is equivalent to utilizing a DC voltage source with an internal resistance to charge the capacitor. Four fundamental modes of triboelectric generators are thoroughly analyzed to show their different output characteristics. The attached-electrode contact-mode and sliding-mode triboelectric nanogenerators need to maintain the minimum gap size to be much smaller than the effective dielectric thickness. The electrostatic shield effect of the primary electrode is the main design consideration of single-electrode triboelectric generators. Contact-mode freestanding triboelectric generators have superior linear characteristics and sliding-mode freestanding triboelectric generators have excellent height tolerance. For the most-complicated grating structure, increasing the number of grating units to get a finer pitch will generally improve the output performance. However, when the pitch is very fine, the edge effect begins to dominate, resulting in degradation of performance when the number of units continues to increase. Thus, there exists an optimum number of grating units, and an optimum unit aspect ratio.

The proposed triboelectric nanogenerator theory can be utilized as an important guidance in their rational design to reach an optimum performance for practical applications. The current area power density of TENGs has reached $313 \mathrm{~W} / \mathrm{m}^{2}$ and their volume energy density has reached $490 \mathrm{~kW} / \mathrm{m}^{3}$. Utilize the above TENG theory and optimization technique, we suggest that this already very high energy density can still be increased by 10 times through rational structural design. Through material optimization (to improve the tribo-charge density to the air-breakdown limit), the energy density can be even further improved. Besides, similar to the development of CMOS integrated circuits and systems, the fully-integrated energy harvesting systems that contain triboelectric 
generators, power management circuits, signal processing circuits, energy storage elements, and/or load circuits are essential for the practical applications of the TENGs. The system-level design techniques and the post-processing circuit topologies still require theoretical research. Finally, since hybridizing triboelectric generators and batteries to form a self-charged power pack has already shown potential applications in self-power energy systems, the optimization techniques of such self-powered system is still required.

Acknowledgement:

Research was supported by BES DOE, NSF, Airforce, Samsung, SKKU (Korea), MANA NIMS (Japan), and the Knowledge Innovation Program of the Chinese Academy of Sciences (KJCX2-YW-M13). The authors also thank Dr. Ying Liu and Dr. Sihong Wang for their kind discussion on the manuscript. All of the materials presented have been published and proper references have been cited, and some published figures and possibly text have been used for this review article.

Reference:

[1] F.R. Fan, Z.Q. Tian, Z.L. Wang, Nano Energy, 1 (2012) 328-334.

[2] Z.L. Wang, Acs Nano, 7 (2013) 9533-9557.

[3] Y.N. Xie, S.H. Wang, S.M. Niu, L. Lin, Q.S. Jing, J. Yang, Z.Y. Wu, Z.L. Wang, Adv. Mater., 26 (2014) 6599-6607.

[4] G. Zhu, J. Chen, T.J. Zhang, Q.S. Jing, Z.L. Wang, Nat Commun, 5 (2014) 3426.

[5] Y.S. Zhou, G. Zhu, S.M. Niu, Y. Liu, P. Bai, Q.S. Jing, Z.L. Wang, Adv. Mater., 26 (2014) 1719-1724.

[6] S.M. Niu, Y. Liu, S.H. Wang, L. Lin, Y.S. Zhou, Y.F. Hu, Z.L. Wang, Adv. Mater., 25 (2013) 6184-6193.

[7] S.M. Niu, S.H. Wang, L. Lin, Y. Liu, Y.S. Zhou, Y.F. Hu, Z.L. Wang, Energy Environ. Sci., 6 (2013) 3576-3583.

[8] S.M. Niu, Y. Liu, S.H. Wang, L. Lin, Y.S. Zhou, Y.F. Hu, Z.L. Wang, Adv. Funct. Mater., 24 (2014) 3332-3340. 
[9] S.M. Niu, Y.S. Zhou, S.H. Wang, Y. Liu, L. Lin, Y. Bando, Z.L. Wang, Nano Energy, 8 (2014) 150-156.

[10] J. Stoer, R. Bulirsch, Introduction to numerical analysis, 3rd ed., Springer, New York, 2002.

[11] S.H. Wang, L. Lin, Z.L. Wang, Nano Lett., 12 (2012) 6339-6346.

[12] G. Zhu, C.F. Pan, W.X. Guo, C.Y. Chen, Y.S. Zhou, R.M. Yu, Z.L. Wang, Nano Lett., 12 (2012) 4960-4965.

[13] F. Saurenbach, D. Wollmann, B.D. Terris, A.F. Diaz, Langmuir, 8 (1992) 1199-1203. [14] L.H. Lee, J. Electrostat., 32 (1994) 1-29.

[15] S.M. Niu, Y. Liu, X.Y. Chen, S.H. Wang, Y.S. Zhou, L. Lin, Y.N. Xie, Z.L. Wang, Adv. Mater., (2015). submitted

[16] S.H. Wang, L. Lin, Y.N. Xie, Q.S. Jing, S.M. Niu, Z.L. Wang, Nano Lett., 13 (2013) 2226-2233.

[17] G. Zhu, J. Chen, Y. Liu, P. Bai, Y.S. Zhou, Q.S. Jing, C.F. Pan, Z.L. Wang, Nano Lett., 13 (2013) 2282-2289.

[18] L. Lin, S.H. Wang, Y.N. Xie, Q.S. Jing, S.M. Niu, Y.F. Hu, Z.L. Wang, Nano Lett., 13 (2013) 2916-2923.

[19] G. Zhu, Y.S. Zhou, P. Bai, X.S. Meng, Q.S. Jing, J. Chen, Z.L. Wang, Adv. Mater., 26 (2014) 3788-3796.

[20] G. Zhu, W.Q. Yang, T.J. Zhang, Q.S. Jing, J. Chen, Y.S. Zhou, P. Bai, Z.L. Wang, Nano Lett., 14 (2014) 3208-3213.

[21] Y. Yang, Y.S. Zhou, H.L. Zhang, Y. Liu, S.M. Lee, Z.L. Wang, Adv. Mater., 25 (2013) 6594-6601.

[22] S.H. Wang, Y.N. Xie, S.M. Niu, L. Lin, Z.L. Wang, Adv. Mater., 26 (2014) 28182824.

[23] L. Lin, S.H. Wang, S.M. Niu, C. Liu, Y.N. Xie, Z.L. Wang, Acs Appl. Mater. Inter., 6 (2014) 3038-3045.

[24] S.M. Niu, Y. Liu, Y.S. Zhou, S.H. Wang, L. Lin, Z.L. Wang, IEEE Trans. Electron Devices, (2015). accepted.

[25] S.M. Niu, S.H. Wang, Y. Liu, Y.S. Zhou, L. Lin, Y.F. Hu, K.C. Pradel, Z.L. Wang, Energy Environ. Sci., 7 (2014) 2339-2349.

[26] Y. Li, Y.H. Li, Q.X. Li, Y.Y. Zi, J. Tsinghua Univ. (Sci. \& Tech.), 43 (2003) 10241026.

[27] A.F. Diaz, R.M. Felix-Navarro, J. Electrostat., 62 (2004) 277-290.

[28] F.R. Fan, L. Lin, G. Zhu, W.Z. Wu, R. Zhang, Z.L. Wang, Nano Lett., 12 (2012) 3109-3114.

[29] S.H. Wang, Y.N. Xie, S.M. Niu, L. Lin, C. Liu, Y.S. Zhou, Z.L. Wang, Adv. Mater., 26 (2014) 6720-6728.

\section{Figures:}




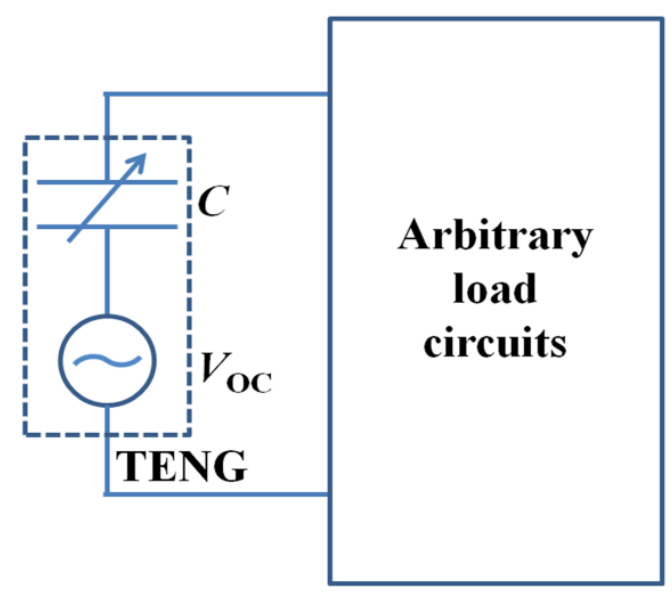

Figure 1. First-order lumped-parameter equivalent circuit model of any triboelectric nanogenerators. Reproduced from reference [9].

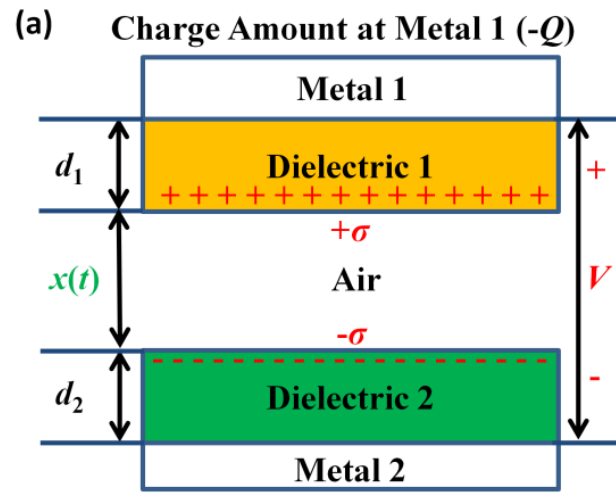

Charge Amount at Metal $2(Q)$ (b)

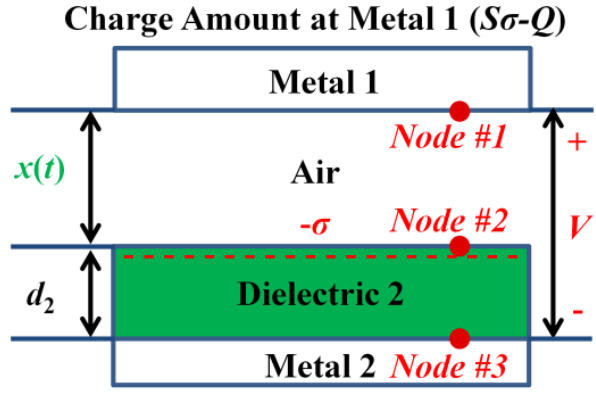

Charge Amount at Metal $2(Q)$ (c)

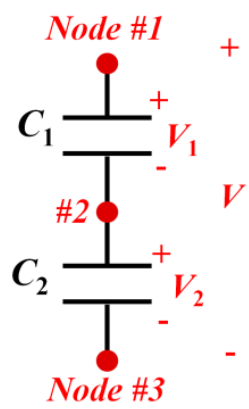

Figure 2. Theoretical models for (a) Dielectric-to-dielectric attached-electrode parallelplate contact-mode TENG and (b) Conductor-to-dielectric attached-electrode parallelplate contact-mode TENG. (c) Equivalent circuit diagram for Conductor-to-dielectric attached-electrode parallel-plate contact-mode TENG. Reproduced from reference [7]. Copyright 2013 Royal Society of Chemistry. 


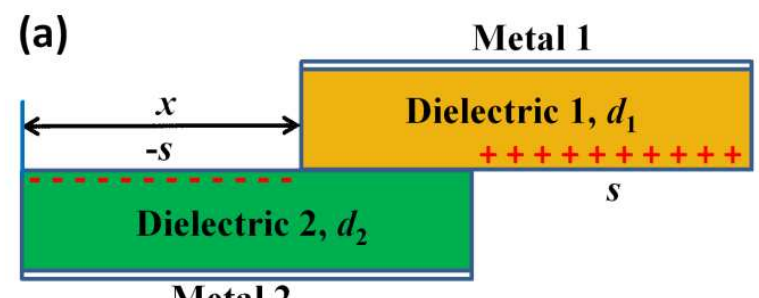

Metal 2

(c)

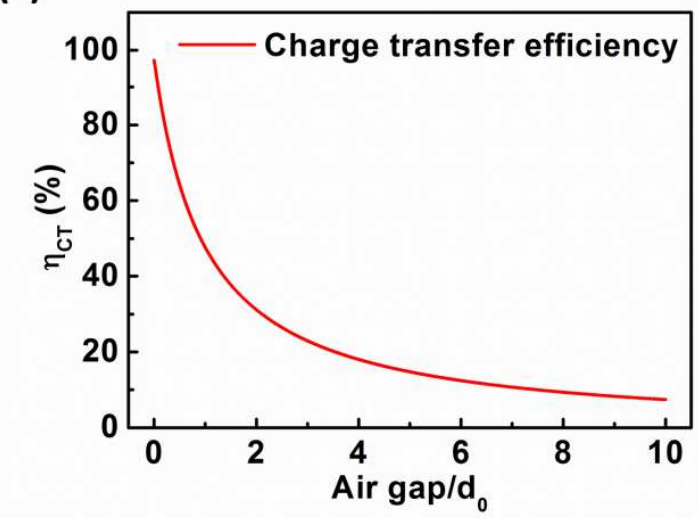

(b)

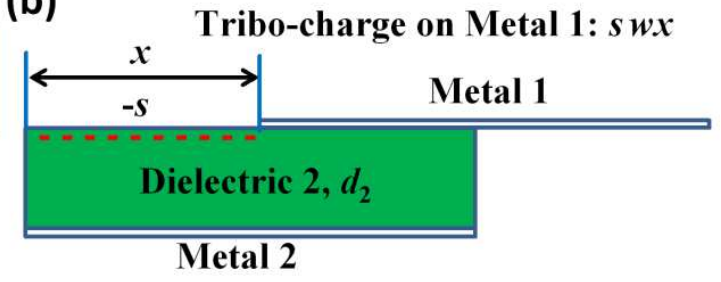

(d)

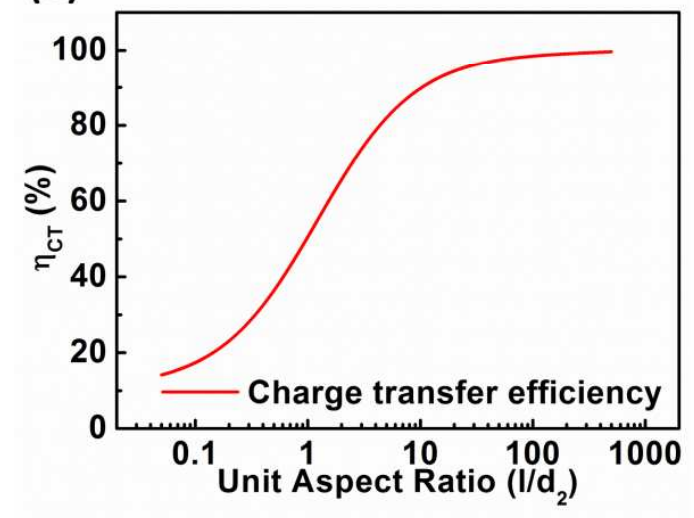

Figure 3. Theoretical models for (a) Dielectric-to-dielectric attached-electrode parallelplate sliding-mode TENG and (b) Conductor-to-dielectric attached-electrode parallelplate sliding-mode TENG. (c-d) Influence of (c) air gap size and (d) dielectric aspect ratio on the charge transfer efficiency of conductor-to-dielectric sliding-mode TENG when a full separation has been reached. The dielectric constant is 2 in this calculation. Reproduced from reference [6]. Copyright from 2013 Wiley. 


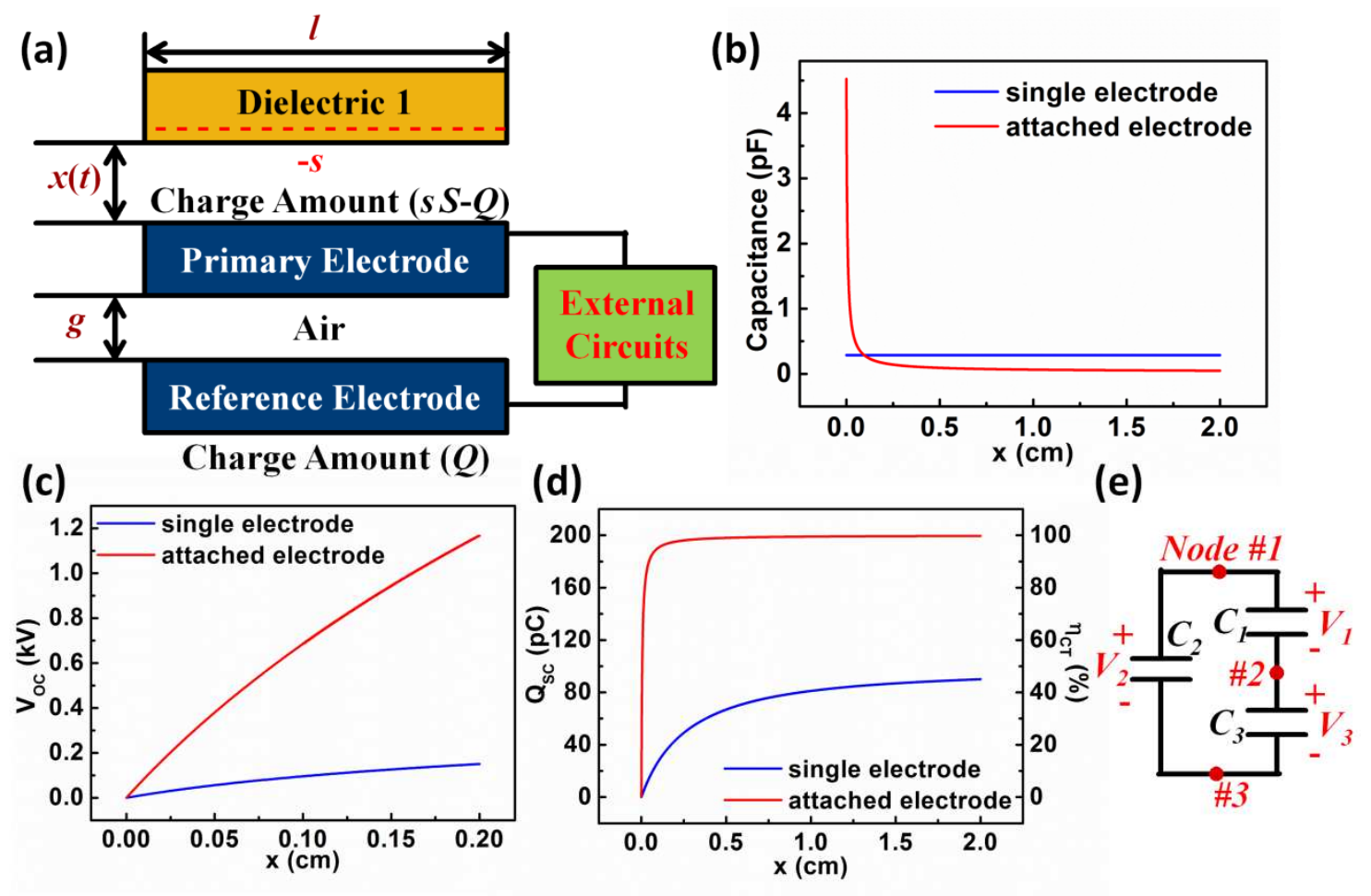

Figure 4. Basic output characteristics of conductor-to-dielectric contact-mode SETENGs.

(a) Structure of the FEM model. (b-d) Calculated (b) capacitance between the two electrodes, (c) open circuit voltage, and (d) transferred charges at short circuit condition of SETENGs at different $x$, together with comparison with results of attached-electrode TENGs. (e) Equivalent circuit model containing three capacitances for the SETENGs under open-circuit (OC) condition. Reproduced from reference [8]. Copyright from 2014 Wiley. 


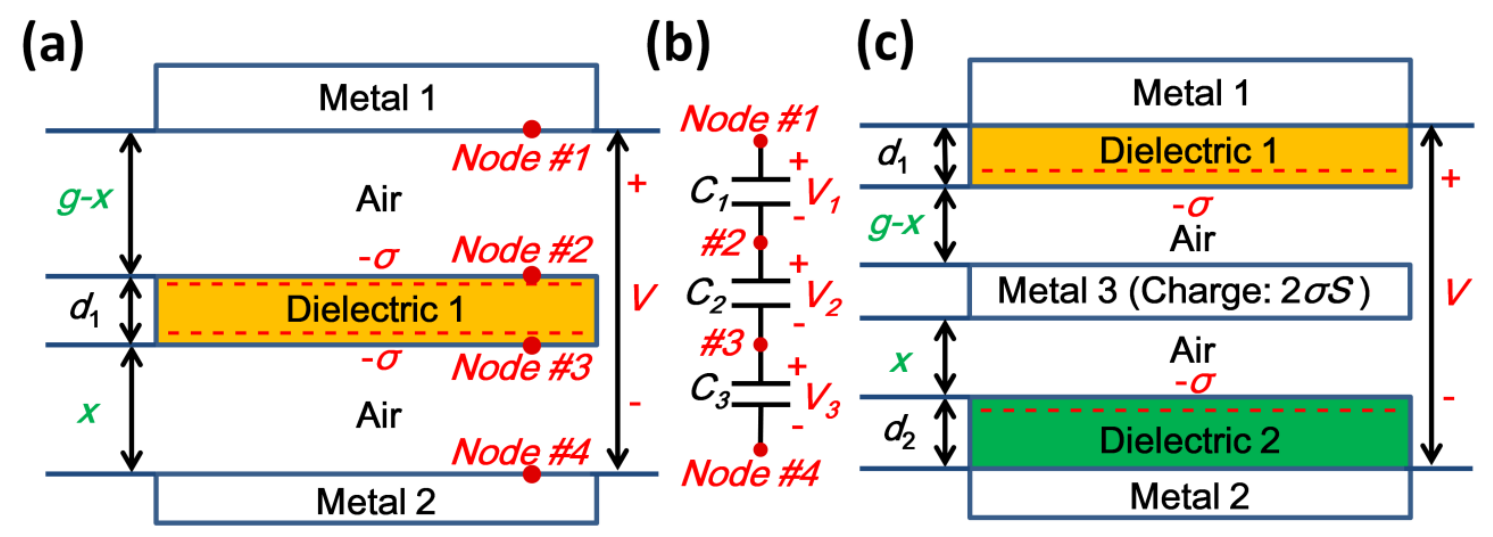

Figure 5. Theoretical models of contact-mode freestanding TENGs. (a) Model of a typical dielectric freestanding layer CFTENG. (b) Equivalent circuit model of the dielectric CFTENG electrostatic system. (c) Model of a typical metal freestanding layer CFTENG. Reproduced from reference [15]. 

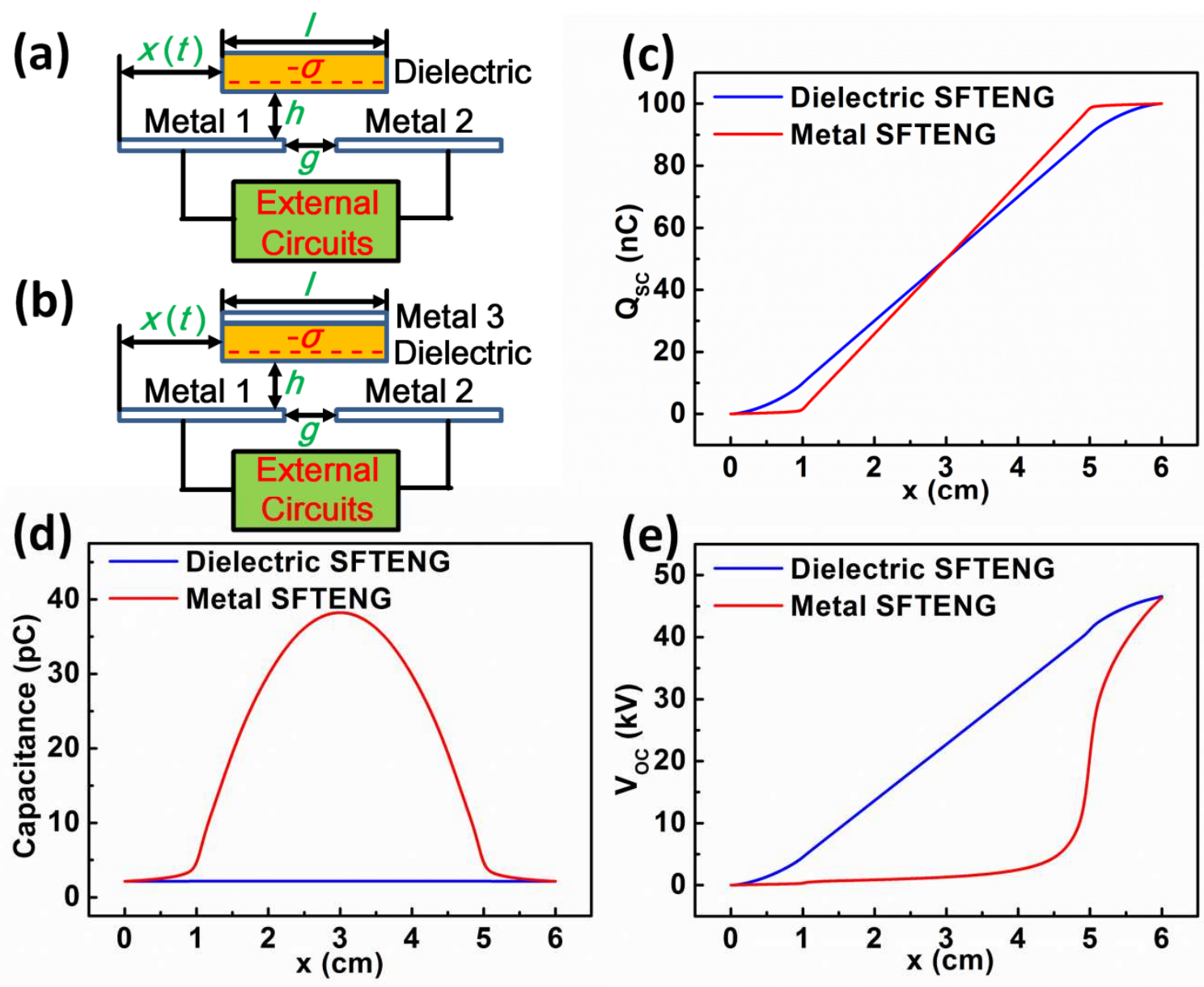

Figure 6. Models and basic output characteristics of sliding-mode freestanding TENGs.

(a) FEM model of a typical dielectric SFTENG. (b) FEM model of a typical metal SFTENG. (c-e) Influence of freestanding layer material on the basic output characteristics: (c) short-circuit transferred charges under MACRS, (d) total capacitance between the two electrodes, and (e) open-circuit voltage under MACRS. Reproduced from reference [15]. 
(a)
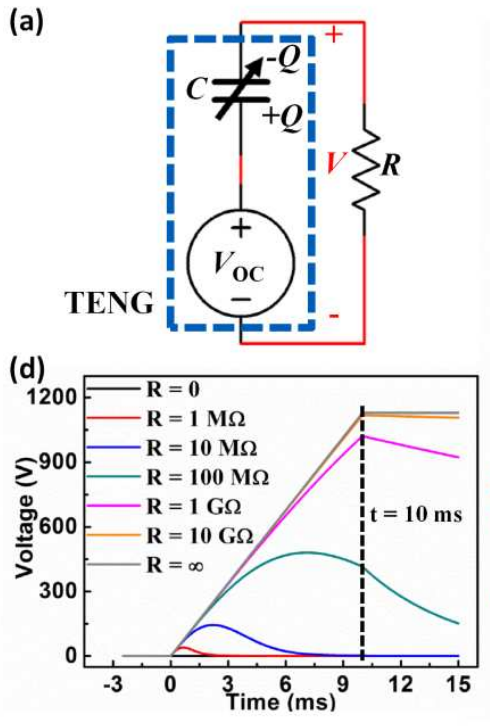

(b)

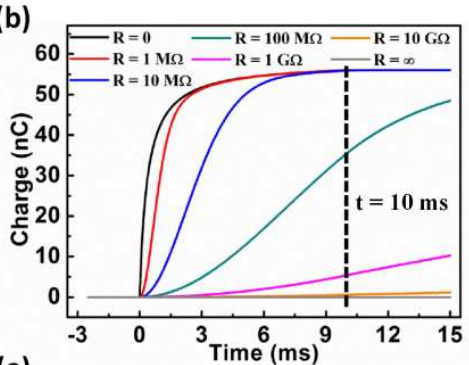

(e)

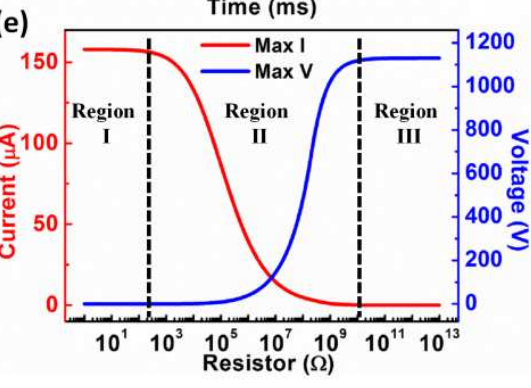

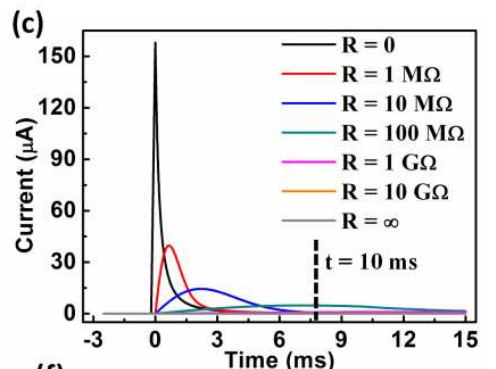

(c)

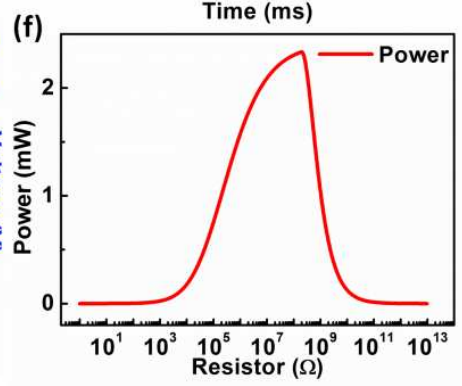

Figure 7. Theoretical calculated resistive output characteristics of attached-electrode contact-mode TENGs under uniform velocity separation. The top plate reaches the maximum separation distance and stops moving at $t=10 \mathrm{~ms}$. (a) Equivalent circuit model of the whole TENG system. (b) Real-time transferred charge-time relationship at different load resistances. (c) Real-time current-time relationship at different load resistances. (d) Real-time voltage-time relationship at different load resistances. (e) The influence of the load resistance on the magnitude of the output current and voltage. Three working regions are marked. (f) The influence of the load resistance on the instantaneous power output. Reproduced from reference [7]. Copyright 2013 Royal Society of Chemistry. 

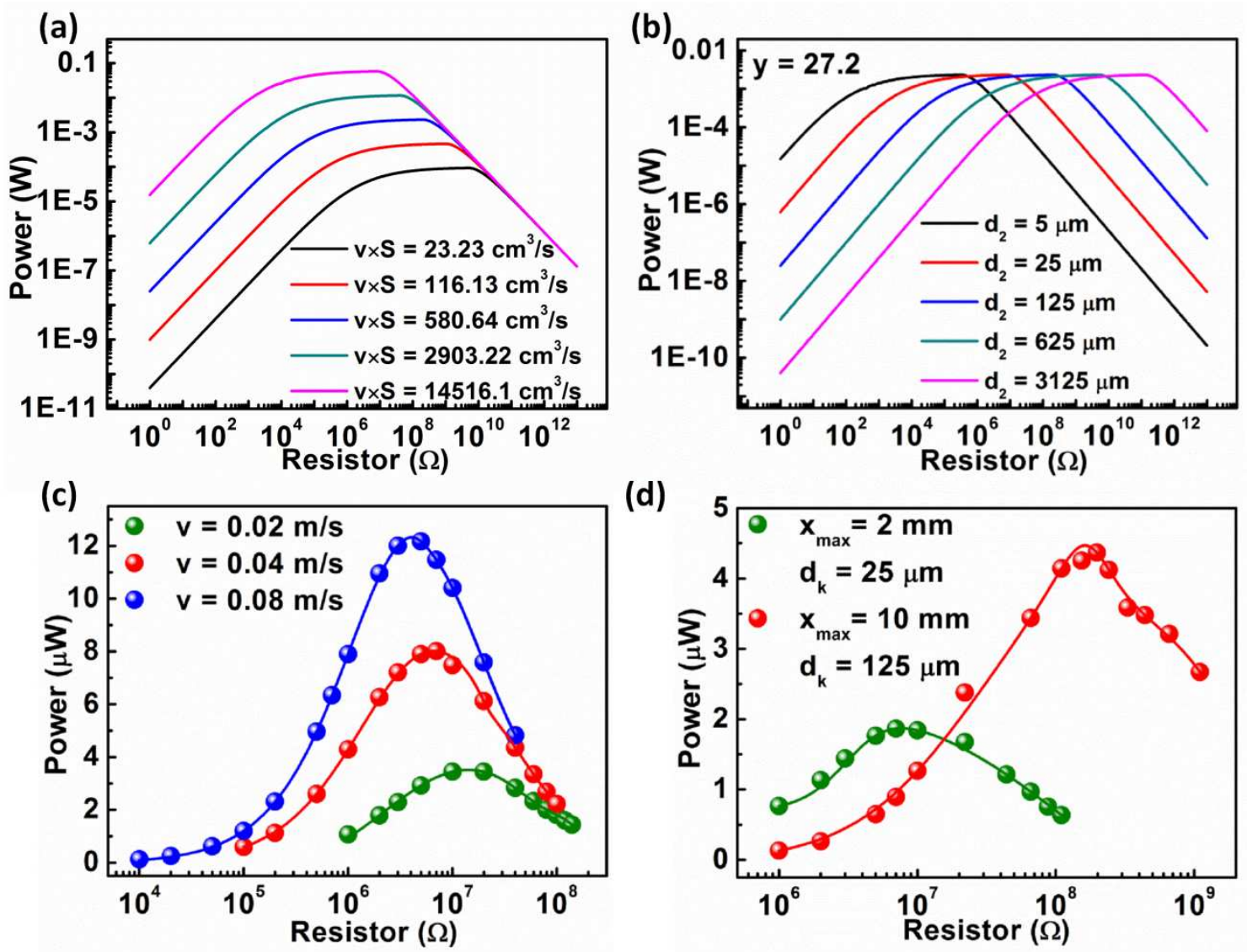

(d)

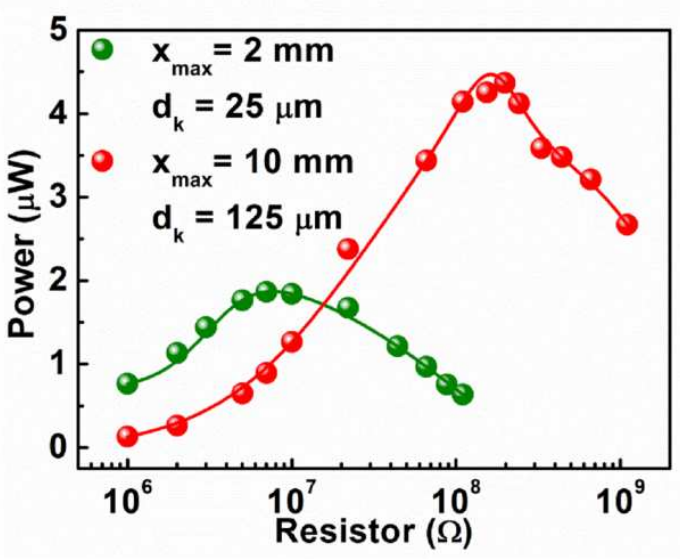

Figure 8. Optimum resistance characteristics of attached-electrode contact-mode TENGs.

(a-b) The relationship of optimum resistance with different TENG parameters under uniform velocity motion. (a) Maximum output power profile with load resistance and at different velocities or area sizes. (b) Maximum output power profile with load resistance under different effective dielectric thickness and gap distances with maintaining their ratio as a constant of 27.2. (c-d) Experimental comparison of the optimum resistances from TENG devices with different parameters. The dots are measured values and the lines are obtained through interpolation of the experimental data. (c) Maximum output power profile with load resistance at different average velocities. (d) Maximum output power profile with load resistance at different Kapton film thickness and gap distances 
with their ratio remaining as constant. Reproduced from reference [7]. Copyright 2013 Royal Society of Chemistry.

(a)

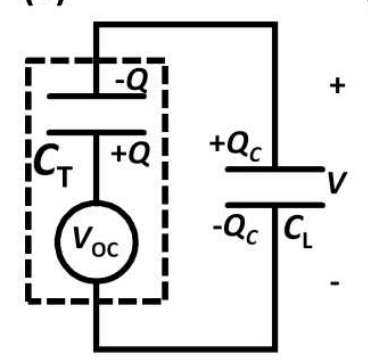

(b)

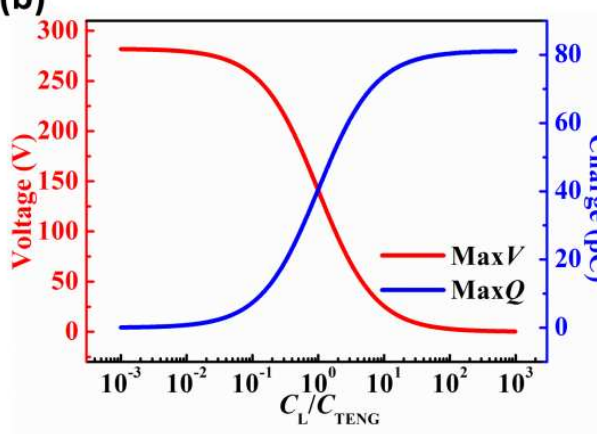

(c)

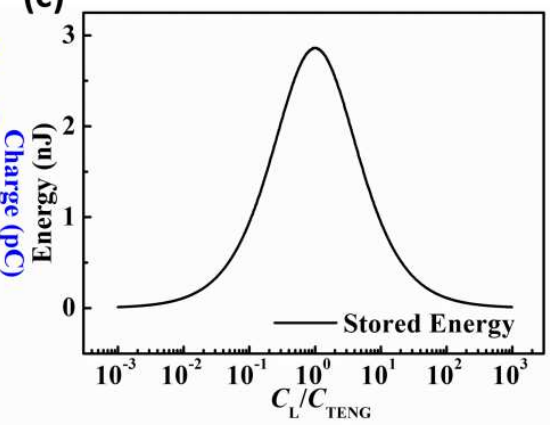

Figure 9. TENG charging characteristics under unidirectional mechanical motion. (a) Equivalent circuit model of TENG-capacitor system of the unidirectional charging calculation. (b) The influence of the load capacitance on the final voltage and charge stored in the load capacitor. (c) Final stored energy profile with load capacitance. Reproduced from reference [24]. Copyright 2015 IEEE. 

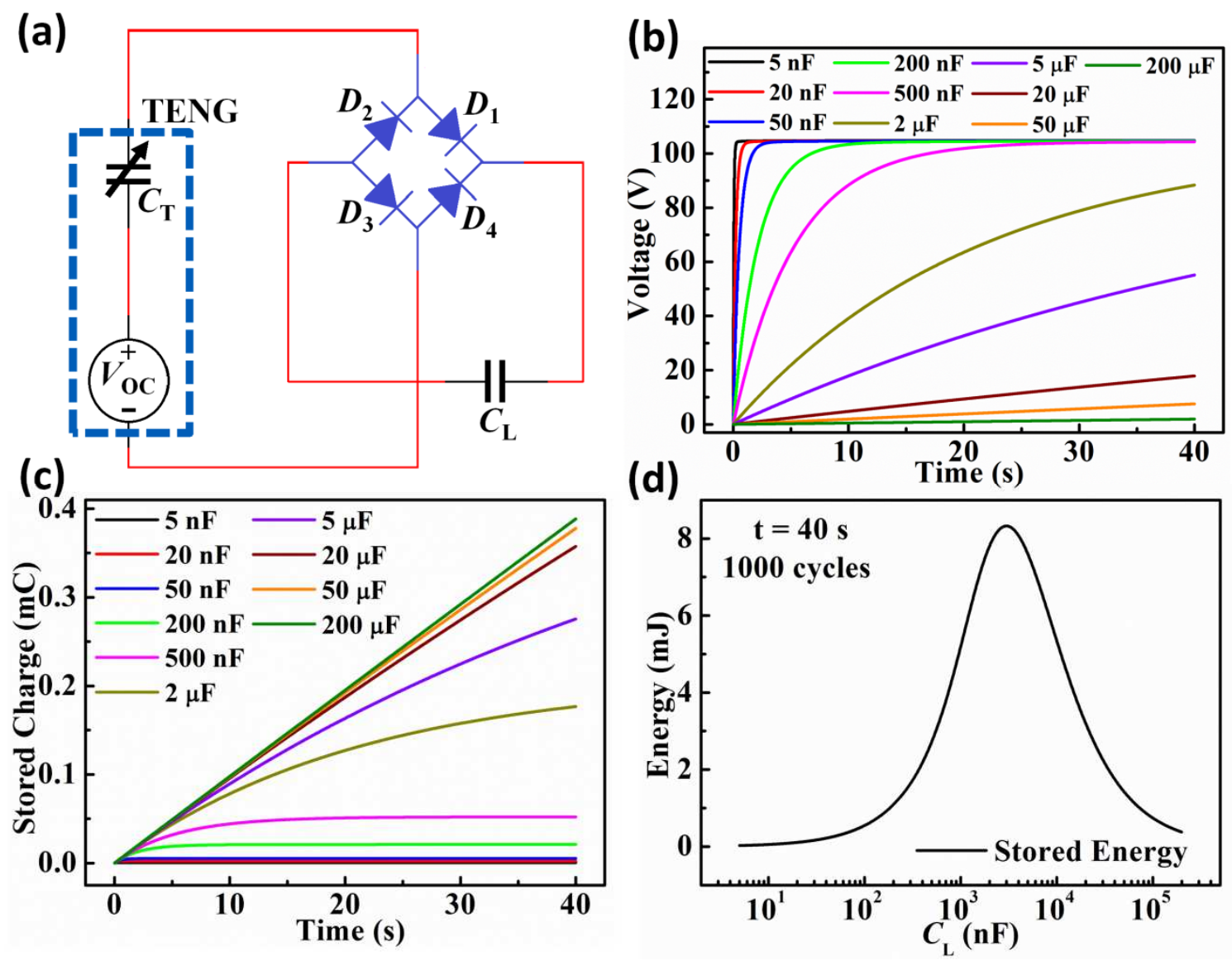

Figure 10. TENG charging characteristics under periodic mechanical motion. (a) Circuit diagram for periodic charging calculation. (b) Voltage-time relationship at different load capacitances. (c) Stored charge-time relationship at different load capacitances. (d) Final stored energy profile with load capacitance. Reproduced from reference [24]. Copyright 2015 IEEE. 
(a) Grating Electrodes:

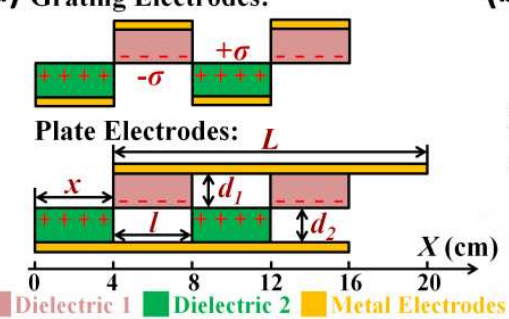

(d)

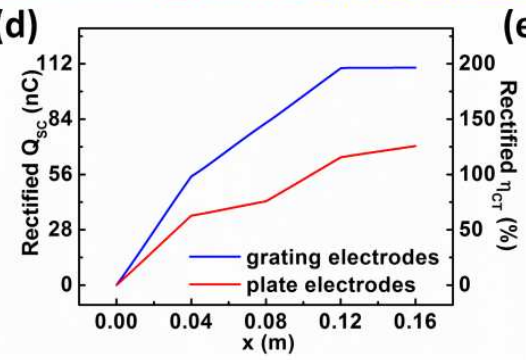

(b)

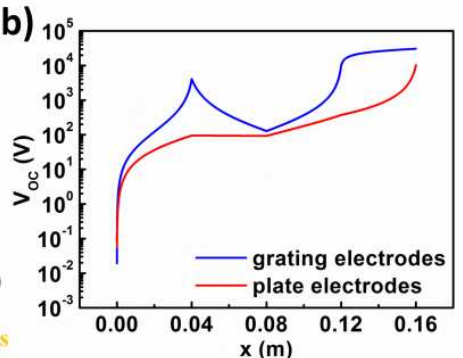

(e)

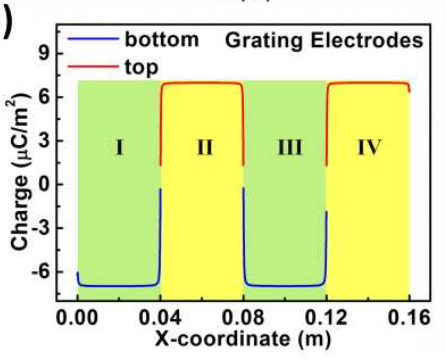

(c)
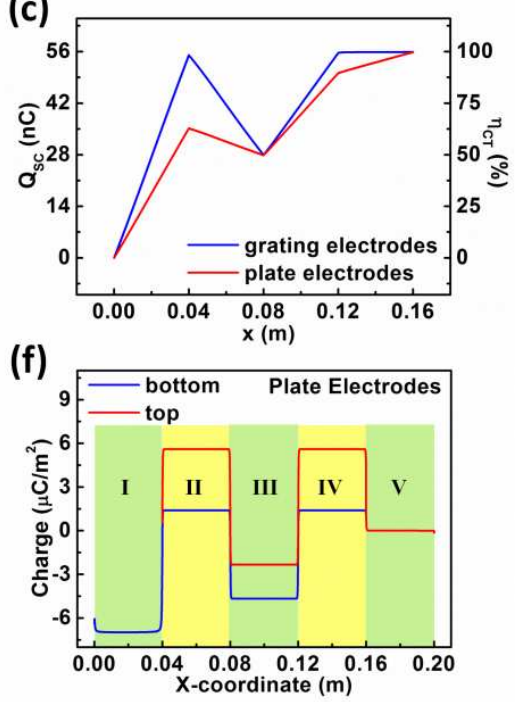

Figure 11. Electrode structure optimization of equal-length grating TENGs. (a) Structure of the FEM model for grating TENGs with grating electrodes and plate electrodes. (b-d) Calculated (b) open circuit voltage, (c) transferred charges at short circuit condition, and (d) rectified transferred charges at short circuit condition for grating TENGs with both grating electrodes and plate electrodes. (e-f) charge distribution at the metal electrodes for (e) grating electrodes and (f) plate electrodes at the case $x=l$. Reproduced from reference [25]. Copyright 2014 Royal Society of Chemistry. 
(a)
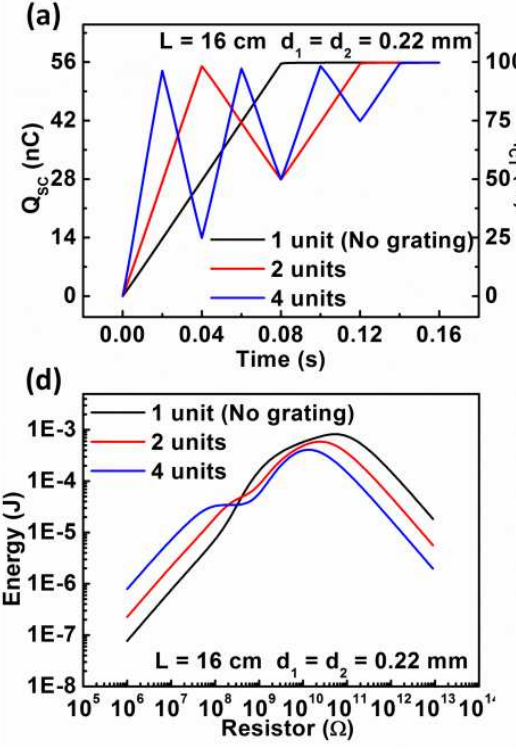

(b)

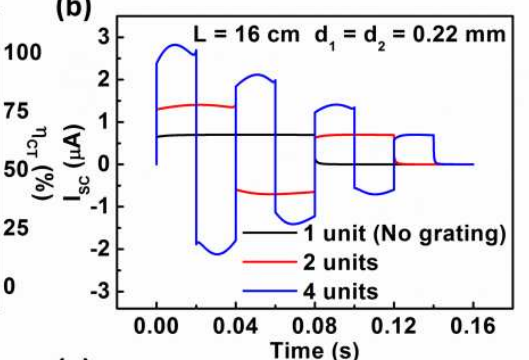

(e)

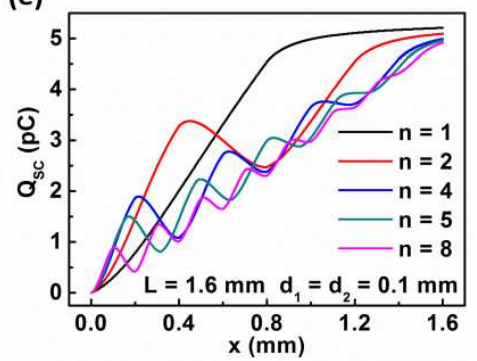

(c)

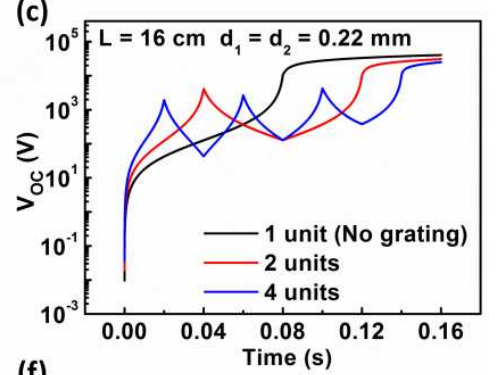

(f)

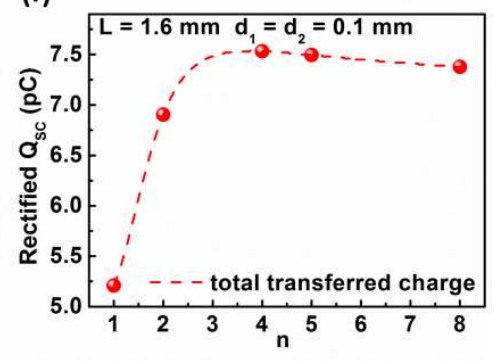

Figure 12. Influence of number of grating on the output performance of equal-length grating TENGs. (a-d) Influence of $n$ in the ideal condition. (a) The relationship between transferred charges at short circuit and time at different $n$. (b) The relationship between current at short circuit and time at different $n$. (c) The relationship between open circuit voltage and time at different $n$. (d) The relationship between generated energy and load resistance at different $n$. (e-f) Influence of $n$ in the non-ideal condition. (e) transferred charges at short circuit profile with moving distance at different $n$. (f) Total transferred charges relationship with $n$. Reproduced from reference [25]. Copyright 2014 Royal Society of Chemistry. 
(a)
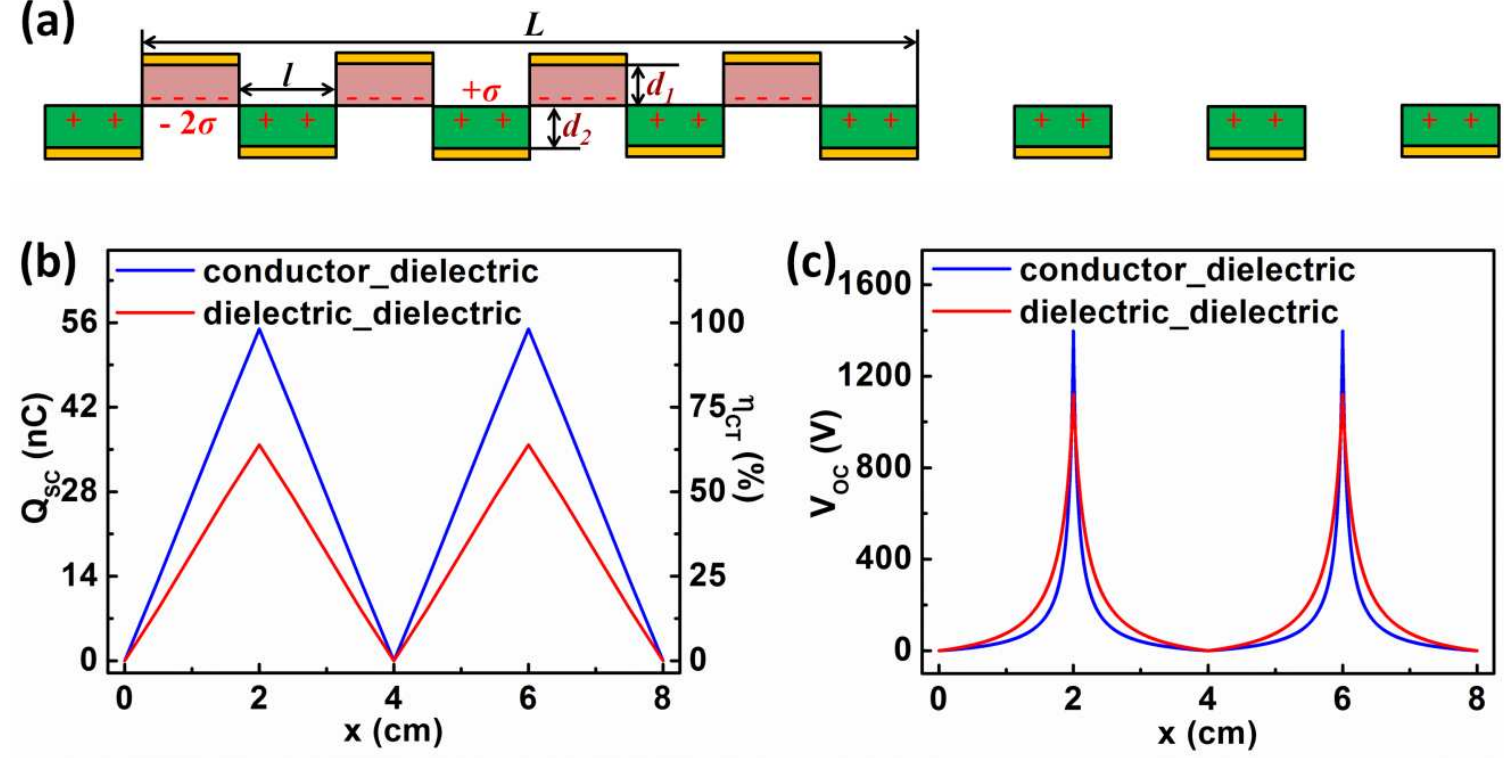

Figure 13. Influence of dielectric thickness on the performance of the unequal-length grating TENGs. (a) FEM model for the unequal-length grating TENGs. (b-c) Comparison of conductor-to-dielectric and dielectric-to-dielectric unequal-length grating TENGs on (b) short circuit transferred charges and (c) open circuit voltage. Reproduced from reference [25]. Copyright 2014 Royal Society of Chemistry. 


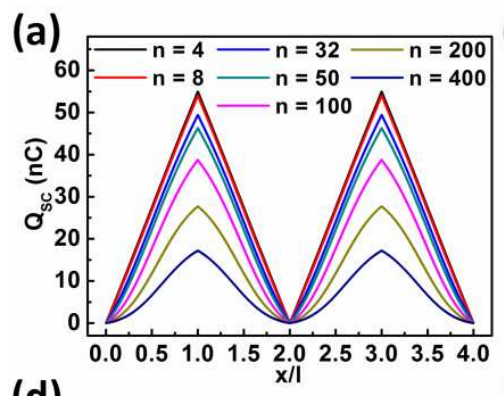

(d)

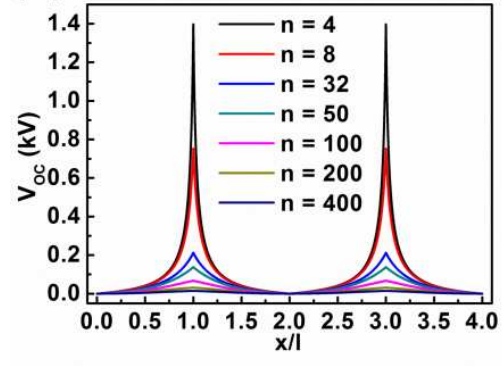

(b)

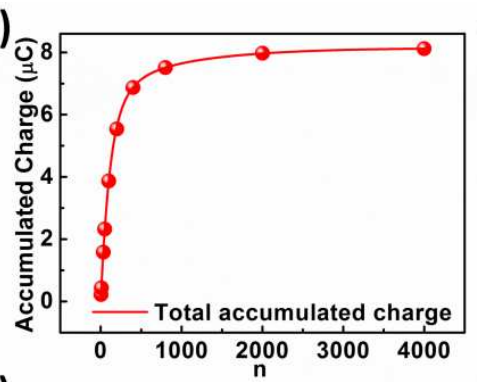

(e)

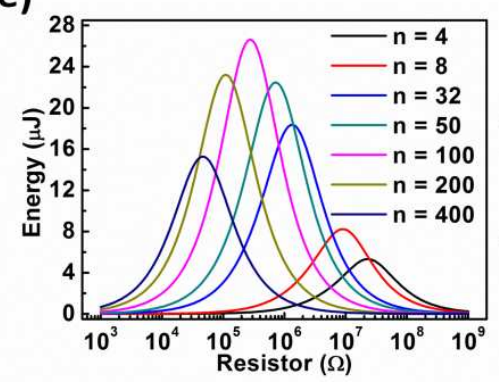

(c)

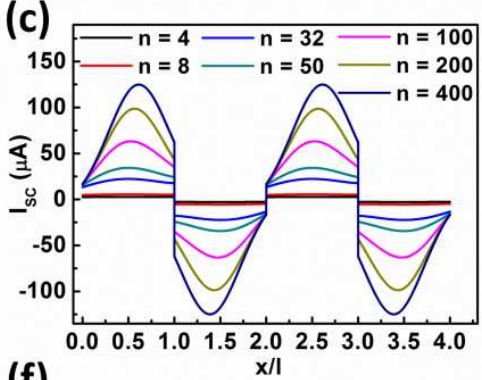

(f)

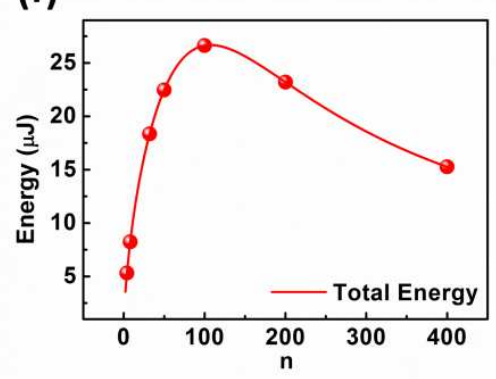

Figure 14. Influence of number of grating for unequal-length grating TENGs. (a) The relationship between transferred charges at short circuit in one period at different $n$. (b) Extracted total transferred charges when $x=L$. (c) The current profile at short circuit in one period at different $n$. (d) The voltage profile at open circuit in one period at different $n$. (e) The relationship between total generated energy and load resistance at different $n$.

(f) Influence of $n$ on the total generated energy. Reproduced from reference [25]. Copyright 2014 Royal Society of Chemistry. 
(a)

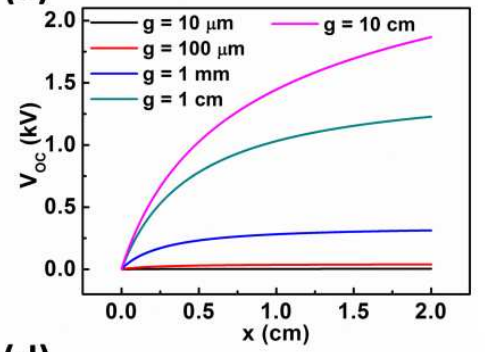

(d)

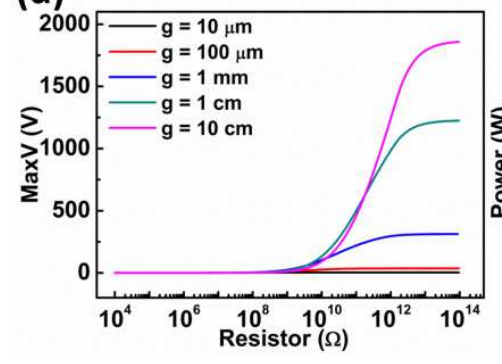

(b)

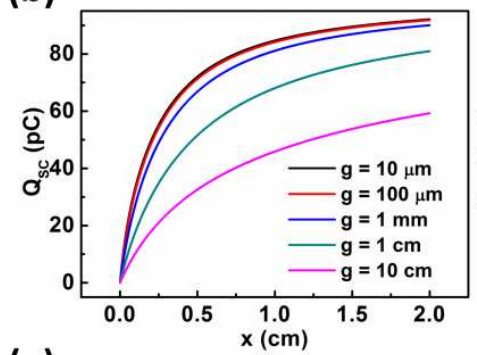

(e)

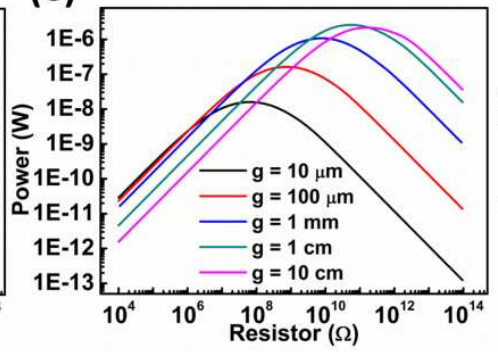

(c)

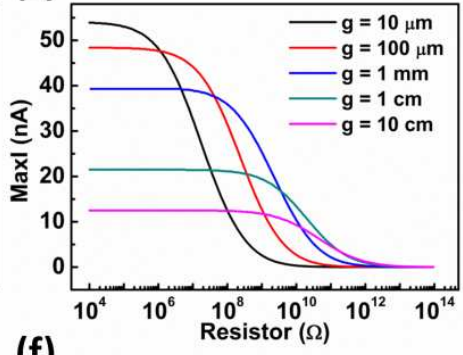

(f)

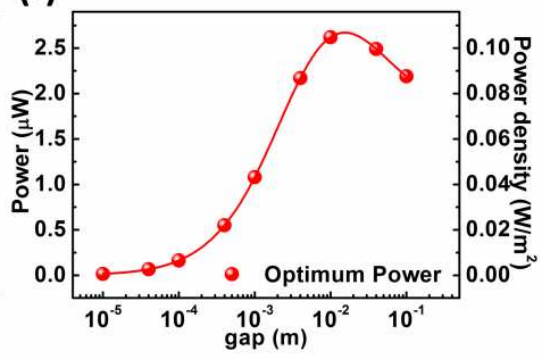

Figure 15. Influence of gap distance $(g)$ on the output performance of the single-electrode TENG. (a) The relationship between open circuit voltage and separation distance at different gap distances. (b) The relationship between transferred charges at short circuit and separation distance at different gap distance. (c-e) Maximum output (c) current profile, (d) voltage profile, and (e) power profile with load resistance at different gap distances. (f) Extracted optimum power profile with gap distance. Reproduced from reference [8]. Copyright from 2014 Wiley. 
(a)

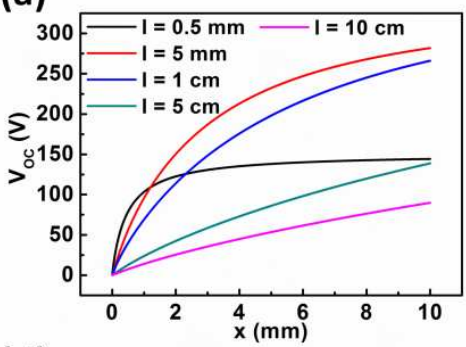

(d)

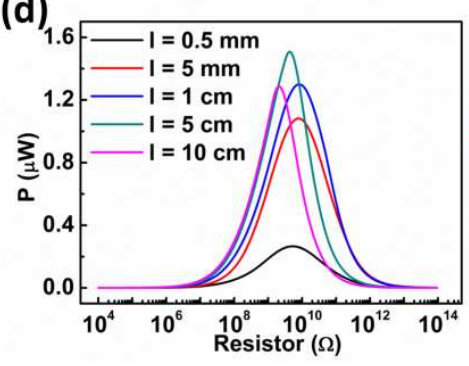

(b)

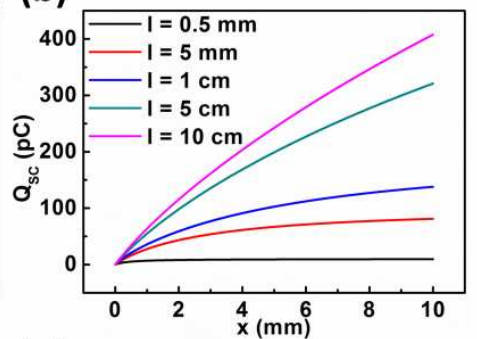

(e)

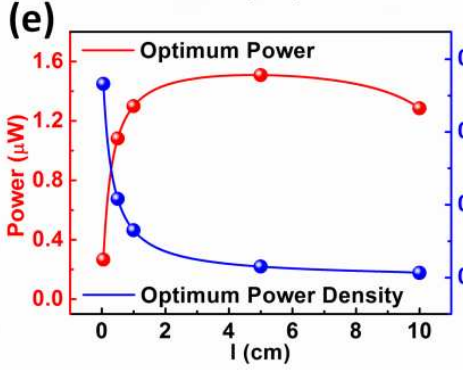

(c)

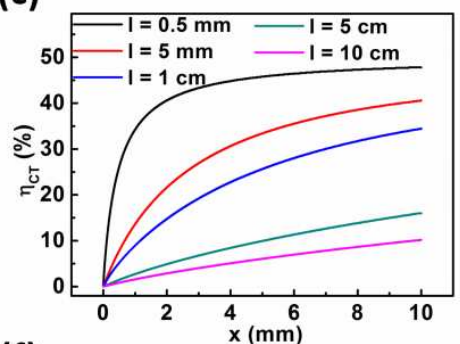

(f)

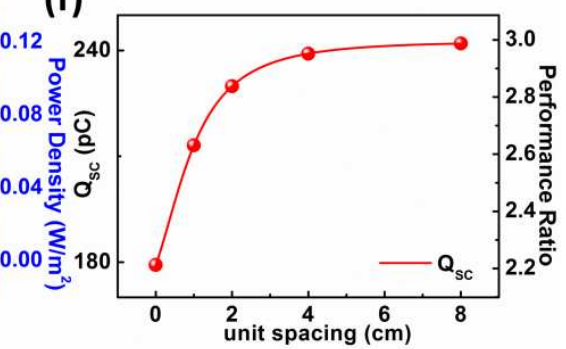

Figure 16. The influence of area size/length on the output characteristics of the singleelectrode TENG. (a) open circuit voltage profiles with separation distances for SETENGs with different lengths. (b) short circuit transferred charges profile with separation distances for SETENGs with different lengths. (c) short circuit charge transfer efficiency profile with separation distances for SETENGs with different lengths. (d) Maximum output power profile with load resistance for SETENGs with different lengths. (e) Extracted optimum power and power density profile with length of SETENG. (f) The influence of unit spacing on the short circuit transferred charges in a three-SETENG system. Reproduced from reference [8]. Copyright from 2014 Wiley. 

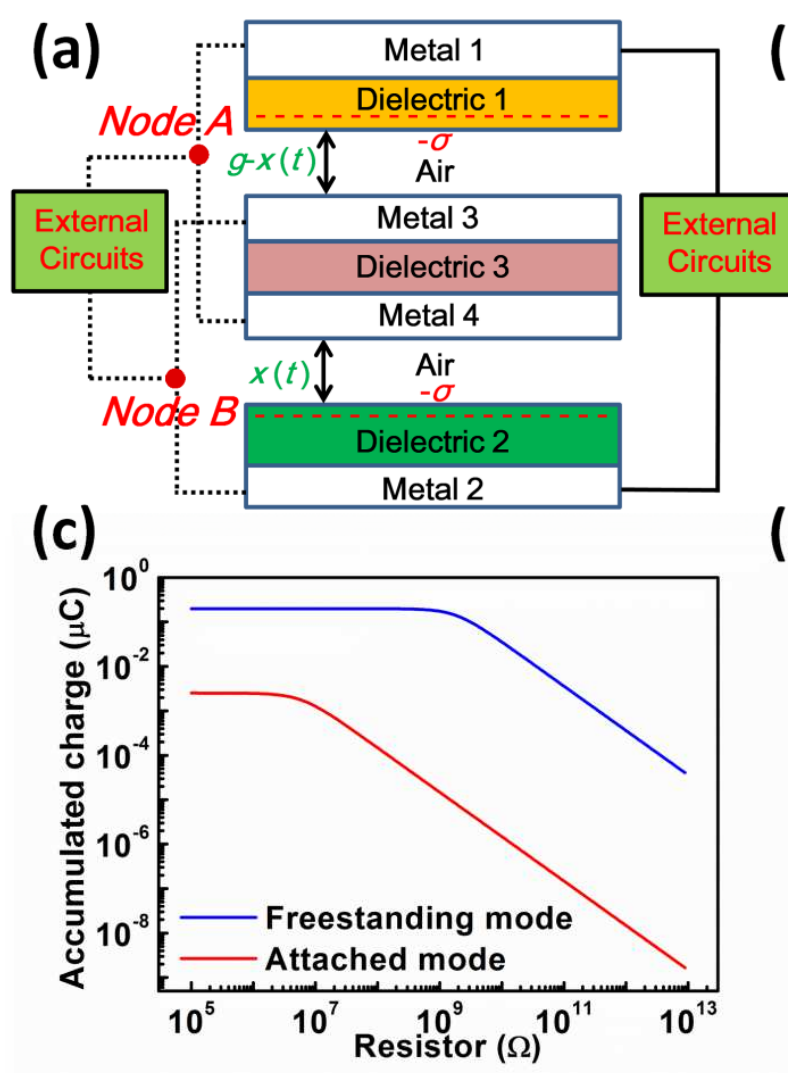

(b)

(d)
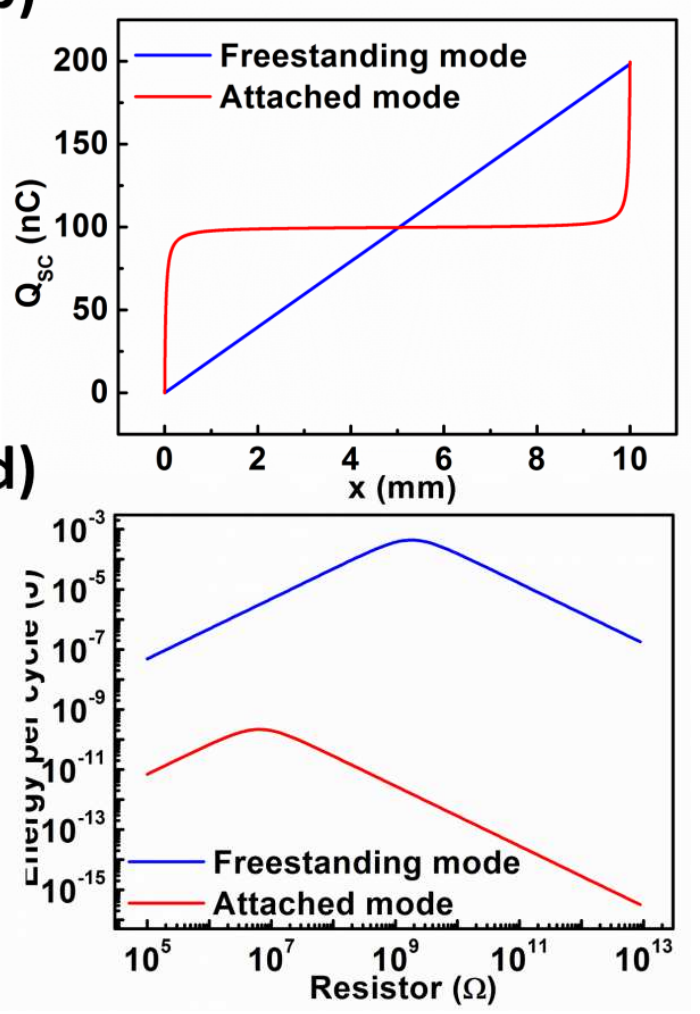

Figure 17. The advantages of contact-mode freestanding TENGs in harvesting vibration energy compared to traditional contact-mode attached-electrode TENGs. (a) Structure of the carefully designed TENG which can work in both freestanding mode and traditional attached-electrode mode. (b) Compare of short-circuit transferred charges characteristics under these two modes. (c-d) When the vibration amplitude is only a quarter of the air gap, the comparison of these two modes considering (c) the amount of the transferred charges and (d) harvested vibration energy in one cycle under different load resistance. Reproduced from reference [15]. 

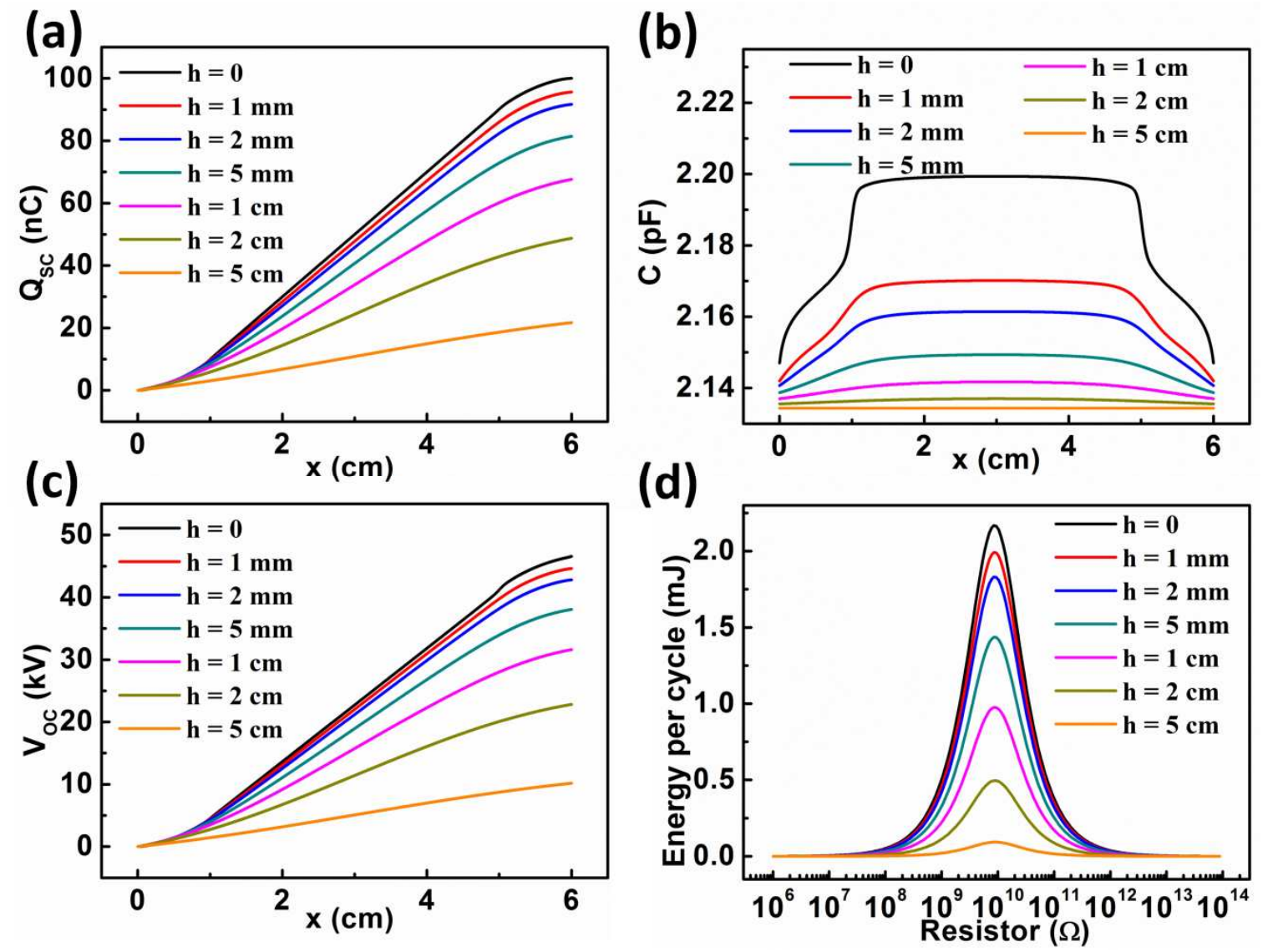

Figure 18. Tolerance of height for dielectric sliding-mode freestanding TENGs. (a-c) Influence of the freestanding height $h$ on (a) $Q_{\mathrm{SC}}$ curves under MACRS, (b) total capacitance curves, and (c) $V_{\mathrm{OC}}$ curves under MACRS of the dielectric SFTENG. (d) The harvested energy by the dielectric SFTENG in one cycle under different load resistance and different $h$. Reproduced from reference [15]. 

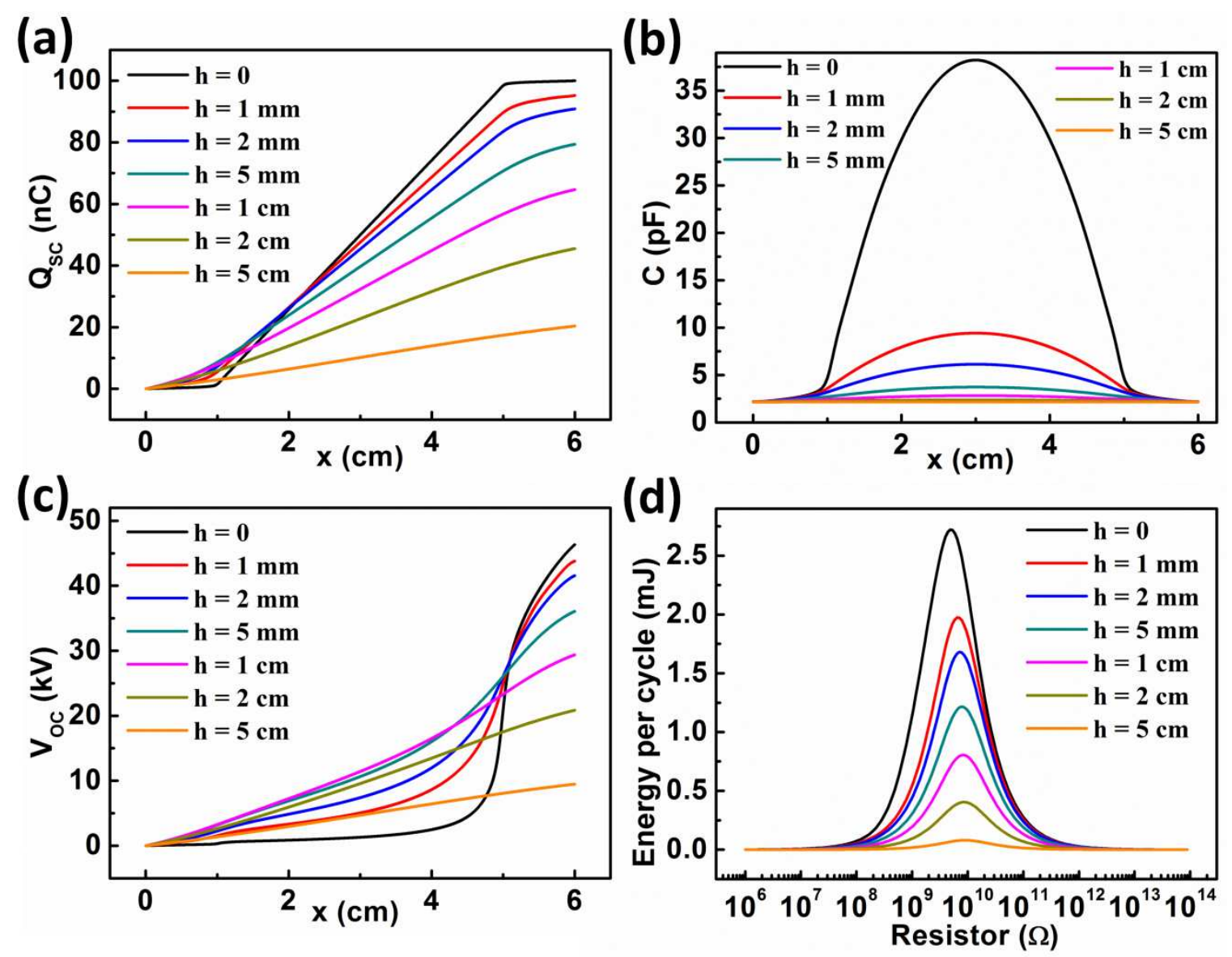

Figure 19. Tolerance of height for metal sliding-mode freestanding TENGs. (a-c) Influence of the freestanding height $h$ under $g=1 \mathrm{~cm}$ condition on (a) $Q_{\text {SC }}$ curves under MACRS, (b) total capacitance curves, and (c) $V_{\mathrm{OC}}$ curves of the metal SFTENG under MACRS. (d) The harvested energy by the metal SFTENG in one cycle under different load resistance and different $h$. Reproduced from reference [15]. 

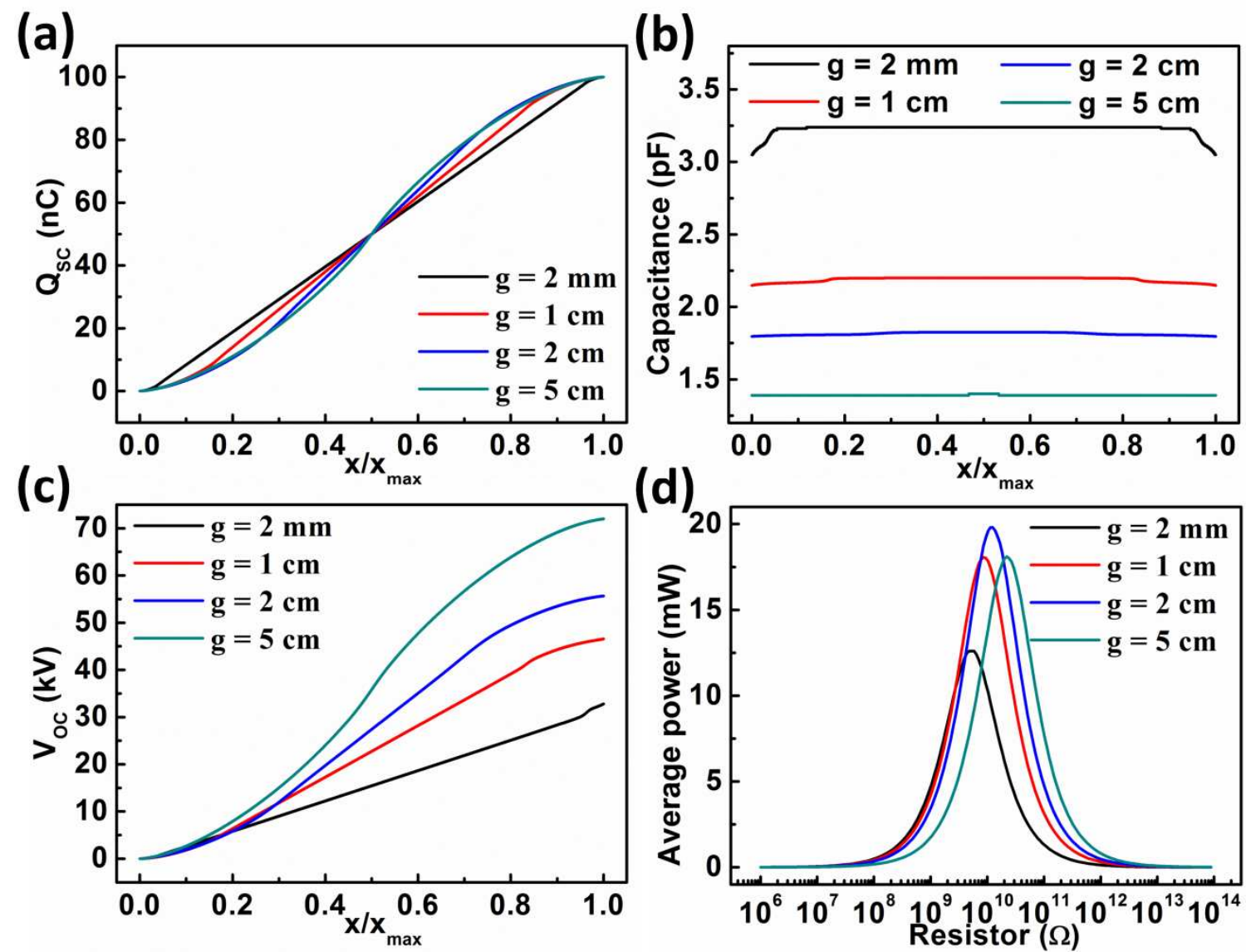

Figure 20. Influence of the gap on the output performance of dielectric sliding-mode freestanding TENGs. (a-c) Influence of the electrode gap $g$ under $h=0$ condition on (a) $Q_{\mathrm{SC}}$ curves under MACRS, (b) total capacitance curves, and (c) $V_{\mathrm{OC}}$ curves under MACRS of the dielectric SFTENG. (d) The average power by the dielectric SFTENG in one cycle under different load resistance and different $g$. Reproduced from reference [15]. 

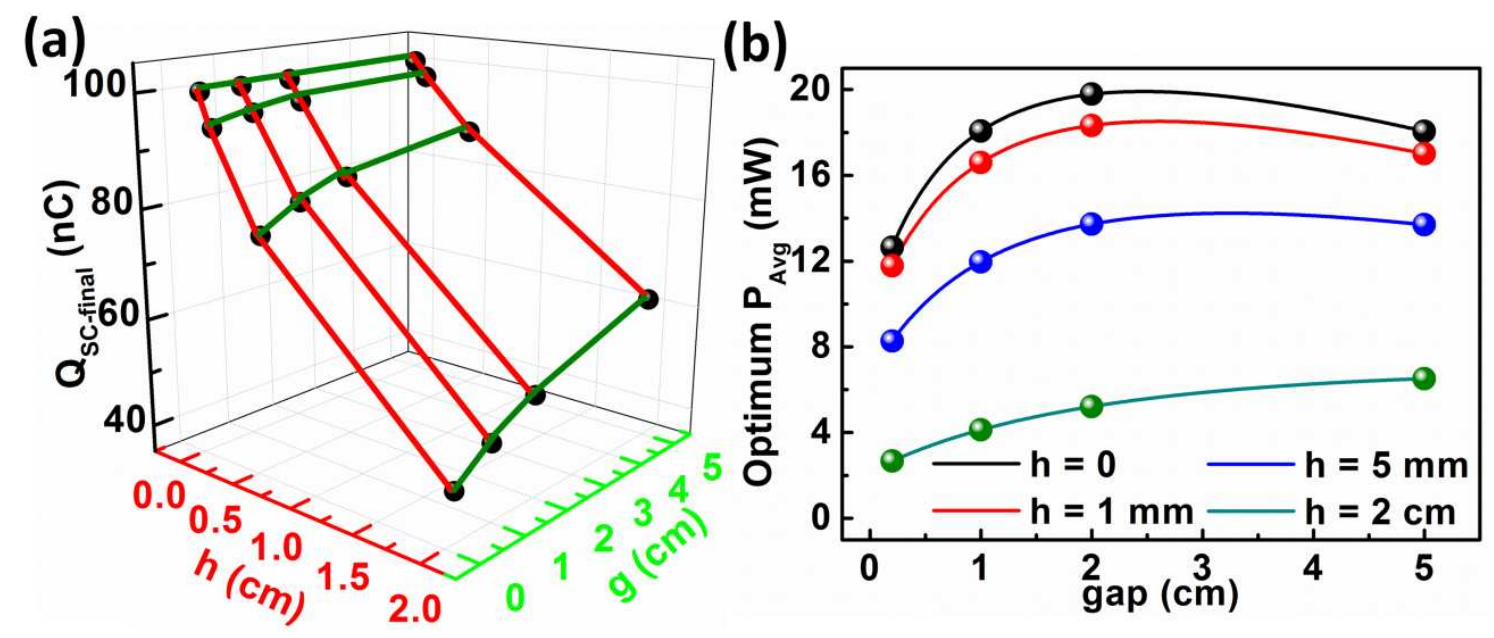

Figure 21. Coupling effect of gap and height on the output performance of dielectric sliding-mode freestanding TENGs. (a) Dependence of the short circuit transferred charge when a full separation is reached on the freestanding height and the electrode gap. (b) Influence of the freestanding height on the optimum gap at which the maximum average power is reached. Reproduced from reference [15].

\section{Tables}

Table 1. Utilized parameters in the calculation of SETENG's output characteristics

Dielectric $1 \quad \varepsilon_{\mathrm{r} 1}=2, d_{1}=100 \mu \mathrm{m}$

Thickness of electrodes $d_{m}$

$1 \mu \mathrm{m}$

Width of the structrue $w$

$5 \mathrm{~mm}$

Length of Dielectrics $l$

$5 \mathrm{~mm}$

Gap distance between electrodes $g$

$1 \mathrm{~mm}$

Tribo-charge surface density $\sigma$

Maximum separation distance $x_{\max }$

$8 \mu \mathrm{C} \mathrm{m}^{-2}$

Average Velocity $v$

$0.01 \mathrm{~m}$

TABLE 2

PARAMETERS UTILIZED IN THE CALCULATION OF OUTPUT CHARACTERISTICS FOR BOTH DIELECTRIC AND METAL SFTENGS 


\begin{tabular}{cc}
\hline \hline Structure Component & Parameter Utilized \\
\hline Dielectric & $d=500 \mu \mathrm{m}, \varepsilon_{r}=2$ \\
Width of the structure $w$ & $1 \mathrm{~m}$ \\
Length of electrode $l$ & $0.05 \mathrm{~m}$ \\
Thickness of metal electrode & $d_{\mathrm{m}}=10 \mu \mathrm{m}$ \\
and freestanding layer & \\
Tribo-charge surface density & $20 \mu \mathrm{Cm}^{-2}$ \\
$\sigma$ & $1 \mathrm{~cm}^{-1}$ \\
Electrode gap $g$ & $0 \mathrm{~mm}^{-1}$ \\
Freestanding height $h$ & $1 \mathrm{~ms}^{-1}$ \\
Motion average velocity $v$ &
\end{tabular}

Table 3. Comparison of different TENG fundamental modes

\begin{tabular}{|c|c|c|c|}
\hline & $\begin{array}{l}\text { Attached electrode } \\
\text { contact-mode and } \\
\text { sliding-mode TENGs }\end{array}$ & $\begin{array}{l}\text { Single electrode } \\
\text { TENGs }\end{array}$ & $\begin{array}{l}\text { Freestanding } \\
\text { TENGs }\end{array}$ \\
\hline $\begin{array}{l}\text { Core working } \\
\text { mechanism }\end{array}$ & \multicolumn{3}{|c|}{$\eta_{\mathrm{CT}}=\frac{1}{1+\frac{C_{1}\left(x=x_{\max }\right)}{C_{2}\left(x=x_{\max }\right)}}-\frac{1}{1+\frac{C_{1}(x=0)}{C_{2}(x=0)}}$} \\
\hline$C_{1}(x=0)$ & infinity & infinity & infinity \\
\hline$C_{2}(x=0)$ & Finite non-zero value & $\begin{array}{l}\text { Finite non-zero } \\
\text { value }\end{array}$ & $\begin{array}{l}\text { Finite non-zero } \\
\text { value }\end{array}$ \\
\hline$C_{1}\left(x=x_{\max }\right)$ & infinitesimal & infinitesimal & $\begin{array}{l}\text { Finite non-zero } \\
\text { value }\end{array}$ \\
\hline$C_{2}\left(x=x_{\max }\right)$ & Finite non-zero value & infinitesimal & infinity \\
\hline$C_{1}(x=0) / C_{2}(x=0)$ & infinity & infinity & infinity \\
\hline$C_{1}\left(x=x_{\max }\right) / C_{2}\left(x=x_{\max }\right)$ & 0 & 1 & 0 \\
\hline$\eta_{\mathrm{CT}}$ & $100 \%$ & $50 \%$ & $100 \%$ \\
\hline
\end{tabular}


Table 4. Parameters utilized in the constant velocity theoretical calculation

Dielectric 1

Dielectric 2

Area size of Dielectrics $S$

Tribo-charge surface density $\sigma$

Maximum separation distance $x_{\max }$

Average Velocity $v$
Metal, $d_{1}=0 \mu \mathrm{m}$

$d_{2}=125 \mu \mathrm{m}, \varepsilon_{\mathrm{r} 2}=3.4$

$58.0644 \mathrm{~cm}^{2}\left(9 \mathrm{inch}^{2}\right)$

$10 \mu \mathrm{Cm}^{-2}$

$0.001 \mathrm{~m}$

$0.1 \mathrm{~m} / \mathrm{s}$

TABLE 5. PARAMETERS UTILIZED IN THE CALCULATION OF THE MULTIPLE-CYCLE CHARGING BEHAVIORS FOR A CONTACT-MODE PAIRED-ELECTRODE TENG

Relative dielectric constant $\varepsilon_{\mathrm{r}} \quad 2.1$

Thickness of the dielectric $d_{\mathrm{r}} \quad 50 \mu \mathrm{m}$

Area of the dielectric $S \quad 0.005 \mathrm{~m}^{2}$

Tribo-charge surface density $\sigma \quad 40 \mu \mathrm{C} \mathrm{m}^{-2}$

Maximum separation distance $x_{\max } \quad 0.002 \mathrm{~m}$

Average Velocity $v \quad 0.1 \mathrm{~m} / \mathrm{s}$

Table 6. Parameters utilized in FEM calculations for comparing grating and plate electrodes

\begin{tabular}{cc}
\hline \hline Structure Component & Parameter Utilized \\
\hline Dielectric 1 & $\varepsilon_{r 1}=4, d_{1}=220 \mu \mathrm{m}$ \\
Dielectric 2 & $\varepsilon_{r 2}=2, d_{2}=220 \mu \mathrm{m}$ \\
Width of Dielectrics, $w$ & $0.1 \mathrm{~m}$ \\
Total Length of the top plate, $L$ & $0.16 \mathrm{~m}$ \\
Tribo-charge surface density, $\sigma$ & $7 \mu \mathrm{Cm}^{-2}$ \\
Velocity, $v$ & $1 \mathrm{~ms}^{-1}$ \\
\hline \hline
\end{tabular}

TABLE 7. PARAMETERS UTILIZED IN THE FEM CALCULATION FOR ILLUSTRATING NONIDEAL EFFECT FOR THE EQUAL-LENGTH GRATING TENGS. 


\begin{tabular}{cc}
\hline \hline Structure Component & Parameter Utilized \\
\hline Dielectric 1 & $\varepsilon_{r l}=4, d_{1}=100 \mu \mathrm{m}$ \\
Dielectric 2 & $\varepsilon_{r 2}=2, d_{2}=100 \mu \mathrm{m}$ \\
Width of Dielectrics $w$ & $1 \mathrm{~mm}$ \\
Total Length of the top plate $L$ & $1.6 \mathrm{~mm}$ \\
Tribo-charge surface density $\sigma$ & $7 \mu \mathrm{Cm}^{-2}$ \\
Velocity $v$ & $1 \mathrm{~ms}^{-1}$ \\
\hline \hline
\end{tabular}

TABLE 8

PARAMETERS FOR FEM CALCULATION FOR UNEQUAL-LENGTH GRATING TENGS

\begin{tabular}{ccc}
\hline \hline & $\begin{array}{c}\text { Dielectric-to- } \\
\text { dielectric unequal- } \\
\text { length grating } \\
\text { TENGs }\end{array}$ & $\begin{array}{c}\text { Conductor-to-dielectric } \\
\text { unequal-length grating } \\
\text { TENGs }\end{array}$ \\
\hline $\begin{array}{c}\text { Dielectric } 1 \\
\text { Dielectric } 2\end{array}$ & $\begin{array}{c}\varepsilon_{r l}=4, d_{l}=220 \mu \mathrm{m} \\
\varepsilon_{r 2}=2, d_{2}=220 \mu \mathrm{m}\end{array}$ & $\begin{array}{c}\varepsilon_{r l}=4, d_{l}=220 \mu \mathrm{m} \\
\text { Metal, } d_{2}=0 \\
0.1 \mathrm{~m}\end{array}$ \\
$\begin{array}{c}\text { Width of Dielectrics } w \\
\text { Total Length of the } \\
\text { top plate } L\end{array}$ & $0.1 \mathrm{~m}$ & $0.16 \mathrm{~m}$ \\
$\begin{array}{c}\text { Tribo-charge surface } \\
\text { density at the bottom } \\
\text { dielectric surface } \sigma \\
\text { Velocity } v\end{array}$ & $0.16 \mathrm{~m}$ & $3.5 \mu \mathrm{Cm}^{-2}$ \\
\hline \hline
\end{tabular}


Graphical abstract

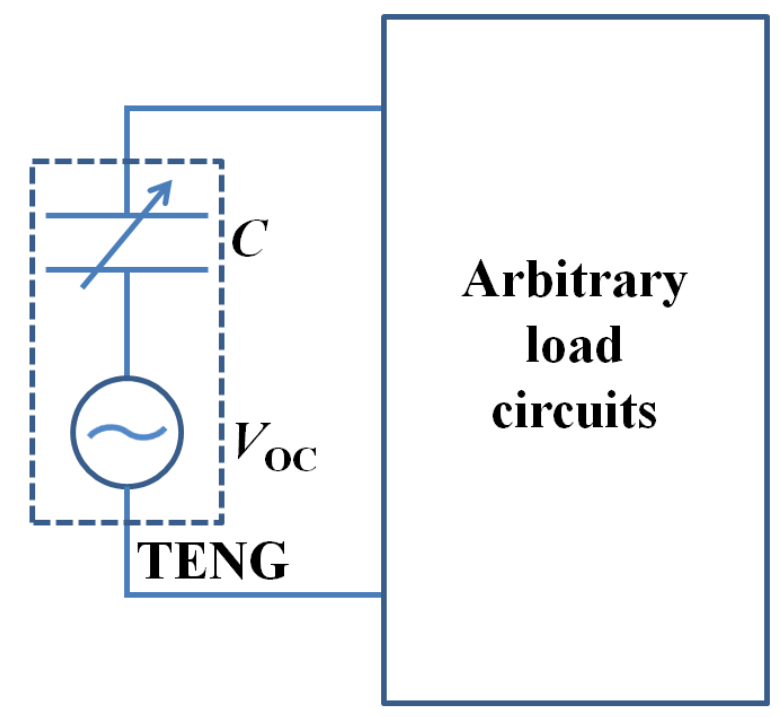

